\title{
Isomeric Tetradentate Ligand-Containing Cyclometalated Gold(III) Complexes
}

Chin-Ho Lee, Man-Chung Tang, Fred Ka-Wai Kong, Wai-Lung Cheung, Maggie Ng, Mei-Yee Chan, Vivian Wing-Wah Yam*

[*] Dr. C.-H. Lee, Dr. M.-C. Tang, Dr. F. K.-W. Kong, Mr. W.-L. Cheung, Dr. M. Ng, Dr. M.Y. Chan, Prof. V. W.-W. Yam*

Department of Chemistry, The University of Hong Kong, Pokfulam Road, Hong Kong, P. R. China. Fax: +(852) 2857-1586; Tel: +(852) 2859-2153

E-mail:wwyam@hku.hk

Supporting Information 


\section{Contents}

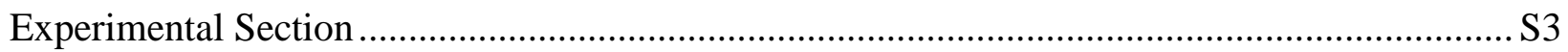

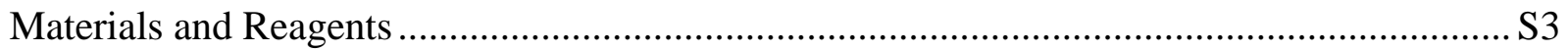

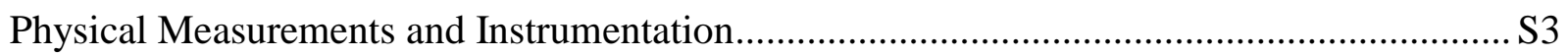

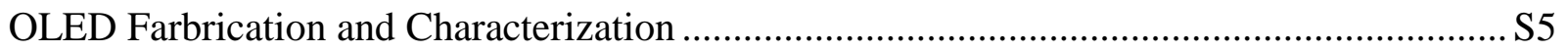

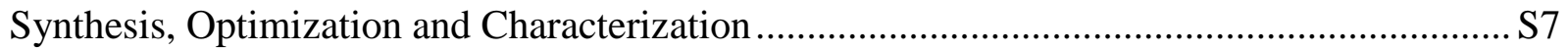

Synthesis of Cyclometalated Chlorogold(III) Complexes ................................................. S7

Synthesis of Cyclometalated Gold(III) Complexes with Tetradentate Ligands ................... S9

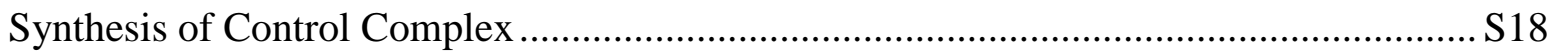

Thermogravimetric Traces............................................................................................... S19

X-Ray Crystal Structure Data and Refinement Details .................................................... S22

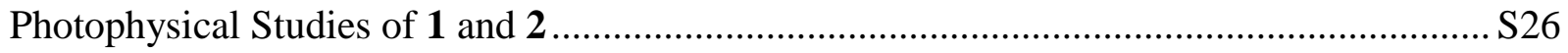

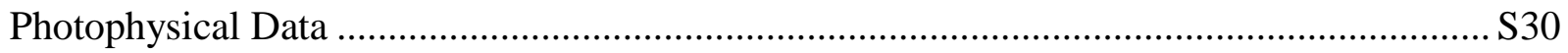

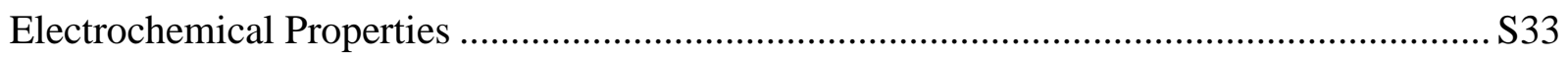

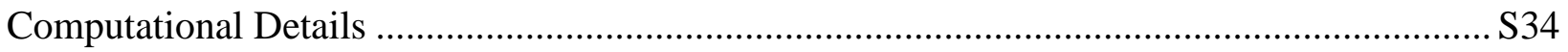

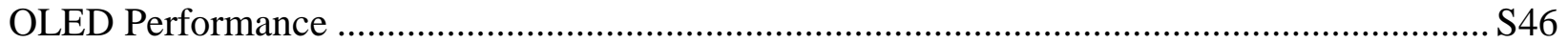

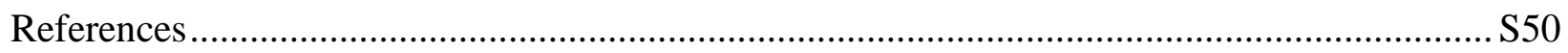




\section{Experimental Section}

\section{Materials and Reagents}

Triethylamine was distilled over calcium hydride before use. Mercury(II) acetate and 3,6-di(tertbutyl)carbazole were purchased from Aldrich Chemicals. 4-Tert-butylbenzaldehyde was purchased from TCI Chemicals. 4'-(Tert-butyl)acetophenone, 4'-fluoroacetophenone and 4'methoxyacetophenone, 7-bromo-1-tetralone, palladium(II) trifluoroacetate and palladium(II) acetylacetonate were purchased from Combi-block Chemicals. Potassium tetrachloroaurate(III), tris(4-methoxyphenyl)phosphine and tri-tert-butylphosphine were purchased from Strem Chemicals Inc. Palladium(II) acetate and palladium(II) chloride were purchased from Precious Metals Online. XPhos, SPhos and RuPhos were purchased from AK Scientific. Bis(triphenylphosphine)palladium(II) dichloride, tris(dibenzylideneacetone)dipalladium(0), tetrakis(triphenylphosphine)palladium(0), $\quad\left[1,1^{\prime}\right.$-bis(diphenylphosphine)ferrocene $]$ dichloropalladium(II) and PEPPSI-IPr catalyst were synthesized according to reported literature procedures. ${ }^{1}$ All other reagents were of analytical grade and were used as received. Cyclometalating $\mathrm{R}^{1} \mathrm{C}^{\wedge} \mathrm{N}\left({ }^{t} \mathrm{BuC}_{6} \mathrm{H}_{4}\right)^{\wedge} \mathrm{CR}^{2}$ ligands were synthesized using modified procedure of the Kröhnke pyridine synthesis. ${ }^{2}$ 3,6-Di-tert-butyl-1-(4,4,5,5-tetramethyl-1,3,2-dioxaborolan-2-yl)$9 \mathrm{H}$-carbazole was synthesized by bromination of 3,6-(di-tert-butyl)carbazole, followed by borylation, according to literature procedures. ${ }^{3}$ Tetra- $n$-butylammonium hexafluorophosphate was recrystallized for more than three times from hot absolute ethanol before use. Toluene, tetrahydrofuran and dichloromethane for reactions were purified by the Innovative Technology, Inc. PureSolv MD 5 Solvent Purification System before use. All reactions were performed under anaerobic conditions using standard Schlenk techniques under nitrogen atmosphere unless specified otherwise.

\section{Physical Measurements and Instrumentation}

UV-Visible absorption spectra were obtained by using a Cary 60 UV-visible spectrophotometer (Agilent technology) equipped with a xenon flash lamp. Transient absorption measurements were performed on a LP920-KS laser flash photolysis spectrometer (Edinburgh Instruments Ltd., Livingston, U.K.) at ambient temperature. The excitation source was the 355-nm output (third 
harmonic) of a Nd:YAG laser (Spectra-Physics Quanta-Ray Lab130 Pulsed Nd:YAG Laser), and the probe light source was a Xe900 450W xenon arc lamp. The transient absorption spectra were detected by an image intensified CCD camera (Andor) with PC plug-in controller, fully operated by L900 spectrometer software. The absorption kinetics were detected by a Hamamatsu R928 photomultiplier tube and recorded on a Tektronix model TDS3012B (100 MHz, 1.25 GS/s) digital oscilloscope and analyzed using the same software for exponential fit. ${ }^{1} \mathrm{H},{ }^{13} \mathrm{C}\left\{{ }^{1} \mathrm{H}\right\}$ and ${ }^{19} \mathrm{~F}\left\{{ }^{1} \mathrm{H}\right\}$ nuclear magnetic resonance (NMR) spectra were recorded on Bruker AVANCE 400, 500 or 600 (400 MHz, $500 \mathrm{MHz}$ and $600 \mathrm{MHz}$ for ${ }^{1} \mathrm{H}$ respectively; $100 \mathrm{MHz}, 125 \mathrm{MHz}$ and $150 \mathrm{MHz}$ for ${ }^{13} \mathrm{C}$ respectively; and $373 \mathrm{MHz}$ and $471 \mathrm{MHz}$ for ${ }^{19} \mathrm{~F}$ respectively) fourier-transform NMR spectrometers. All ${ }^{1} \mathrm{H}$ and ${ }^{13} \mathrm{C}$ chemical shift data were reported relative to tetramethylsilane, with the residual NMR solvent peak used as internal reference. ${ }^{19} \mathrm{~F}$ chemical shift data were reported with reference to $\mathrm{CF}_{3} \mathrm{COOH}(\delta-76.55 \mathrm{ppm})$. Splitting of the ${ }^{13} \mathrm{C}$ signal due to ${ }^{19} \mathrm{~F}-{ }^{13} \mathrm{C}$ coupling was not determined; instead all of them were reported as individual singlet signals. Positive electron-impact (EI) mass spectra were recorded on a Thermo Scientific DFS High Resolution Magnetic Sector Mass Spectrometer. Positive matrix assisted laser desorption ionization (MALDI) spectra were recorded on a Bruker ultraXtreme mass spectrometer using dithranol matrix. Highresolution electrospray ionization (ESI) and atmospheric pressure chemical ionization (APCI) mass spectra were recorded on a Bruker maXis II high resolution quadruple time-of-flight (QTOF) mass spectrometer. Steady-state excitation and emission spectra were recorded on a Horiba Fluoromax-4 spectrofluorometer equipped with a R928P PMT detector. Solid-state photophysical measurements were carried out with the solid sample loaded in a quartz tube inside a quartz-walled Dewar flask. Liquid nitrogen was placed into the optical Dewar flask for low temperature (77 K) photophysical measurements. The excited-state lifetimes of solution samples were measured with an Edinburgh Instruments LP980 spectrometer, while that of solid and glass samples were measured using a conventional laser system. The excitation source used was the 355-nm output (third harmonic, $8 \mathrm{~ns}$ ) of a Spectra-Physics Quanta-Ray Q-switched GCR-150 pulsed Nd:YAG laser $(10 \mathrm{~Hz})$. Luminescence decay signals were detected by a Hamamatsu R928 photomultiplier tube, recorded on a Tektronix Model TDS-620A (500 MHz, $2 \mathrm{GS} \mathrm{s}^{-1}$ ) digital oscilloscope, and analyzed by using a program for exponential fits. The excited-state lifetimes of thin film samples were measured on a Hamamatsu C11367 Quantaurus-Tau Compact Fluorescence Lifetime Spectrometer. Relative luminescence quantum yields were measured by the optical dilute method 
reported by Demas and Crosby. ${ }^{4} \mathrm{~A}$ degassed solution of $\left[\mathrm{Ru}(\mathrm{bpy})_{3}\right] \mathrm{Cl}_{2}$ in degassed acetonitrile ( $\Phi_{\text {lum }}=0.06$, excitation wavelength at $436 \mathrm{~nm}$ ) was used as the reference, ${ }^{4}$ while absolute photoluminescence quantum yields of the thin films were measured on a Hamamatsu C11347 Absolute PL Quantum Yield Measurement System. Cyclic voltammetric measurements were performed by using a CH Instruments, Inc. model CHI 620E electrochemical analyzer. All solutions for electrochemical measurements were purged with pre-purified argon gas prior to measurement. Thermogravimetric analysis (TGA) were performed with the Q50 TGA (TA instruments), in which the decomposition temperature, $\mathrm{T}_{d}$, is defined as the temperature at which the sample shows a 5\% weight loss. The crystal data were collected on beamline BL17B at the Shanghai Synchrotron Radiation Facilities (SSRF) of the Shanghai Institute of Applied Physics (SINAP). The detector used is the Rayonix MX300 CCD Detector. Data reduction and integration were performed by the HKL3000 software. ${ }^{5}$ Structure solution was solved by direct methods using $X T$ program $^{6}$ and refined by $X L$ program $^{7}$. In the final stage of the least-squares refinement, all non-hydrogen atoms were refined anisotropically. All C-bound phenyl and methylene hydrogen atoms were refined using riding model with $U_{\text {iso }}(\mathrm{H})=1.2 U_{\text {eq }}($ Carrier $)$, C-bound methyl hydrogen atoms were refined using riding model with $U_{\text {iso }}(\mathrm{H})=1.5 U_{\text {eq }}($ Carrier $)$. High performance liquid chromatography was carried out using the Waters Breeze 2 HPLC system, with In-Line Degasser AF, Pump Control Module II, two 515 HPLC pumps and a 2998 Photodiode Array Detector. The column used was a Waters SunFire prep silica column with particle size of $5 \mu \mathrm{m}$ and column dimensions of $4.6 \times 150 \mathrm{~mm}$. The column was thermostatted at $30{ }^{\circ} \mathrm{C}$ during analysis.

\section{OLED Farbrication and Characterization}

Solution-processable OLEDs with the configuration of indium-tin-oxide (ITO)/ poly(ethylenedioxythiophene):poly(styrene sulfonic acid) (PEDOT:PSS; $40 \mathrm{~nm}$ )/emissive layer (30 nm)/tris(2,4,6-trimethyl-3-(pyridine-3-yl)phenyl)borane (3TPyMB; $5 \mathrm{~nm}) / 1,3,5$-tri[(3pyridyl)-phen-3-yl]benzene (TmPyPB; $30 \mathrm{~nm}) / \mathrm{LiF}(0.8 \mathrm{~nm}) / \mathrm{Al}(100 \mathrm{~nm})$ were fabricated, in which the emissive layer was formed by mixing gold(III) complex with $N, N^{\prime}$-dicarbazolyl-3,5benzene (MCP) to prepare $1 \mathrm{~mL}$ solution in chloroform via a spin-coating technique. High-purity 3TPyMB, TmPyPB, and MCP (> 99.5 \% HPLC) were purchased from Luminescence Technology Corp. and were used as received. Current density-voltage-luminance characteristics of devices 
were simultaneously measured by a programmable Keithley 2420 source meter and a PR-655 colorimeter. All the devices were measured under ambient conditions without encapsulation. 


\section{Synthesis, Optimization and Characterization}

\section{Synthesis of Cyclometalated Chlorogold(III) Complexes}

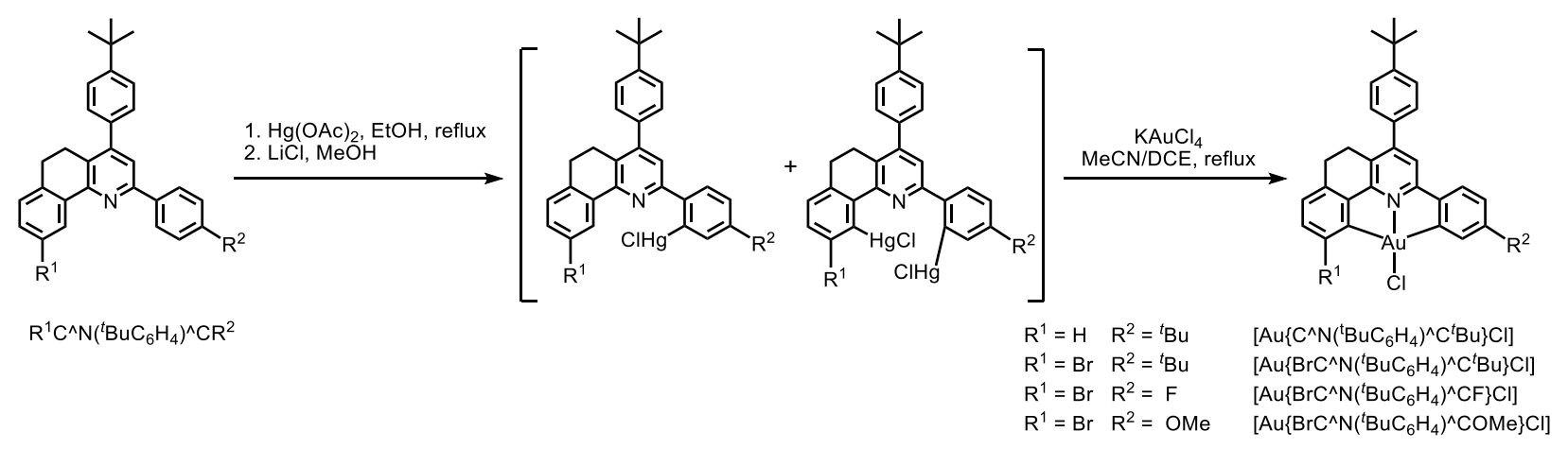

Scheme S1 Synthetic routes of cyclometalated chlorogold(III) complexes.

\section{General synthetic procedure for mercuration and auration}

To a round-bottomed flask were added $\mathrm{R}^{1} \mathrm{C}^{\wedge} \mathrm{N}\left({ }^{t} \mathrm{BuC}_{6} \mathrm{H}_{4}\right)^{\wedge} \mathrm{CR}^{2}$ (1 equiv.), $\mathrm{Hg}(\mathrm{OAc})_{2}$ (1.1 equiv.) and EtOH $\left(15 \mathrm{~mL} / \mathrm{mmol} \mathrm{R}^{1} \mathrm{C}^{\wedge} \mathrm{N}\left({ }^{t} \mathrm{BuC}_{6} \mathrm{H}_{4}\right)^{\wedge} \mathrm{CR}\right)$. The mixture was refluxed overnight. It was then cooled to room temperature, and $\mathrm{LiCl}$ (1 equiv., dissolved in $5 \mathrm{~mL} \mathrm{MeOH}$ ) was added, followed by stirring at room temperature for 30 minutes. It was then extracted with dichloromethane. The combined organic layer was washed with brine, dried over anhydrous $\mathrm{MgSO}_{4}$, filtered and concentrated to afford a mixture of mono- and dimercurated species of $\mathrm{R}^{1} \mathrm{C}^{\wedge} \mathrm{N}\left({ }^{t} \mathrm{BuC}_{6} \mathrm{H}_{4}\right)^{\wedge} \mathrm{CR}^{2}$. Then it was dissolved in 1,2-dichloroethane ( $3 \mathrm{~mL} / \mathrm{mmol}$ of the mercury species). An acetonitrile

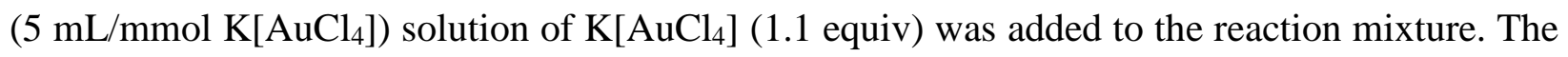
mixture was refluxed overnight. Then the yellow precipitate formed was filtered and washed with water, ice-cold acetonitrile and hexane. Then it was dried under high vacuum to afford the target product.

\section{$\left[\mathrm{Au}\left\{\mathrm{C}^{\wedge} \mathbf{N}\left({ }^{t} \mathrm{BuC}_{6} \mathrm{H}_{4}\right)^{\wedge} \mathrm{C}^{t} \mathrm{Bu}\right\} \mathrm{Cl}\right]$}

Yellow solid. Yield: $938 \mathrm{mg}, 48 \%$. ${ }^{1} \mathrm{H}$ NMR (400 MHz, dichloromethane- $\left.d_{2}, \delta / \mathrm{ppm}\right): \delta 7.85$ (d, $\left.J=2.0 \mathrm{~Hz}, 1 \mathrm{H},-\mathrm{C}_{6} \mathrm{H}_{3}-\right), 7.58\left(\mathrm{~d}, J=8.5 \mathrm{~Hz}, 2 \mathrm{H},-\mathrm{C}_{6} \mathrm{H}_{4}-\right), 7.52\left(\mathrm{~d}, J=7.4 \mathrm{~Hz}, 1 \mathrm{H},-\mathrm{C}_{6} \mathrm{H}_{3}-\right)$, 7.45-7.39 (m, 3H, $-\mathrm{C}_{6} \mathrm{H}_{4}-$ and $\left.-\mathrm{C}_{6} \mathrm{H}_{3}-\right), 7.30-7.23\left(\mathrm{~m}, 3 \mathrm{H},-\mathrm{C}_{6} \mathrm{H}_{3}-\right.$ and $\left.-\mathrm{C}_{5} \mathrm{HN}-\right), 6.94$ (dd, $J=$ $\left.7.4 \mathrm{~Hz}, 1.0 \mathrm{~Hz}, 1 \mathrm{H},-\mathrm{C}_{6} \mathrm{H}_{3}-\right), 3.02-2.92\left(\mathrm{~m}, 4 \mathrm{H},-\mathrm{CH}_{2}-\right), 1.39$ (s, 9H, $\left.{ }^{t} \mathrm{Bu}\right), 1.34\left(\mathrm{~s}, 9 \mathrm{H},-{ }^{t} \mathrm{Bu}\right)$; 
${ }^{13} \mathrm{C}\left\{{ }^{1} \mathrm{H}\right\}$ data were not available due to the low solubility of the compound; HRMS (positive ESI): Found : m/z 676.2029 [M+H] $]^{+}$. Calcd for $\mathrm{C}_{33} \mathrm{H}_{34} \mathrm{AuClN}[\mathrm{M}+\mathrm{H}]^{+}: \mathrm{m} / \mathrm{z}$ 676.2039.

\section{$\left[\mathrm{Au}\left\{\mathrm{BrC}^{\wedge} \mathrm{N}\left({ }^{t} \mathrm{BuC}_{6} \mathrm{H}_{4}\right)^{\wedge} \mathrm{C}^{t} \mathrm{Bu}\right\} \mathrm{Cl}\right]$}

Yellow solid. Yield: $446 \mathrm{mg}, 44 \% .{ }^{1} \mathrm{H}$ NMR (500 MHz, dichloromethane- $\left.d_{2}, \delta / \mathrm{ppm}\right): \delta 7.91$ (d, $\left.J=2.0 \mathrm{~Hz}, 1 \mathrm{H},-\mathrm{C}_{6} \mathrm{H}_{3}-\right), 7.58\left(\mathrm{~d}, J=8.3 \mathrm{~Hz}, 2 \mathrm{H},-\mathrm{C}_{6} \mathrm{H}_{4}-\right), 7.45\left(\mathrm{~d}, J=8.3 \mathrm{~Hz}, 2 \mathrm{H},-\mathrm{C}_{6} \mathrm{H}_{4}-\right), 7.34$ $\left(\mathrm{d}, J=8.0 \mathrm{~Hz}, 1 \mathrm{H},-\mathrm{C}_{6} \mathrm{H}_{2}-\right), 7.32\left(\mathrm{~d}, J=8.0 \mathrm{~Hz}, 1 \mathrm{H},-\mathrm{C}_{6} \mathrm{H}_{2}-\right), 7.25(\mathrm{dd}, J=8.1 \mathrm{~Hz}, 2.0 \mathrm{~Hz}, 1 \mathrm{H}$, $\left.-\mathrm{C}_{6} \mathrm{H}_{3}-\right), 7.23\left(\mathrm{~s}, 1 \mathrm{H},-\mathrm{C}_{5} \mathrm{HN}-\right), 6.72\left(\mathrm{~d}, J=8.1 \mathrm{~Hz}, 1 \mathrm{H},-\mathrm{C}_{6} \mathrm{H}_{3}-\right)$, 3.03-2.99 (m, 2H, $\left.-\mathrm{CH}_{2}-\right)$, 2.93-2.88 (m, 2H, $\left.-\mathrm{CH}_{2}-\right), 1.39\left(\mathrm{~s}, 9 \mathrm{H},-{ }^{t} \mathrm{Bu}\right), 1.32\left(\mathrm{~s}, 9 \mathrm{H},-{ }^{t} \mathrm{Bu}\right) ;{ }^{13} \mathrm{C}\left\{{ }^{1} \mathrm{H}\right\}$ data were not available due to the low solubility of the compound; HRMS (positive APCI): Found : m/z 754.1082 [M+H] ${ }^{+}$. Calcd for $\mathrm{C}_{33} \mathrm{H}_{33} \mathrm{AuBrClN}[\mathrm{M}+\mathrm{H}]^{+}: \mathrm{m} / \mathrm{z} 754.1145$.

\section{$\left[\mathrm{Au}\left\{\mathrm{BrC}^{\wedge} \mathrm{N}\left({ }^{t} \mathrm{BuC}_{6} \mathrm{H}_{4}\right)^{\wedge} \mathrm{CF}\right\} \mathrm{Cl}\right]$}

Yellow solid. Yield: $942 \mathrm{mg}, 58 \%$. ${ }^{1} \mathrm{H}$ NMR (500 MHz, dichloromethane- $\left.d_{2}, \delta / \mathrm{ppm}\right): \delta 7.74$ (dd, $\left.J=8.2 \mathrm{~Hz}, 2.7 \mathrm{~Hz}, 1 \mathrm{H},-\mathrm{C}_{6} \mathrm{H}_{3}-\right), 7.57\left(\mathrm{~d}, J=8.2 \mathrm{~Hz}, 2 \mathrm{H},-\mathrm{C}_{6} \mathrm{H}_{4}-\right), 7.50-7.43\left(\mathrm{~m}, 3 \mathrm{H},-\mathrm{C}_{6} \mathrm{H}_{2}-\right.$ and $\left.-\mathrm{C}_{5} \mathrm{HN}-\right), 7.37\left(\mathrm{~d}, J=8.2 \mathrm{~Hz}, 2 \mathrm{H},-\mathrm{C}_{6} \mathrm{H}_{4}-\right), 7.05\left(\mathrm{~s}, 1 \mathrm{H},-\mathrm{C}_{6} \mathrm{H}_{3}-\right), 6.80(\mathrm{~d}, J=8.2 \mathrm{~Hz}, 1 \mathrm{H}$, $\left.-\mathrm{C}_{6} \mathrm{H}_{3}-\right)$, 3.02-2.97 (m, 2H, $\left.-\mathrm{CH}_{2}-\right)$, 2.93-2.87 (m, 2H, $\left.-\mathrm{CH}_{2}-\right), 1.40\left(\mathrm{~s}, 9 \mathrm{H},-{ }^{t} \mathrm{Bu}\right) ;{ }^{13} \mathrm{C}\left\{{ }^{1} \mathrm{H}\right\}$ and

${ }^{19} \mathrm{~F}\left\{{ }^{1} \mathrm{H}\right\}$ data were not available due to the low solubility of the compound; HRMS (positive APCI): Found : m/z 716.0371 [M+H] $]^{+}$. Calcd for $\mathrm{C}_{29} \mathrm{H}_{24} \mathrm{AuBrClFN}[\mathrm{M}+\mathrm{H}]^{+}: \mathrm{m} / \mathrm{z} 716.0425$.

\section{$\left[\mathrm{Au}\left\{\mathrm{BrC}^{\wedge} \mathrm{N}\left({ }^{t} \mathrm{BuC}_{6} \mathrm{H}_{4}\right)^{\wedge} \mathrm{COMe}\right\} \mathrm{Cl}\right]$}

Yellow solid. Yield: $502 \mathrm{mg}, 36 \% .{ }^{1} \mathrm{H}$ NMR (400 MHz, dichloromethane- $\left.d_{2}, \delta / \mathrm{ppm}\right): \delta 7.57$ (d, $\left.J=7.8 \mathrm{~Hz}, 2 \mathrm{H},-\mathrm{C}_{6} \mathrm{H}_{4}-\right), 7.50\left(\mathrm{~d}, J=2.4 \mathrm{~Hz}, 1 \mathrm{H},-\mathrm{C}_{6} \mathrm{H}_{3}-\right), 7.47-7.43\left(\mathrm{~m}, 2 \mathrm{H},-\mathrm{C}_{6} \mathrm{H}_{2}-\right.$ and $\left.-\mathrm{C}_{6} \mathrm{H}_{3}-\right), 7.39$ (d, J=7.8 Hz, 2H, $\left.-\mathrm{C}_{6} \mathrm{H}_{4}-\right), 7.24\left(\mathrm{~s}, 1 \mathrm{H},-\mathrm{C}_{5} \mathrm{HN}-\right), 6.84\left(\mathrm{~d}, J=7.5 \mathrm{~Hz}, 1 \mathrm{H},-\mathrm{C}_{6} \mathrm{H}_{2}-\right)$, $6.76\left(\mathrm{~d}, J=8.5 \mathrm{~Hz}, 1 \mathrm{H},-\mathrm{C}_{6} \mathrm{H}_{3}-\right), 3.88(\mathrm{~s}, 3 \mathrm{H},-\mathrm{OMe}), 2.99-2.93\left(\mathrm{~m}, 2 \mathrm{H},-\mathrm{CH}_{2}-\right), 2.93-2.88(\mathrm{~m}$, $\left.2 \mathrm{H},-\mathrm{CH}_{2}-\right), 1.38\left(\mathrm{~s}, 9 \mathrm{H},-{ }^{t} \mathrm{Bu}\right) ;{ }^{13} \mathrm{C}\left\{{ }^{1} \mathrm{H}\right\}$ data were not available due to the low solubility of the compound; HRMS (positive APCI): Found : m/z $728.0559[\mathrm{M}+\mathrm{H}]^{+}$. Calcd for $\mathrm{C}_{30} \mathrm{H}_{27} \mathrm{AuBrClNO}$ $[\mathrm{M}+\mathrm{H}]^{+}: \mathrm{m} / \mathrm{z} 728.0625$. 


\section{Synthesis of Cyclometalated Gold(III) Complexes with Tetradentate Ligands}

\section{Initial attempt for the synthesis of tetradentate ligand-containing gold(III) complexes}

To a two-necked flask were added $\left[\mathrm{Au}\left\{\mathrm{BrC}^{\wedge} \mathrm{N}\left({ }^{t} \mathrm{BuC}_{6} \mathrm{H}_{4}\right)^{\wedge} \mathrm{C}^{t} \mathrm{Bu}\right\} \mathrm{Cl}\right](280 \mathrm{mg}, 0.37 \mathrm{mmol}), 3,6$-ditert-butyl-1-(4,4,5,5-tetramethyl-1,3,2-dioxaborolan-2-yl)-9H-carbazole (157 mg, $0.38 \mathrm{mmol})$, $\mathrm{Pd}\left(\mathrm{PPh}_{3}\right)_{2} \mathrm{Cl}_{2}(26 \mathrm{mg}, 0.04 \mathrm{mmol})$ and $\mathrm{K}_{2} \mathrm{CO}_{3}(191 \mathrm{mg}, 1.38 \mathrm{mmol})$. The mixture was degassed and then degassed tetrahydrofuran $(15 \mathrm{~mL})$ and water $(3 \mathrm{~mL})$ were added to the reaction mixture. The mixture was heated at $90{ }^{\circ} \mathrm{C}$ overnight. Then it was concentrated and purified by column chromatography. The product was further purified by recrystallization from its ethyl acetate solution to give $1(12 \%)$ as an orange solid. The mother liquor was left on the bench overnight and yellow solid was found to precipitate out of the solution, which is $\mathbf{2}(6 \%)$.

\section{$\left[\mathrm{Au}\left\{N-{ }^{t} \mathrm{BuCbz}-\mathrm{C}^{\wedge} \mathrm{N}\left({ }^{t} \mathrm{BuC}_{6} \mathrm{H}_{4}\right)^{\wedge} \mathrm{C}^{t} \mathrm{Bu}\right\}\right](\mathbf{1})$}

Orange solid. Yield: $40 \mathrm{mg}, 12 \%$. ${ }^{1} \mathrm{H}$ NMR (500 MHz, dichloromethane- $d_{2}, \delta / \mathrm{ppm}$ ): $\delta 8.24-8.18$ (m, $4 \mathrm{H},-\mathrm{C}_{6} \mathrm{H}_{2}-$ and $-\mathrm{C}_{6} \mathrm{H}_{3}-$ of $\mathrm{C}^{\wedge} \mathrm{N}^{\wedge} \mathrm{C}$ and $\left.-\mathrm{Cbz}^{-}\right), 8.11(\mathrm{~d}, J=1.9 \mathrm{~Hz}, 1 \mathrm{H},-\mathrm{Cbz}-), 7.88(\mathrm{~d}, J=$ $8.5 \mathrm{~Hz}, 1 \mathrm{H},-\mathrm{Cbz}-), 7.45\left(\mathrm{~d}, J=8.0 \mathrm{~Hz}, 2 \mathrm{H},-\mathrm{C}_{6} \mathrm{H}_{4}{ }^{t} \mathrm{Bu}\right.$ of $\left.\mathrm{C}^{\wedge} \mathrm{N}^{\wedge} \mathrm{C}\right), 7.40\left(\mathrm{~d}, J=8.0 \mathrm{~Hz}, 1 \mathrm{H},-\mathrm{C}_{6} \mathrm{H}_{3}-\right.$ of $\left.\mathrm{C}^{\wedge} \mathrm{N}^{\wedge} \mathrm{C}\right), 7.36(\mathrm{dd}, J=8.5 \mathrm{~Hz}, 2.0 \mathrm{~Hz}, 1 \mathrm{H},-\mathrm{Cbz}-), 7.26\left(\mathrm{dd}, J=8.0 \mathrm{~Hz}, 2.0 \mathrm{~Hz}, 1 \mathrm{H},-\mathrm{C}_{6} \mathrm{H}_{3}-\right.$ of $\left.\mathrm{C}^{\wedge} \mathrm{N}^{\wedge} \mathrm{C}\right), 7.19\left(\mathrm{~d}, J=8.0 \mathrm{~Hz}, 2 \mathrm{H},-\mathrm{C}_{6} \mathrm{H}_{4}{ }^{t} \mathrm{Bu}\right.$ of $\left.\mathrm{C}^{\wedge} \mathrm{N}^{\wedge} \mathrm{C}\right), 7.12\left(\mathrm{~d}, J=8.0 \mathrm{~Hz}, 1 \mathrm{H},-\mathrm{C}_{6} \mathrm{H}_{2}-\right.$ of $\left.\mathrm{C}^{\wedge} \mathrm{N}^{\wedge} \mathrm{C}\right), 7.02\left(\mathrm{~s}, 1 \mathrm{H},-\mathrm{C}_{5} \mathrm{NH}-\right.$ of $\left.\mathrm{C}^{\wedge} \mathrm{N}^{\wedge} \mathrm{C}\right), 2.80\left(\mathrm{t}, J=7.6 \mathrm{~Hz}, 2 \mathrm{H},-\mathrm{CH}_{2}-\right.$ of $\left.\mathrm{C}^{\wedge} \mathrm{N}^{\wedge} \mathrm{C}\right), 2.63(\mathrm{t}, J$ $=7.7 \mathrm{~Hz}, 2 \mathrm{H},-\mathrm{CH}_{2}-$ of $\left.\mathrm{C}^{\wedge} \mathrm{N}^{\wedge} \mathrm{C}\right), 1.59\left(\mathrm{~s}, 9 \mathrm{H},-{ }^{t} \mathrm{Bu}\right.$ of $\left.-\mathrm{Cbz}-\right), 1.47\left(\mathrm{~s}, 9 \mathrm{H},{ }^{t} \mathrm{Bu}\right.$ of $\left.-\mathrm{Cbz}-\right), 1.39$ (s, 9H, $-{ }^{t} \mathrm{Bu}$ of $\left.-\mathrm{C}_{6} \mathrm{H}_{4}{ }^{t} \mathrm{Bu}\right), 1.23$ (s, 9H, $-{ }^{t} \mathrm{Bu}$ of $\left.-\mathrm{C}_{6} \mathrm{H}_{3}{ }^{t} \mathrm{Bu}\right) ;{ }^{13} \mathrm{C}\left\{{ }^{1} \mathrm{H}\right\}$ NMR (125 MHz, dichloromethane- $\left.d_{2}, \delta / \mathrm{ppm}\right): \delta 169.90,161.78,160.29,160.04,153.62,153.24,152.63,148.27$, 145.53, 145.20, 141.98, 140.71, 138.22, 137.48, 135.69, 134.76, 131.47, 128.81, 127.50, 127.30, $126.47,126.43,126.11,125.83,125.31,124.89,123.50,122.73,118.54,118.48,117.44,116.21$, 115.64, 35.77, 35.26, 35.20, 35.01, 32.62, 32.39, 31.64, 31.45, 27.37, 24.85; HRMS (positive APCI): Found : $\mathrm{m} / \mathrm{z} 917.4032[\mathrm{M}+\mathrm{H}]^{+}$. Calcd for $\mathrm{C}_{53} \mathrm{H}_{56} \mathrm{AuN}_{2}[\mathrm{M}+\mathrm{H}]^{+}: \mathrm{m} / \mathrm{z} 917.4104$. 


\section{$\left[\mathrm{Au}\left\{C-{ }^{t} \mathrm{BuCbz}^{-}-\mathrm{C}^{\wedge} \mathrm{N}\left({ }^{t} \mathrm{BuC}_{6} \mathrm{H}_{4}\right)^{\wedge} \mathrm{C}^{t} \mathrm{Bu}\right\}\right](2)$}

Yellow solid. Yield: $20 \mathrm{mg}, 6 \%$. ${ }^{1} \mathrm{H}$ NMR (500 MHz, dichloromethane- $\left.d_{2}, \delta / \mathrm{ppm}\right): \delta 8.65$ (d, $J=$ $1.9 \mathrm{~Hz}, 1 \mathrm{H},-\mathrm{Cbz}-), 8.35\left(\mathrm{~d}, J=2.0 \mathrm{~Hz}, 1 \mathrm{H},-\mathrm{C}_{6} \mathrm{H}_{3}-\right.$ of $\left.\mathrm{C}^{\wedge} \mathrm{N}^{\wedge} \mathrm{C}\right), 8.25\left(\mathrm{~d}, J=8.4 \mathrm{~Hz}, 1 \mathrm{H},-\mathrm{C}_{6} \mathrm{H}_{2}-\right.$ of $\left.\mathrm{C}^{\wedge} \mathrm{N}^{\wedge} \mathrm{C}\right), 8.20(\mathrm{~d}, J=1.9 \mathrm{~Hz}, 1 \mathrm{H},-\mathrm{Cbz}-), 8.18\left(\mathrm{~d}, J=2.1 \mathrm{~Hz}, 1 \mathrm{H},-\mathrm{Cbz}^{-}\right), 8.11(\mathrm{~d}, J=8.8 \mathrm{~Hz}$, $\left.1 \mathrm{H},-\mathrm{Cbz}_{-}\right), 7.62\left(\mathrm{~d}, J=8.1 \mathrm{~Hz}, 1 \mathrm{H},-\mathrm{C}_{6} \mathrm{H}_{3}-\right.$ of $\left.\mathrm{C}^{\wedge} \mathrm{N}^{\wedge} \mathrm{C}\right), 7.55\left(\mathrm{dd}, J=8.8 \mathrm{~Hz}, 2.1 \mathrm{~Hz}, 1 \mathrm{H},-\mathrm{Cbz}^{-}\right)$,

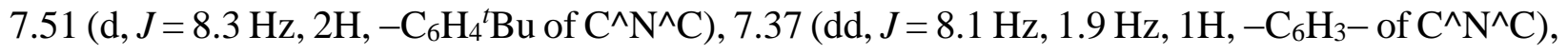
$7.27\left(\mathrm{~d}, J=8.3 \mathrm{~Hz}, 2 \mathrm{H},-\mathrm{C}_{6} \mathrm{H}_{4}{ }^{t} \mathrm{Bu}\right.$ of $\left.\mathrm{C}^{\wedge} \mathrm{N}^{\wedge} \mathrm{C}\right), 7.24\left(\mathrm{~s}, 1 \mathrm{H},-\mathrm{C}_{5} \mathrm{NH}-\right.$ of $\left.\mathrm{C}^{\wedge} \mathrm{N}^{\wedge} \mathrm{C}\right), 7.10(\mathrm{~d}, J=8.4$ $\mathrm{Hz}, 1 \mathrm{H},-\mathrm{C}_{6} \mathrm{H}_{2}-$ of $\left.\mathrm{C}^{\wedge} \mathrm{N}^{\wedge} \mathrm{C}\right), 2.97-2.92\left(\mathrm{~m}, 2 \mathrm{H},-\mathrm{CH}_{2}-\right.$ of $\left.\mathrm{C}^{\wedge} \mathrm{N}^{\wedge} \mathrm{C}\right), 2.87-2.83\left(\mathrm{~m}, 2 \mathrm{H},-\mathrm{CH}_{2}-\right.$ of $\left.\mathrm{C}^{\wedge} \mathrm{N}^{\wedge} \mathrm{C}\right), 1.65\left(\mathrm{~s}, 9 \mathrm{H},-{ }^{t} \mathrm{Bu}\right.$ of $\left.-\mathrm{Cbz}-\right), 1.49\left(\mathrm{~s}, 9 \mathrm{H},-^{t} \mathrm{Bu}\right.$ of $\left.-\mathrm{Cbz}-\right), 1.48\left(\mathrm{~s}, 9 \mathrm{H},{ }^{t} \mathrm{Bu}\right.$ of $\left.-\mathrm{C}_{6} \mathrm{H}_{3}{ }^{t} \mathrm{Bu}\right)$, $1.40\left(\mathrm{~s}, 9 \mathrm{H},-{ }^{t} \mathrm{Bu}\right.$ of $\left.-\mathrm{C}_{6} \mathrm{H}_{4}{ }^{t} \mathrm{Bu}\right) ;{ }^{13} \mathrm{C}\left\{{ }^{1} \mathrm{H}\right\}$ NMR $\left(125 \mathrm{MHz}\right.$, dichloromethane- $\left.d_{2}, \delta / \mathrm{ppm}\right): \delta 170.82$, 170.23, 160.08, 153.90, 153.70, 152.69, 148.31, 147.46, 145.35, 144.13, 143.72, 142.76, 137.46, 136.27, 135.53, 135.43, 133.39, 133.26, 130.87, 128.84, 128.09, 127.85, 127.41, 126.11, 125.72, $123.79,123.69,118.35,118.13,116.95,115.67,115.31,114.27,35.92,35.25,35.14,34.77,32.85$, 32.19, 31.98, 31.62, 27.64, 25.63; HRMS (positive APCI): Found: m/z $917.4049[\mathrm{M}+\mathrm{H}]^{+}$. Calcd for $\mathrm{C}_{53} \mathrm{H}_{56} \mathrm{AuN}_{2}[\mathrm{M}+\mathrm{H}]^{+}: \mathrm{m} / \mathrm{z} 917.4109$. 

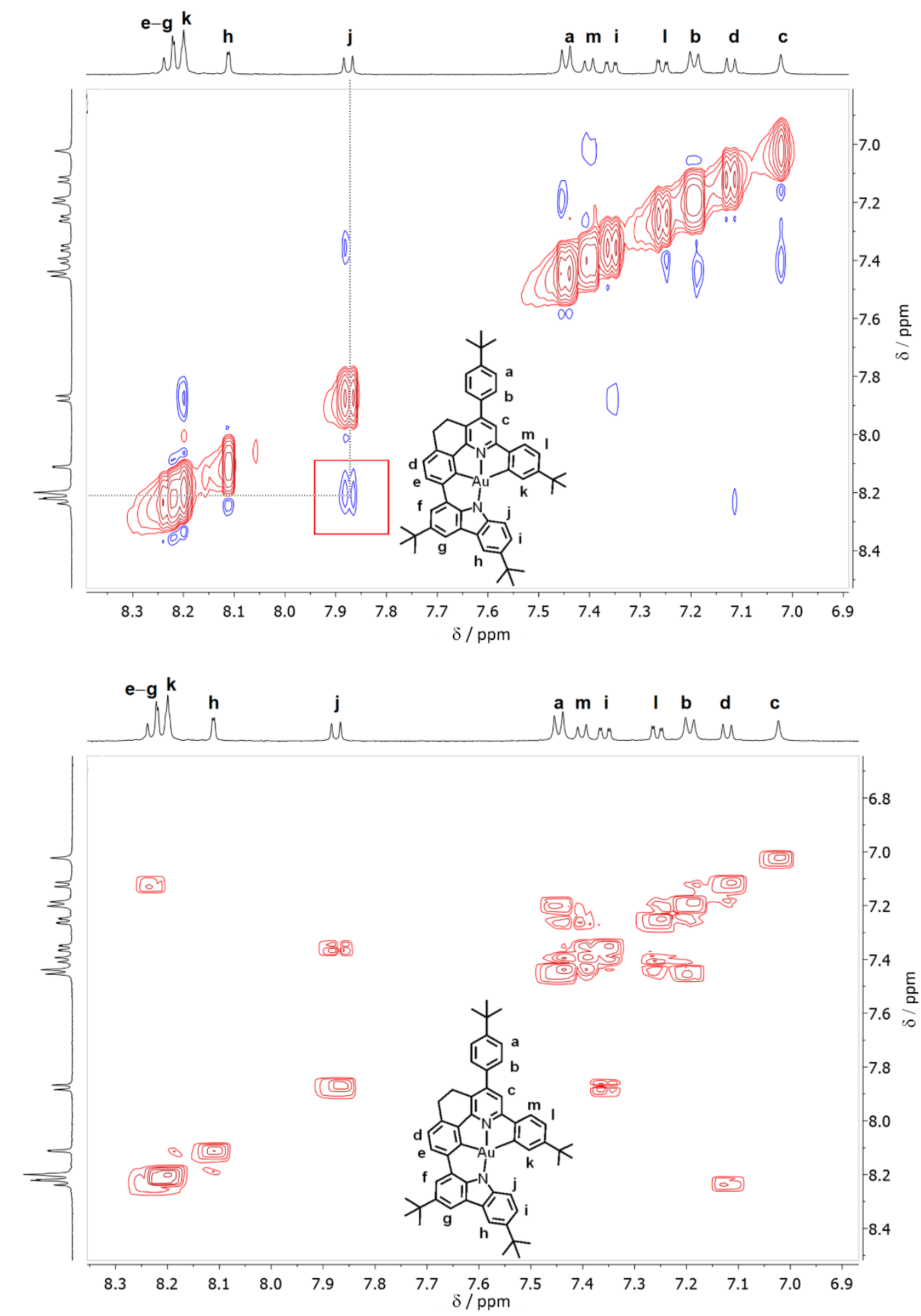

Figure S1 Partial ${ }^{1} \mathrm{H}-{ }^{1} \mathrm{H}$ NOESY (top) and COSY (bottom) NMR spectra of $\mathbf{1}$ in dichloromethane- $d_{2}$ at $298 \mathrm{~K}$. 

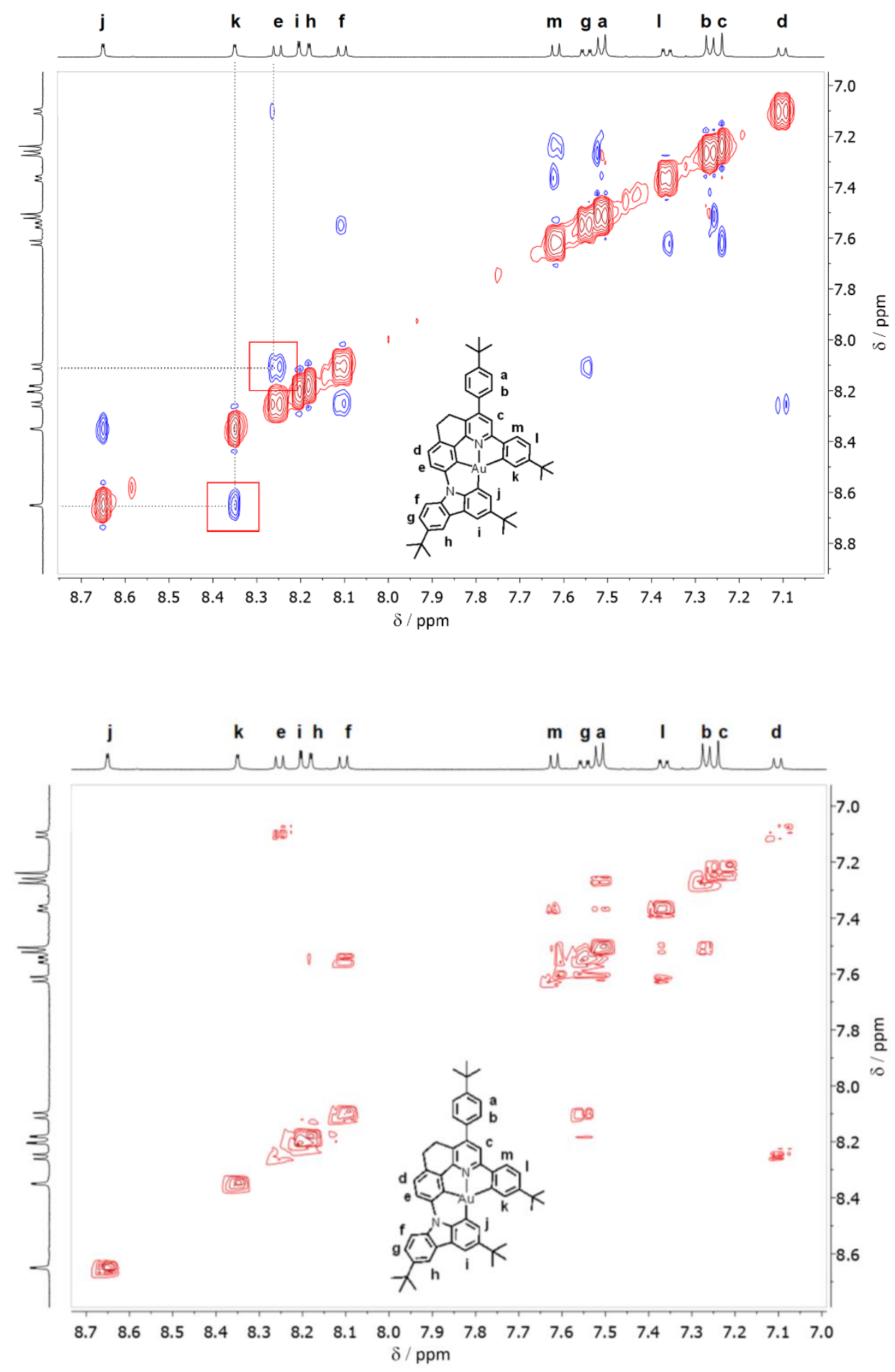

Figure S2 Partial ${ }^{1} \mathrm{H}-{ }^{1} \mathrm{H}$ NOESY (top) and COSY (bottom) NMR spectra of 2 in dichloromethane- $d_{2}$ at $298 \mathrm{~K}$. 


\section{Reaction optimization for synthesizing tetradentate ligand-containing gold(III) complexes using HPLC}

Similar experimental procedures were applied as in the previous section, except that the crude reaction mixture was subjected to solvent extraction using dichloromethane. Then the solution was filtered through a short silica gel column using dichloromethane as eluent. Upon removal of solvent, the crude mixture was re-dissolved in HPLC grade dichloromethane and filtered through $0.45 \mu \mathrm{m}$ PTFE filter before HPLC analysis. The gradient condition was as follows.

Table S1 Gradient condition for HPLC run.

\begin{tabular}{cccc}
\hline Time / min & $\begin{array}{c}\text { Flow rate / } \\
\text { mL min }^{-1}\end{array}$ & \multicolumn{2}{c}{ Mobile phase / \% } \\
\cline { 3 - 4 } & 1.5 & 100 & Dichloromethane \\
\hline 0 & 1.5 & 93 & 0 \\
1 & 1.5 & 83 & 7 \\
16 & 1.5 & 83 & 17 \\
35 & 2.0 & 0 & 100 \\
36 & 2.0 & 0 & 100 \\
46 & 2.0 & 100 & 0 \\
47 & 2.0 & 100 & 0 \\
57 & 1.5 & 100 & 0 \\
58 & 1.5 & 100 & 0 \\
68 & & &
\end{tabular}

${ }^{[a]}$ Half water-saturated hexane. 
Table S2 Reaction optimization studies by HPLC.
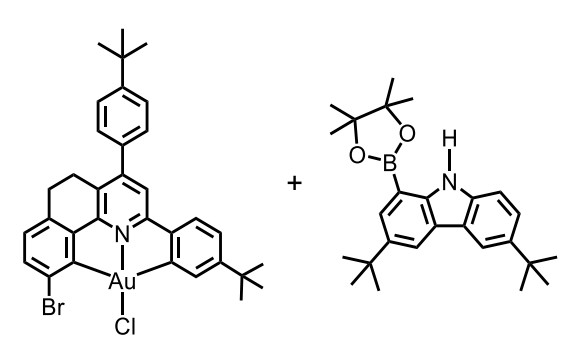

[Pd], base, additive solvent

$90^{\circ} \mathrm{C}$

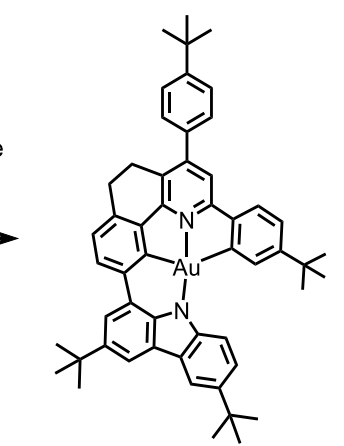

$\left[\mathrm{Au}\left\{\mathrm{N}-{ }^{t} \mathrm{BuCbz}-\mathrm{C}^{\wedge} \mathrm{N}\left(\mathrm{BBuC}_{6} \mathrm{H}_{4}\right)^{\wedge} \mathrm{C}^{t} \mathrm{Bu}\right\}\right]$ (1)

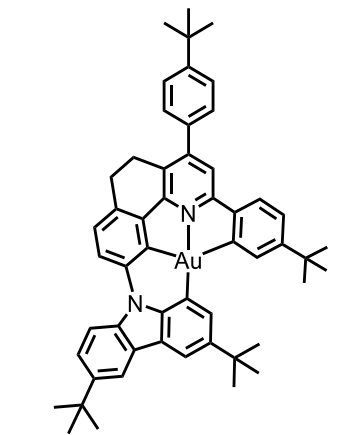

$\operatorname{Au}\left\{\mathrm{BrC}^{\wedge} N\left({ }^{t} \mathrm{BuC}_{6} \mathrm{H}_{4}\right)^{\wedge} \mathrm{C}^{t} \mathrm{Bu}\right\} \mathrm{C}$

$\left[\mathrm{Au}\left\{\mathrm{C}-{ }^{t} \mathrm{BuCbz}-\mathrm{C}^{\wedge} \mathrm{N}\left({ }^{t} \mathrm{BuC}_{6} \mathrm{H}_{4}\right)^{\wedge} \mathrm{C}^{t} \mathrm{Bu}\right\}\right]$ (2)

\begin{tabular}{|c|c|c|c|c|c|c|}
\hline Trial & [Pd] (0.1 equiv.) & Base (3.7 equiv.) & Additive (x equiv.) & Solvent & Yield of $1 / \%^{[\mathrm{a}]}$ & Yield of $2 / \%{ }^{[a]}$ \\
\hline 1 & $\mathrm{Pd}\left(\mathrm{PPh}_{3}\right)_{2} \mathrm{Cl}_{2}$ & $\mathrm{~K}_{2} \mathrm{CO}_{3}$ & - & $\mathrm{THF} \mathrm{H}_{2} \mathrm{O}(5: 1, \mathrm{v} / \mathrm{v})$ & 11 & 8 \\
\hline 2 & $\mathrm{PdCl}_{2}$ & $\mathrm{~K}_{2} \mathrm{CO}_{3}$ & - & THF-H ${ }_{2} \mathrm{O}(5: 1, \mathrm{v} / \mathrm{v})$ & 35 & - \\
\hline 3 & $\operatorname{Pd}(\mathrm{TFA})_{2}$ & $\mathrm{~K}_{2} \mathrm{CO}_{3}$ & - & $\mathrm{THF}-\mathrm{H}_{2} \mathrm{O}(5: 1, \mathrm{v} / \mathrm{v})$ & 30 & - \\
\hline 5 & $\mathrm{Pd}(\mathrm{dppf}) \mathrm{Cl}_{2}$ & $\mathrm{~K}_{2} \mathrm{CO}_{3}$ & - & THF-H ${ }_{2} \mathrm{O}(5: 1, \mathrm{v} / \mathrm{v})$ & 32 & 3 \\
\hline 6 & $\operatorname{Pd}(\mathrm{acac})_{2}$ & $\mathrm{~K}_{2} \mathrm{CO}_{3}$ & - & $\mathrm{THF}-\mathrm{H}_{2} \mathrm{O}(5: 1, \mathrm{v} / \mathrm{v})$ & 21 & - \\
\hline 7 & $\mathrm{Pd}_{2} \mathrm{dba}_{3}$ & $\mathrm{~K}_{2} \mathrm{CO}_{3}$ & - & $\mathrm{THF}-\mathrm{H}_{2} \mathrm{O}(5: 1, \mathrm{v} / \mathrm{v})$ & 45 & - \\
\hline 9 & $\begin{array}{l}\text { PEPPSI-IPr } \\
\text { catalyst }\end{array}$ & $\mathrm{K}_{2} \mathrm{CO}_{3}$ & - & $\mathrm{THF}-\mathrm{H}_{2} \mathrm{O}(5: 1, \mathrm{v} / \mathrm{v})$ & 42 & - \\
\hline 10 & $\mathrm{Pd}_{2} \mathrm{dba}_{3}$ & $\mathrm{~K}_{2} \mathrm{CO}_{3}$ & ${ }^{t} \mathrm{Bu}_{3} \mathrm{P}(0.1)$ & THF-H ${ }_{2} \mathrm{O}(5: 1, \mathrm{v} / \mathrm{v})$ & 26 & - \\
\hline 11 & $\operatorname{Pd}(\mathrm{TFA})_{2}$ & $\mathrm{~K}_{2} \mathrm{CO}_{3}$ & ${ }^{t} \mathrm{Bu}_{3} \mathrm{P}(0.1)$ & $\mathrm{THF}-\mathrm{H}_{2} \mathrm{O}(5: 1, \mathrm{v} / \mathrm{v})$ & 7 & - \\
\hline 12 & $\mathrm{Pd}(\mathrm{TFA})_{2}$ & $\mathrm{~K}_{2} \mathrm{CO}_{3}$ & RuPhos (0.1) & $\mathrm{THF}-\mathrm{H}_{2} \mathrm{O}(5: 1, \mathrm{v} / \mathrm{v})$ & 24 & 1 \\
\hline 13 & $\operatorname{Pd}(\mathrm{TFA})_{2}$ & $\mathrm{~K}_{2} \mathrm{CO}_{3}$ & SPhos (0.1) & THF-H ${ }_{2} \mathrm{O}(5: 1, \mathrm{v} / \mathrm{v})$ & 21 & 1 \\
\hline 18 & $\mathrm{Pd}\left(\mathrm{PPh}_{3}\right)_{4}$ & $\mathrm{~K}_{2} \mathrm{CO}_{3}$ & - & THF & 9 & 1 \\
\hline
\end{tabular}




\begin{tabular}{|c|c|c|c|c|c|c|}
\hline 19 & $\mathrm{Pd}\left(\mathrm{PPh}_{3}\right)_{4}$ & $\mathrm{~K}_{2} \mathrm{CO}_{3}$ & - & Dioxane & 13 & 1 \\
\hline $20^{[\mathrm{b}]}$ & $\mathrm{Pd}(\mathrm{OAc})_{2}$ & $\mathrm{~K}_{2} \mathrm{CO}_{3}$ & XPhos (0.3) & Dioxane & 17 & 1 \\
\hline $21^{[\mathrm{b}]}$ & $\mathrm{Pd}(\mathrm{OAc})_{2}$ & $\mathrm{NaO}^{t} \mathrm{Bu}$ & XPhos (0.3) & Dioxane & 67 & - \\
\hline $22^{[\mathrm{b}, \mathrm{c}]}$ & $\mathrm{Pd}(\mathrm{OAc})_{2}$ & $\mathrm{~K}_{2} \mathrm{CO}_{3}$ & XPhos (3) & Dioxane & 37 & - \\
\hline $23^{[\mathrm{b}]}$ & $\mathrm{Pd}(\mathrm{OAc})_{2}$ & $\mathrm{NaO}^{t} \mathrm{Bu}$ & XPhos (0.3) & $\begin{array}{c}{ }^{t} \mathrm{BuOH}-\text { dioxane } \\
(5: 2, \mathrm{v} / \mathrm{v})\end{array}$ & 20 & - \\
\hline $24^{[\mathrm{b}]}$ & $\mathrm{Pd}(\mathrm{OAc})_{2}$ & $\mathrm{NaO}^{t} \mathrm{Bu}$ & XPhos (0.3) & $\begin{array}{c}\text { ACN-dioxane } \\
(5: 2, \mathrm{v} / \mathrm{v})\end{array}$ & 6 & - \\
\hline
\end{tabular}

[a] HPLC yields.

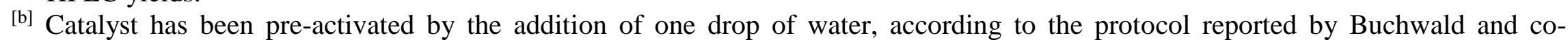
workers. ${ }^{8}$

[c] Stoichiometric reaction with inverse addition. 
General synthetic procedures for tetradentate ligand-containing using the optimized reaction condition $(1,3$ and 4$)$

To a two-necked flask was added with $\mathrm{Pd}(\mathrm{OAc})_{2}$ (0.1 equiv.) and XPhos (0.3 equiv.). The mixture was degassed and then was dissolved in degassed dioxane $(40 \mathrm{~mL} / \mathrm{mmol})$, with the addition of 1 drop of water. The resulting solution was then heated at $90{ }^{\circ} \mathrm{C}$ for several minutes. To another flask were added the chlorogold(III) precursor (1 equiv.), 3,6-di-tert-butyl-1-(4,4,5,5-tetramethyl1,3,2-dioxaborolan-2-yl)-9H-carbazole (1 equiv.) and $\mathrm{NaO}^{t} \mathrm{Bu}$ (3.7 equiv.). The mixture was degassed, and was dissolved in degassed dioxane (100 mL/mmol chlorogold(III) complex). The catalyst mixture was then cannulated into the reaction flask and heated at $90{ }^{\circ} \mathrm{C}$. Then the mixture was concentrated and the product was purified by column chromatography on silica gel with hexane and dichloromethane as eluents. The product was further purified by recrystallization in ethyl acetate.

\section{$\left[\mathrm{Au}\left\{N-{ }^{t} \mathrm{BuCbz}-\mathrm{C}^{\wedge} \mathbf{N}\left({ }^{t} \mathrm{BuC}_{6} \mathrm{H}_{4}\right)^{\wedge} \mathrm{C}^{t} \mathrm{Bu}\right\}\right](\mathbf{1})$}

Orange solid. Yield: $23 \mathrm{mg}, 50 \%$.

\section{$\left[\mathrm{Au}\left\{N-{ }^{t} \mathrm{BuCbz}^{-\mathrm{C}^{\wedge}} \mathbf{N}\left({ }^{t} \mathrm{BuC}_{6} \mathrm{H}_{4}\right)^{\wedge} \mathrm{CF}\right\}\right](3)$}

Deep red solid. Yield: $27 \mathrm{mg}, 62 \% .{ }^{1} \mathrm{H}$ NMR (500 MHz, dichloromethane- $\left.d_{2}, \delta / \mathrm{ppm}\right): \delta 8.20(\mathrm{~d}$, $J=1.9 \mathrm{~Hz}, 1 \mathrm{H},-\mathrm{Cbz}-), 8.12\left(\mathrm{~s}, 1 \mathrm{H},-\mathrm{Cbz}^{-}\right), 8.04\left(\mathrm{~d}, J=8.1 \mathrm{~Hz}, 1 \mathrm{H},-\mathrm{C}_{6} \mathrm{H}_{2}-\right.$ of $\left.\mathrm{C}^{\wedge} \mathrm{N}^{\wedge} \mathrm{C}\right), 8.01$ $(\mathrm{d}, J=2.0 \mathrm{~Hz}, 1 \mathrm{H},-\mathrm{Cbz}-), 7.82\left(\mathrm{dd}, J=9.2 \mathrm{~Hz}, 2.6 \mathrm{~Hz}, 1 \mathrm{H},-\mathrm{C}_{6} \mathrm{H}_{3}-\right.$ of $\left.\mathrm{C}^{\wedge} \mathrm{N}^{\wedge} \mathrm{C}\right), 7.70(\mathrm{~d}, J=8.5$ $\mathrm{Hz}, 1 \mathrm{H},-\mathrm{Cbz}-), 7.52\left(\mathrm{~d}, J=8.0 \mathrm{~Hz}, 2 \mathrm{H},-\mathrm{C}_{6} \mathrm{H}_{4}{ }^{t} \mathrm{Bu}\right.$ of $\left.\mathrm{C}^{\wedge} \mathrm{N}^{\wedge} \mathrm{C}\right), 7.47(\mathrm{dd}, J=8.6 \mathrm{~Hz}, 5.2 \mathrm{~Hz}, 1 \mathrm{H}$, $-\mathrm{C}_{6} \mathrm{H}_{3}-$ of $\left.\mathrm{C}^{\wedge} \mathrm{N}^{\wedge} \mathrm{C}\right), 7.30(\mathrm{dd}, J=8.6 \mathrm{~Hz}, 1.9 \mathrm{~Hz}, 1 \mathrm{H},-\mathrm{Cbz}-), 7.14\left(\mathrm{~d}, J=8.0 \mathrm{~Hz}, 2 \mathrm{H},-\mathrm{C}_{6} \mathrm{H}_{4}{ }^{t} \mathrm{Bu}\right.$ of $\left.\mathrm{C}^{\wedge} \mathrm{N}^{\wedge} \mathrm{C}\right), 6.96\left(\mathrm{~d}, J=8.1 \mathrm{~Hz}, 1 \mathrm{H},-\mathrm{C}_{6} \mathrm{H}_{2}-\right.$ of $\left.\mathrm{C}^{\wedge} \mathrm{N}^{\wedge} \mathrm{C}\right), 6.84\left(\mathrm{td}, J=8.6 \mathrm{~Hz}, 2.6 \mathrm{~Hz}, 1 \mathrm{H},-\mathrm{C}_{6} \mathrm{H}_{3}-\right.$ of $\left.\mathrm{C}^{\wedge} \mathrm{N}^{\wedge} \mathrm{C}\right), 6.78\left(\mathrm{~s}, 1 \mathrm{H},-\mathrm{C}_{5} \mathrm{NH}-\right.$ of $\left.\mathrm{C}^{\wedge} \mathrm{N}^{\wedge} \mathrm{C}\right), 2.63\left(\mathrm{t}, J=7.6 \mathrm{~Hz}, 2 \mathrm{H},-\mathrm{CH}_{2}-\right.$ of $\left.\mathrm{C}^{\wedge} \mathrm{N}^{\wedge} \mathrm{C}\right), 2.36-$ $2.31\left(\mathrm{~m}, 2 \mathrm{H},-\mathrm{CH}_{2}-\right.$ of $\left.\mathrm{C}^{\wedge} \mathrm{N}^{\wedge} \mathrm{C}\right), 1.57\left(\mathrm{~s}, 9 \mathrm{H},-{ }^{t} \mathrm{Bu}\right.$ of $\left.-\mathrm{Cbz}-\right), 1.42\left(\mathrm{~s}, 9 \mathrm{H},-{ }^{t} \mathrm{Bu}\right.$ of $\left.-\mathrm{C}_{6} \mathrm{H}_{4}{ }^{t} \mathrm{Bu}\right)$, $1.41\left(\mathrm{~s}, 9 \mathrm{H},-{ }^{t} \mathrm{Bu}\right.$ of $\left.-\mathrm{Cbz}-\right) ;{ }^{13} \mathrm{C}\left\{{ }^{1} \mathrm{H}\right\}$ NMR (125 MHz, dichloromethane- $\left.d_{2}, \delta / \mathrm{ppm}\right): \delta 173.80$, 173.77, 165.01, 162.99, 159.94, 159.73, 158.94, 153.04, 152.95, 147.21, 145.28, 145.22, 142.25, $140.77,137.90,137.79,134.59,131.21,128.88$, 127.64, 127.34, 126.94, 126.88, 126.44, 126.31, 
126.01, 125.91, 124.83, 124.68, 124.54, 122.30, 118.37, 117.71, 117.38, 116.43, 116.28, 113.65, 113.46, 35.29, 35.26, 35.02, 32.60, 32.44, 31.64, 27.56, 24.68; ${ }^{19} \mathrm{~F}\left\{{ }^{1} \mathrm{H}\right\}$ NMR (471 MHz, dichloromethane- $d_{2}, \delta / \mathrm{ppm}$ ): $\delta-109.51$; HRMS (positive APCI): Found: m/z $879.3299[\mathrm{M}+\mathrm{H}]^{+}$. Calcd for $\mathrm{C}_{49} \mathrm{H}_{47} \mathrm{AuFN}_{2}[\mathrm{M}+\mathrm{H}]^{+}: \mathrm{m} / \mathrm{z} 879.3383$.

\section{$\left[\mathrm{Au}\left\{N-{ }^{t} \mathrm{BuCbz}-\mathrm{C}^{\wedge} \mathrm{N}\left({ }^{t} \mathrm{BuC}_{6} \mathrm{H}_{4}\right)^{\wedge} \mathrm{COMe}\right\}\right](4)$}

Orange solid. Yield: $30 \mathrm{mg}, 66 \% .{ }^{1} \mathrm{H}$ NMR $\left(500 \mathrm{MHz}\right.$, dichloromethane- $\left.d_{2}, \delta / \mathrm{ppm}\right): \delta 8.21$ (d, $J$ $\left.=1.7 \mathrm{~Hz}, 1 \mathrm{H},-\mathrm{Cbz}_{-}\right), 8.17-8.11\left(\mathrm{~m}, 2 \mathrm{H},-\mathrm{C}_{6} \mathrm{H}_{2}-\right.$ of $\left.^{\wedge} \mathrm{C}^{\wedge} \mathrm{C}\right), 8.09\left(\mathrm{~s}, 1 \mathrm{H},-\mathrm{Cbz}^{-}\right), 7.91(\mathrm{~d}, J=$ $\left.8.6 \mathrm{~Hz}, 1 \mathrm{H},-\mathrm{Cbz}_{-}\right), 7.76-7.72\left(\mathrm{~m}, 1 \mathrm{H},-\mathrm{C}_{6} \mathrm{H}_{3}-\right.$ of $\left.\mathrm{C}^{\wedge} \mathrm{N}^{\wedge} \mathrm{C}\right), 7.48\left(\mathrm{~d}, J=7.7 \mathrm{~Hz}, 2 \mathrm{H},-\mathrm{C}_{6} \mathrm{H}_{4}{ }^{t} \mathrm{Bu}\right.$ of $\left.\mathrm{C}^{\wedge} \mathrm{N}^{\wedge} \mathrm{C}\right), 7.41\left(\mathrm{~d}, J=8.4 \mathrm{~Hz}, 1 \mathrm{H},-\mathrm{C}_{6} \mathrm{H}_{3}-\right.$ of $\left.\mathrm{C}^{\wedge} \mathrm{N}^{\wedge} \mathrm{C}\right), 7.33\left(\mathrm{~d}, J=8.6 \mathrm{~Hz}, 1 \mathrm{H},-\mathrm{Cbz}^{-}\right), 7.15(\mathrm{~d}, J$ $=7.7 \mathrm{~Hz}, 2 \mathrm{H},-\mathrm{C}_{6} \mathrm{H}_{4}{ }^{t} \mathrm{Bu}$ of $\left.\mathrm{C}^{\wedge} \mathrm{N}^{\wedge} \mathrm{C}\right), 7.05\left(\mathrm{~d}, J=7.8 \mathrm{~Hz}, 1 \mathrm{H},-\mathrm{C}_{6} \mathrm{H}_{2}-\right.$ of $\left.\mathrm{C}^{\wedge} \mathrm{N}^{\wedge} \mathrm{C}\right), 6.86(\mathrm{~s}, 1 \mathrm{H}$, $-\mathrm{C}_{5} \mathrm{NH}-$ of $\left.\mathrm{C}^{\wedge} \mathrm{N}^{\wedge} \mathrm{C}\right), 6.70\left(\mathrm{~d}, J=8.4 \mathrm{~Hz}, 1 \mathrm{H},-\mathrm{C}_{6} \mathrm{H}_{3}-\right.$ of $\left.\mathrm{C}^{\wedge} \mathrm{N}^{\wedge} \mathrm{C}\right), 3.62\left(\mathrm{~s}, 3 \mathrm{H},-\mathrm{OCH}_{3}\right), 2.73-2.69$ (m, $2 \mathrm{H},-\mathrm{CH}_{2}-$ of $\left.\mathrm{C}^{\wedge} \mathrm{N}^{\wedge} \mathrm{C}\right), 2.53-2.46\left(\mathrm{~m}, 2 \mathrm{H},-\mathrm{CH}_{2}-\right.$ of $\left.\mathrm{C}^{\wedge} \mathrm{N}^{\wedge} \mathrm{C}\right), 1.58\left(\mathrm{~s}, 9 \mathrm{H},-{ }^{t} \mathrm{Bu}\right.$ of $\left.-\mathrm{Cbz}-\right)$, $1.44\left(\mathrm{~s}, 9 \mathrm{H},-{ }^{t} \mathrm{Bu}\right.$ of $\left.-\mathrm{Cbz}-\right), 1.40\left(\mathrm{~s}, 9 \mathrm{H},-{ }^{t} \mathrm{Bu}\right.$ of $\left.-\mathrm{C}_{6} \mathrm{H}_{4}{ }^{t} \mathrm{Bu}\right) ;{ }^{13} \mathrm{C}\left\{{ }^{1} \mathrm{H}\right\}$ NMR (125 MHz, dichloromethane- $\left.d_{2}, \delta / \mathrm{ppm}\right): \delta 172.76,161.24,160.83,160.36,160.09,153.35,152.80,145.54$, $145.40,143.32$, 142.25, 140.81, 138.23, 137.65, 135.80, 134.91, 131.55, 128.85, 127.34, 126.86, 126.76, 126.62, 126.35, 125.95, 124.77, 122.32, 121.96, 118.58, 118.19, 117.13, 116.35, 116.01, 113.64, 35.27, 35.26, 35.04, 32.61, 32.42, 31.64, 27.62, 24.92; HRMS (positive APCI): Found : $\mathrm{m} / \mathrm{z} 891.3504[\mathrm{M}+\mathrm{H}]^{+}$. Calcd for $\mathrm{C}_{50} \mathrm{H}_{50} \mathrm{AuN}_{2} \mathrm{O}[\mathrm{M}+\mathrm{H}]^{+}: \mathrm{m} / \mathrm{z} 891.3589$. 


\section{Synthesis of Control Tridentate Cyclometalated Gold(III) Complex}

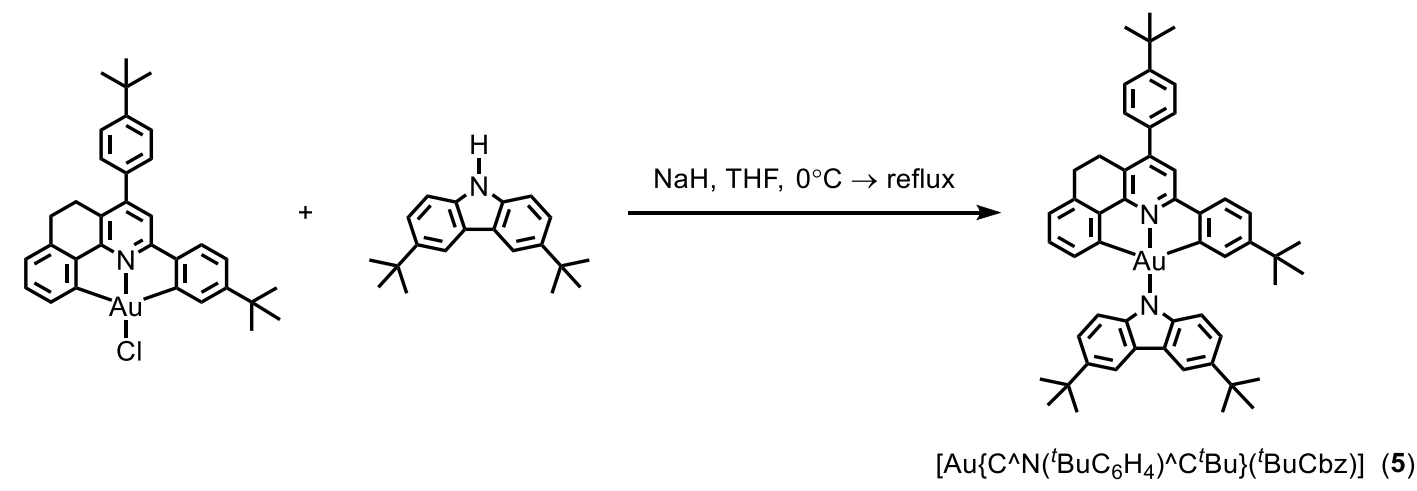

Scheme S2 Synthetic route of the tridentate cyclometalated gold(III) complex 5.

\section{$\left[\mathrm{Au}\left\{\mathrm{C}^{\wedge} \mathrm{N}\left({ }^{t} \mathrm{BuC}_{6} \mathrm{H}_{4}\right){ }^{\wedge} \mathrm{C}^{t} \mathrm{Bu}\right\}\left({ }^{t} \mathrm{BuCbz}\right)\right](5)$}

3,6-Di-tert-butyl-9H-carbazole (68 $\mathrm{mg}, 0.24 \mathrm{mmol}$ ) was added to a flask and degassed. Degassed anhydrous tetrahydrofuran $(5 \mathrm{~mL})$ was added. $\mathrm{NaH}(11 \mathrm{mg}, 0.26 \mathrm{mmol}, 60 \mathrm{wt} \%$ in mineral oil $)$ was added slowly. The mixture was stirred at room temperature for 30 minutes. To another flask was added $\left[\mathrm{Au}\left\{\mathrm{C}^{\wedge} \mathrm{N}\left({ }^{t} \mathrm{BuC}_{6} \mathrm{H}_{4}\right)^{\wedge} \mathrm{C}^{t} \mathrm{Bu}\right\} \mathrm{Cl}\right](150 \mathrm{mg}, 0.22 \mathrm{mmol})$. The solid was degassed and dissolved in degassed anhydrous tetrahydrofuran $(40 \mathrm{~mL})$. The resulting solution was then cannulated into the flask containing deprotonated carbazole. The mixture was stirred at room temperature for 30 minutes and then heated to reflux overnight. Then it was quenched with water and concentrated. The product was purified by column chromatography on silica gel with a mixture of hexane and dichloromethane as eluent. The product was further purified by re-crystallization from a mixture of tetrahydrofuran and ethyl acetate.

Orange solid. Yield: $55 \mathrm{mg}, 27 \% .{ }^{1} \mathrm{H}$ NMR (500 MHz, dichloromethane-d2, $\left.\delta / \mathrm{ppm}\right): \delta 8.17$ (s, 2H, $-\mathrm{Cbz}-), 7.60$ (d, J = 7.8 Hz, 2H, $\left.-\mathrm{C}_{6} \mathrm{H}_{4}-\right), 7.50$ (d, J = 8.1 Hz, 1H, $\left.-\mathrm{C}_{6} \mathrm{H}_{3}-\right), 7.45$ (d, J = $7.8 \mathrm{~Hz}$, $\left.2 \mathrm{H},-\mathrm{C}_{6} \mathrm{H}_{4}-\right)$, 7.42-7.38 (m, 3H, $-\mathrm{Cbz}-$ and $\left.-\mathrm{C}_{5} \mathrm{HN}-\right), 7.35$ (d, J = 8.7 Hz, 2H, -Cbz-), 7.17 (d, $\left.J=8.1 \mathrm{~Hz}, 1 \mathrm{H},-\mathrm{C}_{6} \mathrm{H}_{3}-\right), 7.11\left(\mathrm{t}, J=7.3 \mathrm{~Hz}, 1 \mathrm{H},-\mathrm{C}_{6} \mathrm{H}_{3}-\right), 7.00\left(\mathrm{~d}, J=7.3 \mathrm{~Hz}, 1 \mathrm{H},-\mathrm{C}_{6} \mathrm{H}_{3}-\right), 6.94$ $\left(\mathrm{d}, J=7.3 \mathrm{~Hz}, 1 \mathrm{H},-\mathrm{C}_{6} \mathrm{H}_{3}-\right), 6.72\left(\mathrm{~s}, 1 \mathrm{H},-\mathrm{C}_{6} \mathrm{H}_{3}-\right), 3.08-3.00\left(\mathrm{~m}, 4 \mathrm{H},-\mathrm{CH}_{2}-\right), 1.46\left(\mathrm{~s}, 18 \mathrm{H},-{ }^{t} \mathrm{Bu}\right.$ of $\mathrm{Cbz}), 1.41\left(\mathrm{~s}, 9 \mathrm{H},-{ }^{t} \mathrm{Bu}\right.$ of $\left.\mathrm{C}^{\wedge} \mathrm{N}^{\wedge} \mathrm{C}\right), 0.90\left(\mathrm{~s}, 9 \mathrm{H},-{ }^{t} \mathrm{Bu}\right.$ of $\left.\mathrm{C}^{\wedge} \mathrm{N}^{\wedge} \mathrm{C}\right) ;{ }^{13} \mathrm{C}\left\{{ }^{1} \mathrm{H}\right\}$ data were not available due to the low solubility of the compound; HRMS (positive ESI): Found : m/z 919.4221 $[\mathrm{M}+\mathrm{H}]^{+}$. Calcd for $\mathrm{C}_{53} \mathrm{H}_{58} \mathrm{AuN}_{2}[\mathrm{M}+\mathrm{H}]^{+}: \mathrm{m} / \mathrm{z} 919.4260$. 


\section{Thermogravimetric Traces}

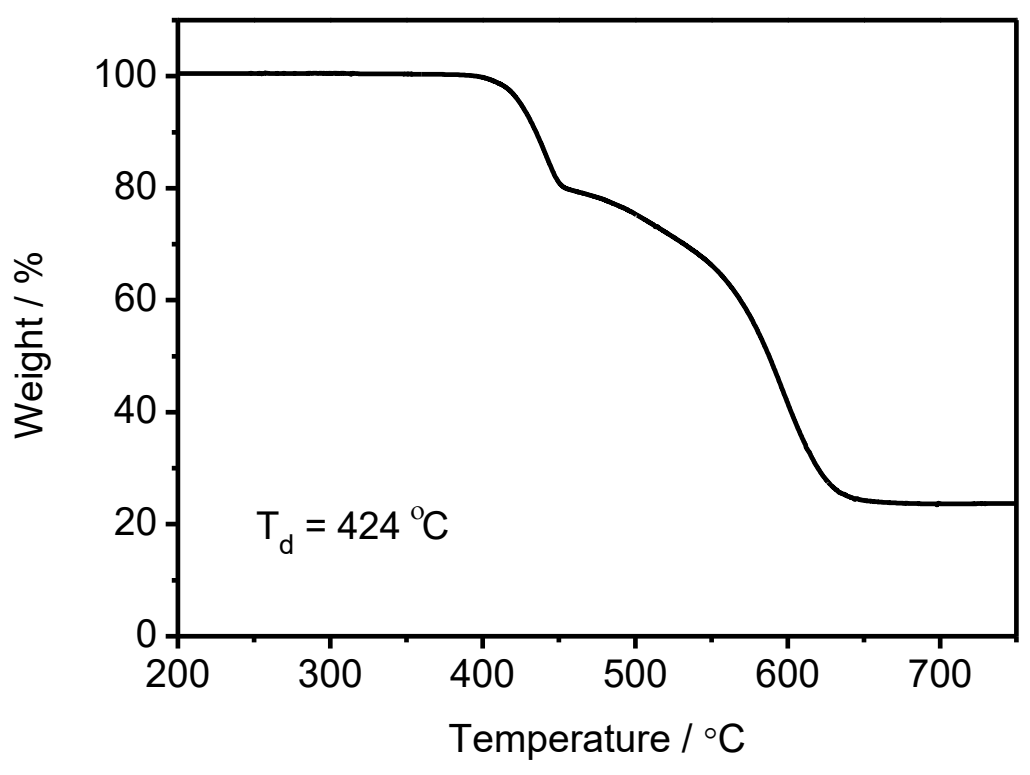

Figure S3 TGA trace of $\mathbf{1}$.

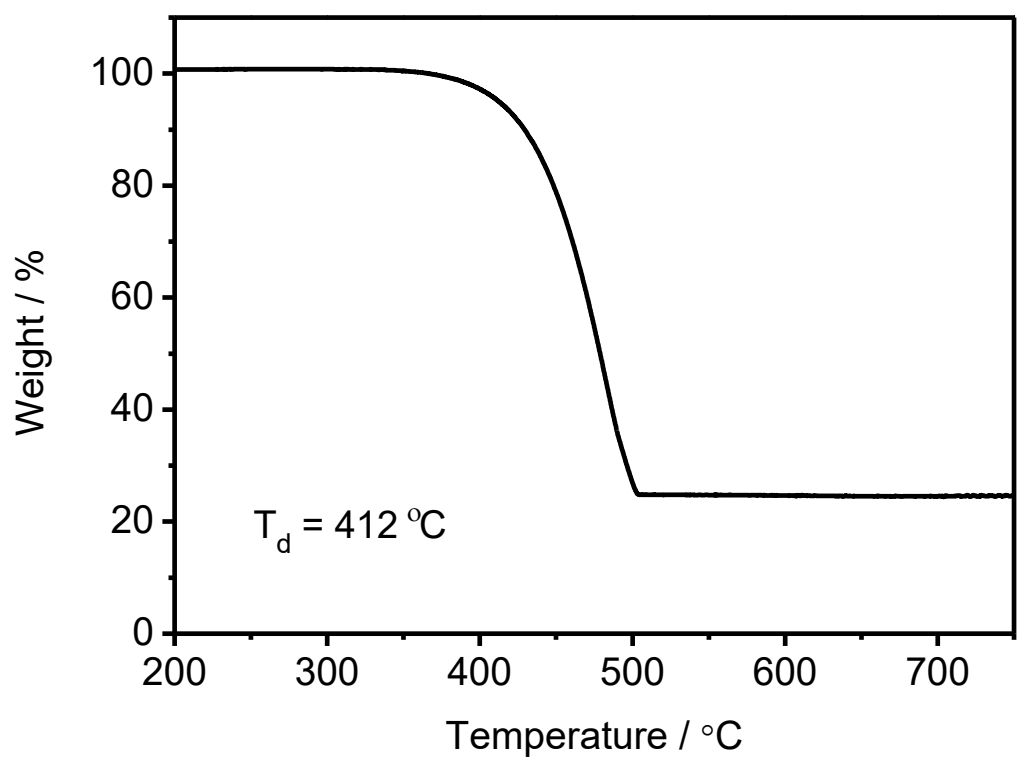

Figure S4 TGA trace of 2 . 


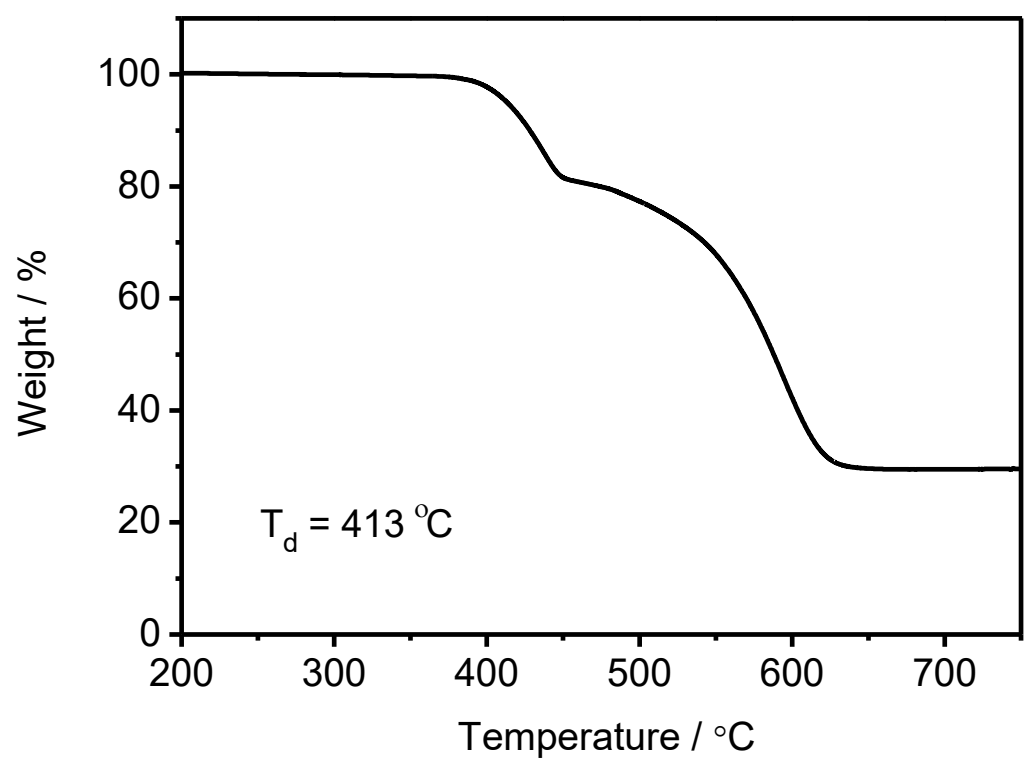

Figure S5 TGA trace of 3.

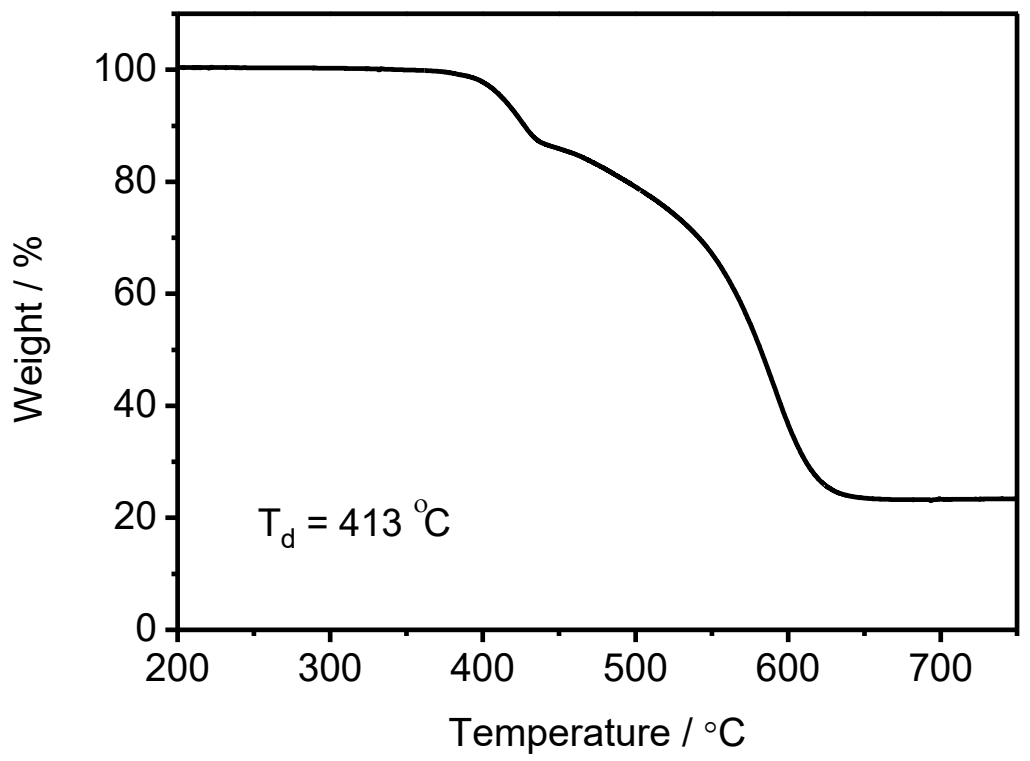

Figure S6 TGA trace of 4 . 


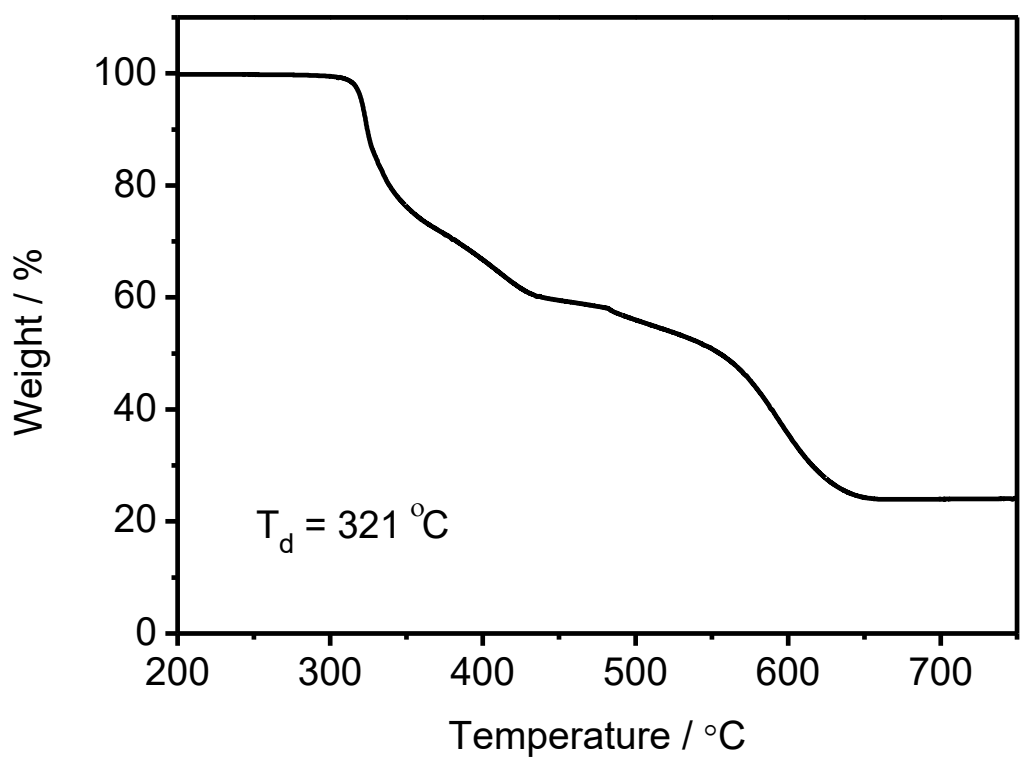

Figure S7 TGA trace of $\mathbf{5}$. 


\section{X-Ray Crystal Structure Data and Refinement Details}

Table S3 Crystal data and structure refinement details for $\mathbf{1}$.

Empirical formula

Formula weight

Temperature / $\mathrm{K}$

Crystal system

Space group

$a / \AA$

$b / \AA$

$c / \AA$

$\alpha /^{\circ}$

$\beta /{ }^{\circ}$

$\gamma /{ }^{\circ}$

Volume / $\AA^{3}$

$Z$

Density (calcd) / $\mathrm{g} \mathrm{cm}^{-3}$

Crystal size / $\mathrm{mm}$

$\mu$ (synchrotron) $/ \mathrm{mm}^{-1}$

$F(000)$

Color / habit

Radiation

$2 \theta$ range for data collection $/^{\circ}$

Index ranges

Reflections collected

Independent reflections

Data/restraints/parameters

$R_{1}{ }^{[\mathrm{a}]}, w R_{2}^{[\mathrm{b}]}(I>2 \sigma(I))$

Goodness-of-fit on $F^{2[\mathrm{c}]}$

Largest diff. peak/hole / e $\AA^{-3}$

${ }^{[\mathrm{a}]} R_{1}=\Sigma|| F_{\text {obs }}|-| F_{\text {calc }}|| /|| F_{\text {obs }} \mid$

${ }^{[b]} w R_{2}=\left[\Sigma w\left(\left|F_{o b s}{ }^{2}\right|-\left|F_{c a l c}{ }^{2}\right|\right)^{2} / \Sigma w\left|F_{o b s}{ }^{2}\right|^{2}\right]^{1 / 2}$

${ }^{[\mathrm{c}]}$ Goodness-of-fit $=\left[\Sigma w\left(\left|F_{\text {obs }}{ }^{2}\right|-\left|F_{\text {calc }}{ }^{2}\right|\right)^{2} /\left(\mathrm{N}_{\text {obs }}-\mathrm{N}_{\text {param }}\right)\right]^{1 / 2}$
$\mathrm{C}_{53} \mathrm{H}_{55} \mathrm{AuN}_{2}$

916.96

100(2)

Orthorhombic

$\mathrm{Pbca}$

21.975(4)

$22.822(5)$

$50.375(10)$

90

90

90

25264(9)

24

1.446

$0.20 \times 0.18 \times 0.16$

5.139

11184

Orange / block

Synchrotron $(\lambda=0.82653 \AA)$

3.136 to 59.818

$-26 \leq h \leq 0$

$-27 \leq k \leq 27$

$-60 \leq l \leq 60$

82047

$23156\left(\mathrm{R}_{\text {int }}=0.0900, \mathrm{R}_{\text {sigma }}=0.0603\right)$

23156/85/1546

$0.0440,0.1133$

1.044

$1.32 /-2.33$ 
Table S4 Selected bond lengths and angles of $\mathbf{1}$ with estimated standard deviations (esds) in parentheses.

\begin{tabular}{cc}
\hline \multicolumn{3}{c}{ Bond Lengths / $\AA$} \\
Au1-N1 & $1.987(5)$ \\
Au1-N2 & $2.014(5)$ \\
Au1-C19 & $2.106(5)$ \\
Au1-C33 & $2.057(5)$ \\
\hline \multicolumn{3}{c}{ Bond angles / ${ }^{\circ}$} \\
\hline N1-Au1-N2 & $170.37(17)$ \\
N1-Au1-C33 & $79.25(18)$ \\
N2-Au1-C19 & $81.9(2)$ \\
N2-Au1-C33 & $79.25(18)$ \\
\hline
\end{tabular}


Table S5 Crystal data and structure refinement details for 2.

Empirical formula

Formula weight

Temperature / K

Crystal system

Space group

$a / \AA$

$b / \AA$

$c / \AA$

$\alpha /^{\circ}$

$\beta /^{\circ}$

$\gamma /{ }^{\circ}$

Volume / $\AA^{3}$

Z

Density (calcd) / $\mathrm{g} \mathrm{cm}^{-3}$

Crystal size / $\mathrm{mm}$

$\mu$ (synchrotron) $/ \mathrm{mm}^{-1}$

$F(000)$

Color / habit

Radiation

$2 \theta$ range for data collection $/^{\circ}$

Index ranges

Reflections collected

Independent reflections

Data/restraints/parameters

$R_{1}{ }^{[\mathrm{a}]}, w R_{2}{ }^{[\mathrm{b}]}(I>2 \sigma(I))$

Goodness-of-fit on $F^{2[\mathrm{c}]}$

Largest diff. peak/hole / e $\AA^{-3}$

${ }^{\text {a] }} R_{1}=\Sigma|| F_{\text {obs }}|-| F_{\text {calc }}|| / \Sigma\left|F_{\text {obs }}\right|$

${ }^{[b]} w R_{2}=\left[\Sigma w\left(\left|F_{o b s}{ }^{2}\right|-\left|F_{c a l c}{ }^{2}\right|\right)^{2} / \Sigma w\left|F_{o b s}\right|^{2}\right]^{1 / 2}$

${ }^{[\mathrm{c}]}$ Goodness-of-fit $=\left[\Sigma w\left(\left|F_{\text {obs }}{ }^{2}\right|-\left|F_{\text {calc }}{ }^{2}\right|\right)^{2} /\left(\mathrm{N}_{\mathrm{obs}}-\mathrm{N}_{\text {param }}\right)\right]^{1 / 2}$
$\mathrm{C}_{53} \mathrm{H}_{55} \mathrm{AuN}_{2}$

916.96

100(2)

Monoclinic

$\mathrm{P} 2{ }_{1} / n$

15.304(3)

$13.575(3)$

20.004(4)

90

95.89(3)

90

4133.9(15)

4

1.473

$0.18 \times 0.17 \times 0.14$

5.234

1864.0

Orange / block

Synchrotron $(\lambda=0.82653 \mathrm{~nm})$

5.39 to 59.714

$-18 \leq h \leq 17$

$-16 \leq k \leq 0$

$-24 \leq l \leq 24$

13155

$7345\left(R_{\text {int }}=0.0464, R_{\text {sigma }}=0.0427\right)$

$7345 / 4 / 517$

$0.0495,0.1403$

1.079

$1.65 /-3.34$ 
Table S6 Selected bond lengths and angles of $\mathbf{2}$ with estimated standard deviations (esds) in parentheses.

\begin{tabular}{cc}
\hline \multicolumn{3}{c}{ Bond Lengths / $\AA$} \\
Au1-N1 & $2.026(4)$ \\
Au1-C19 & $2.140(4)$ \\
Au1-C33 & $2.052(4)$ \\
Au1-C49 & $2.010(4)$ \\
\hline \multicolumn{3}{c}{ B1-Au1-C49 } & $170.80(15)$ \\
N1-Au1-C19 & $78.73(16)$ \\
N1-Au1-C33 & $81.65(16)$ \\
C33-Au1-C49 & $90.91(16)$ \\
C19-Au1-C49 & $108.76(16)$ \\
\hline
\end{tabular}




\section{Photophysical Studies of 1 and 2}

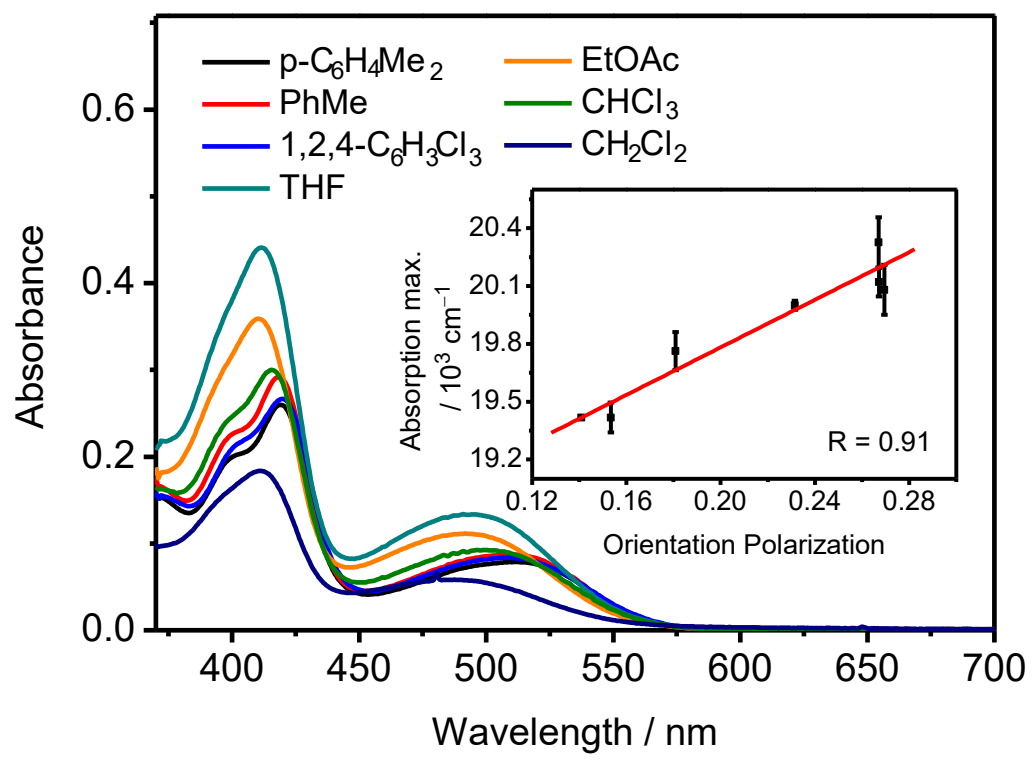

Figure S8 Solvent-dependent UV-visible absorption spectra of $\mathbf{1}$ and plot of lowest-energy absorption maxima versus orientation polarization of solvents in the inset.

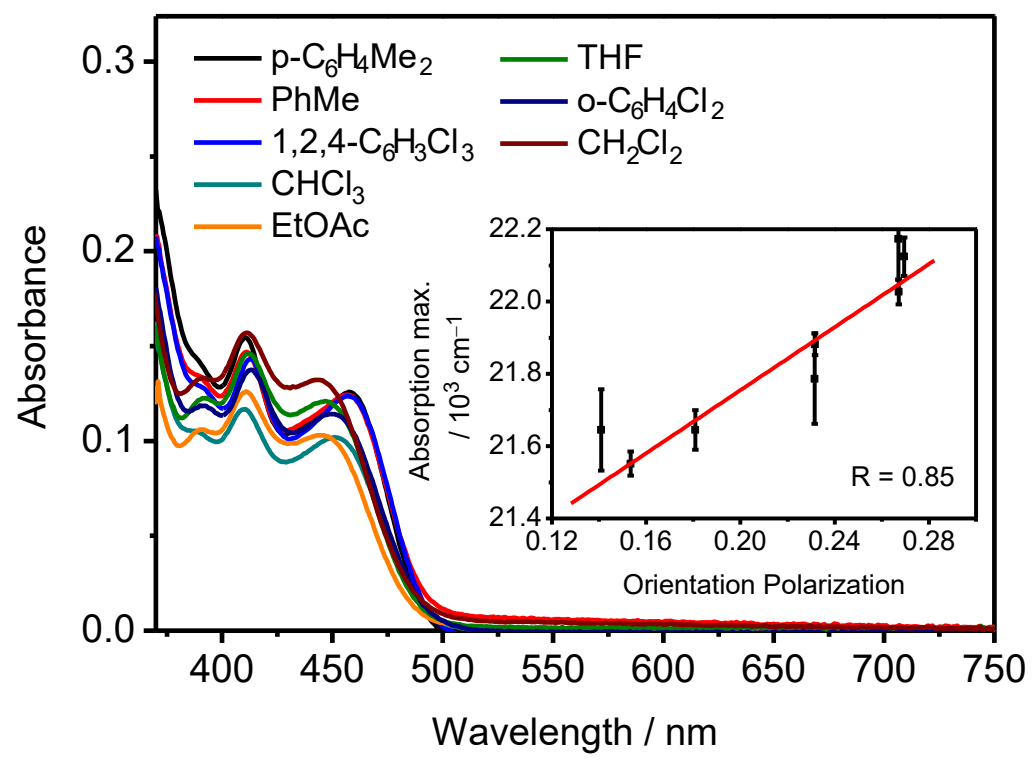

Figure S9 Solvent-dependent UV-visible absorption spectra of $\mathbf{2}$ and plot of lowest-energy absorption maxima versus orientation polarization of solvents in the inset. 


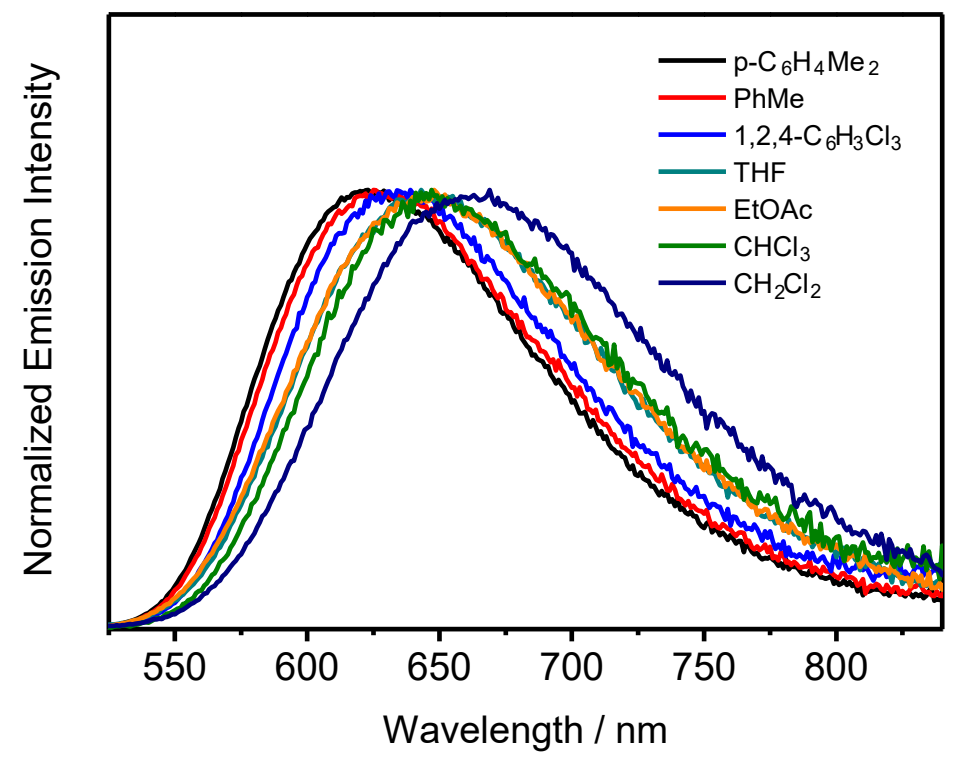

Figure S10 Normalized solvent-dependent emission spectra of 1 at $298 \mathrm{~K}$.

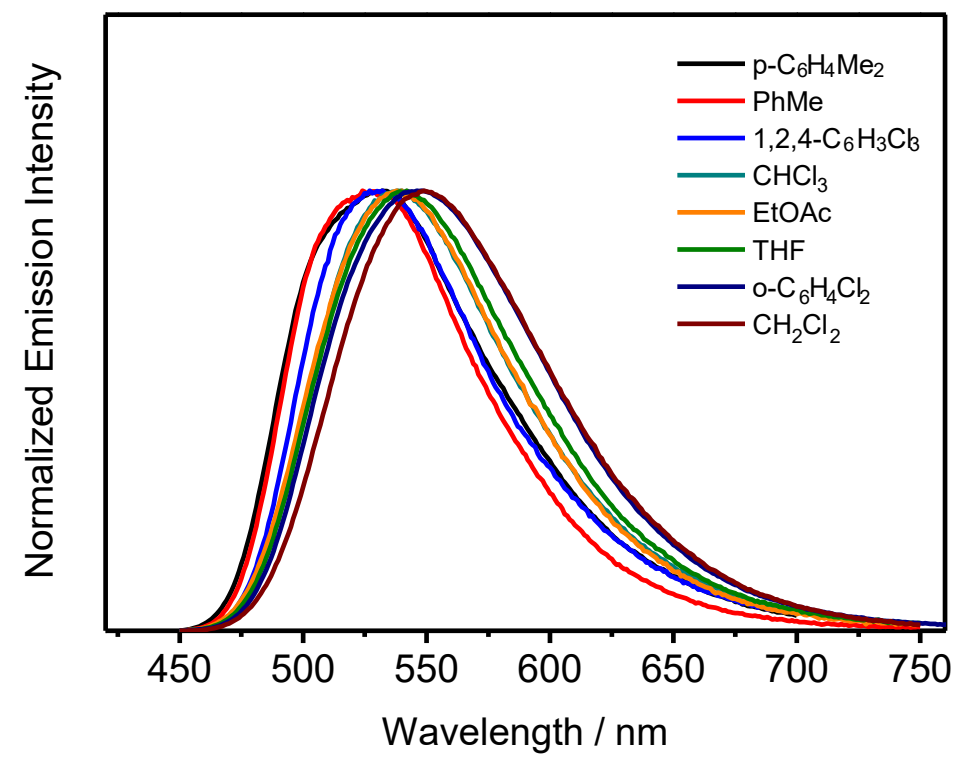

Figure S11 Normalized solvent-dependent emission spectra of 2 at 298 K. 


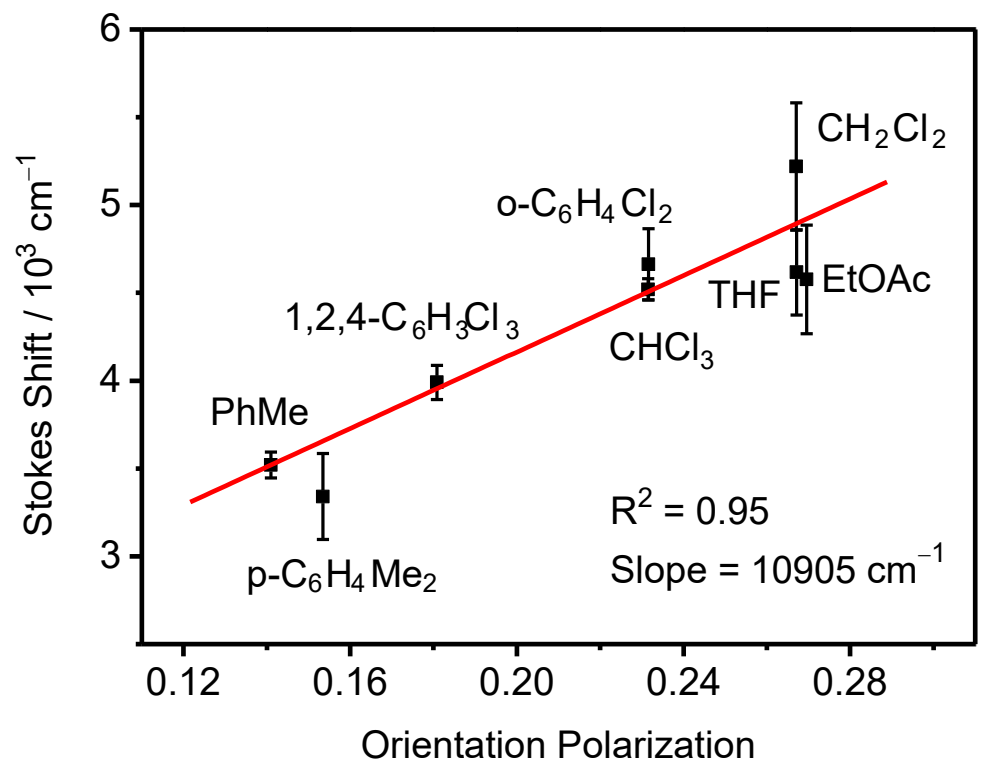

Figure S12 Lippert-Mataga plot of 1 at 298 K.

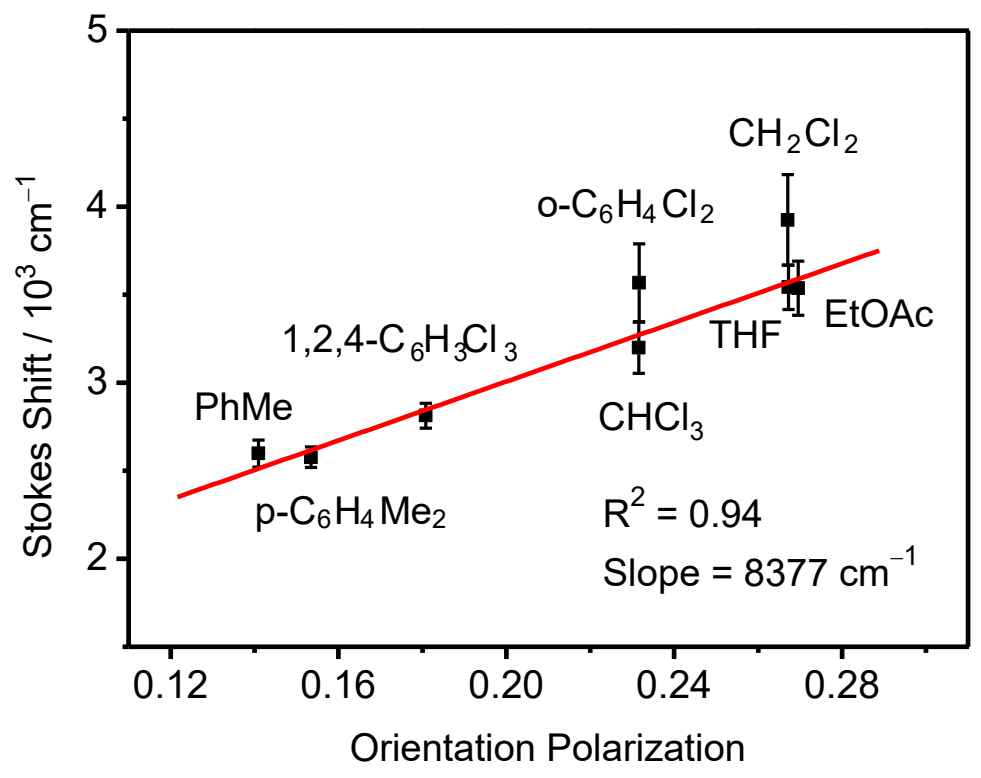

Figure S13 Lippert-Mataga plot of 2 at 298 K. 


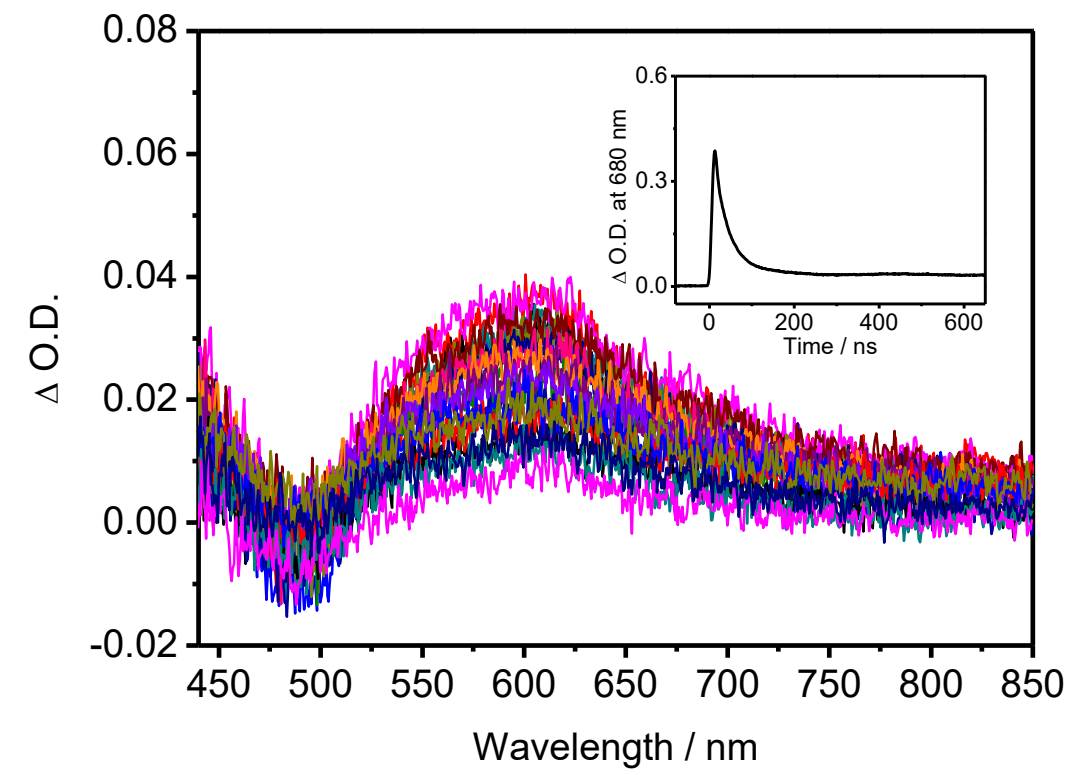

Figure S14 Transient absorption spectra of $\mathbf{1}$ in degassed dichloromethane at 298K at decay times of $0-500 \mathrm{~ns}$ and the decay trace monitored at $680 \mathrm{~nm}$ in the inset.

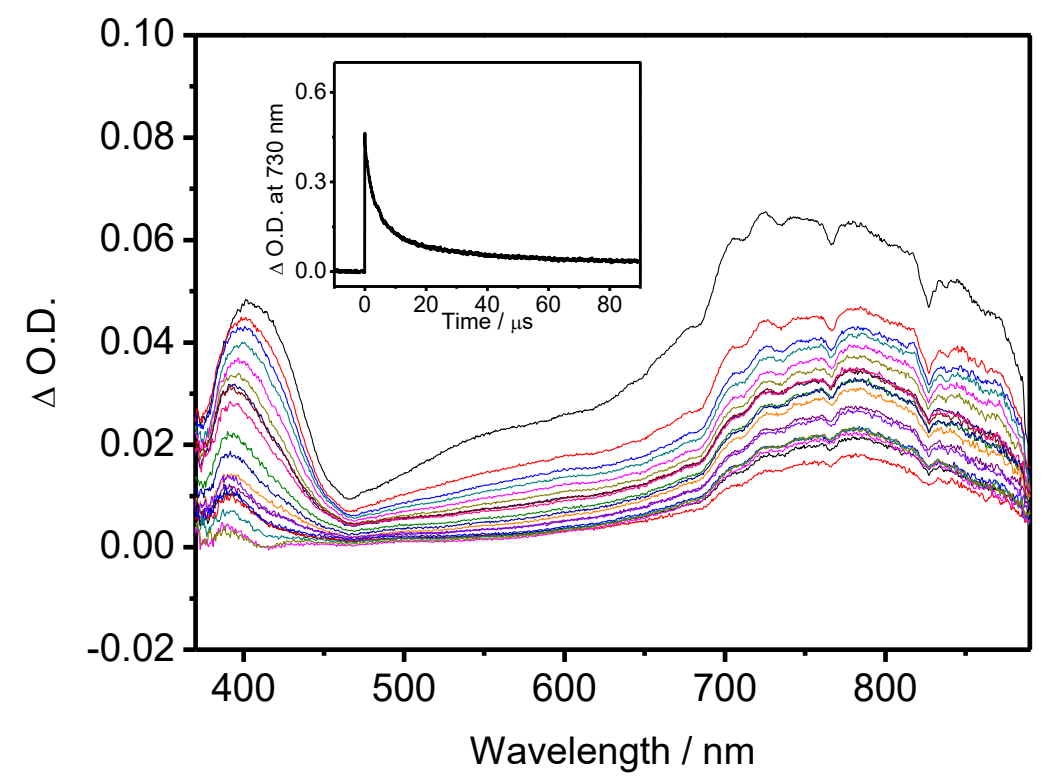

Figure S15 Transient absorption spectra of $\mathbf{2}$ in degassed dichloromethane at $298 \mathrm{~K}$ at decay times of $0-10 \mu$ s and the decay trace monitored at $730 \mathrm{~nm}$ in the inset. 


\section{Photophysical Data}

Table S7 Photophysical properties of complexes 1-5.

\begin{tabular}{|c|c|c|c|c|c|}
\hline Complex & $\begin{array}{l}\text { Absorption } \\
\lambda_{\max } / \mathrm{nm}\left(\varepsilon_{\max } / \mathrm{dm}^{3} \mathrm{~mol}^{-1} \mathrm{~cm}^{-1}\right)\end{array}$ & Medium (Temperature / K) & $\begin{array}{l}\text { Emission } \\
\lambda_{\max } / \mathrm{nm}\left(\tau_{0} / \mu \mathrm{s}\right)\end{array}$ & $\Phi_{\mathrm{PL}}(\operatorname{soln})^{[\mathrm{a}]}$ & $\Phi_{\mathrm{PL}}(\text { film })^{[\mathrm{b}]}$ \\
\hline \multirow[t]{16}{*}{1} & $\begin{array}{l}309 \text { sh (23360), } 392 \text { sh (7930), } \\
410 \text { (9920), } 496 \text { (2990) }\end{array}$ & $\mathrm{CH}_{2} \mathrm{Cl}_{2}(298)$ & $662(<0.1)$ & $2.0 \times 10^{-3}$ & \\
\hline & & $\mathrm{CHCl}_{3}(298)$ & $646(<0.1)$ & $1.8 \times 10^{-3}$ & \\
\hline & & Ethyl acetate (298) & $645(<0.1)$ & $2.0 \times 10^{-3}$ & \\
\hline & & THF (298) & $645(<0.1)$ & $3.9 \times 10^{-3}$ & \\
\hline & & Toluene (298) & $629(0.11)$ & $6.8 \times 10^{-3}$ & \\
\hline & & $p$-Xylene (298) & $622(<0.1)$ & $9.3 \times 10^{-3}$ & \\
\hline & & $o-\mathrm{C}_{6} \mathrm{H}_{4} \mathrm{Cl}_{2}(298)$ & $652(<0.1)$ & $6.0 \times 10^{-3}$ & \\
\hline & & $1,2,4-\mathrm{C}_{6} \mathrm{H}_{3} \mathrm{Cl}_{3}(298)$ & $634(<0.1)$ & $5.8 \times 10^{-3}$ & \\
\hline & & Glass $(77)^{[\mathrm{cc}]}$ & $573(124)$ & & \\
\hline & & Solid (298) & $614(0.2)$ & & \\
\hline & & Solid $(77)^{[\mathrm{d}]}$ & $550,598,654 \operatorname{sh}(27)$ & & \\
\hline & & Thin Film (298) & & & \\
\hline & & $5 \%$ in $\mathrm{MCP}$ & $578(3.7)$ & & 0.21 \\
\hline & & $10 \%$ in $\mathrm{MCP}$ & $582(3.0)$ & & 0.24 \\
\hline & & $20 \%$ in $\mathrm{MCP}$ & $590(2.7)$ & & 0.21 \\
\hline & & $50 \%$ in $\mathrm{MCP}$ & $593(2.1)$ & & 0.16 \\
\hline \multirow[t]{2}{*}{2} & $\begin{array}{l}297 \text { (48095), } 321 \operatorname{sh}(11700), \\
360(10120), 410(5960), \\
450 \operatorname{sh}(4570)\end{array}$ & $\mathrm{CH}_{2} \mathrm{Cl}_{2}(298)$ & $548(3.3)$ & $2.0 \times 10^{-2}$ & \\
\hline & & $\mathrm{CHCl}_{3}(298)$ & $538(4.2)$ & $7.9 \times 10^{-2}$ & \\
\hline
\end{tabular}




Ethyl acetate (298)
THF (298)
Toluene (298)
$p$-Xylene (298)
$o-\mathrm{C}_{6} \mathrm{H}_{4} \mathrm{Cl}_{2}(298)$
$1,2,4-\mathrm{C}_{6} \mathrm{H}_{3} \mathrm{Cl}_{3}{ }^{[\mathrm{d}]}(298)$
Glass $(77)^{[\mathrm{c}, \mathrm{d}]}$
Solid $(298)$
Solid (77)
Thin Film $(298)$
$5 \%$ in MCP
$10 \%$ in MCP
$20 \%$ in MCP
$50 \%$ in MCP

$3 \quad 307 \mathrm{sh}(29300), 389 \mathrm{sh}(9710)$, 410 (12480), 494 (4385)

\section{$\mathrm{CH}_{2} \mathrm{Cl}_{2}$ (298)}

Glass (77) $)^{[\mathrm{c}, \mathrm{d}]}$

Solid (298)

Solid (77) ${ }^{[\mathrm{d}]}$

Thin Film (298)

$5 \%$ in MCP

$10 \%$ in $\mathrm{MCP}$

$20 \%$ in $\mathrm{MCP}$

$50 \%$ in $\mathrm{MCP}$
538 (2.2)

541 (45)

525 (2.9)

527 (3.2)

546 (5.2)

$531(5.3,0.66)$

517,553 sh (197)

$546(0.5)$

$558,593 \mathrm{sh}(65)$

$519(50)$

520 (37)

522 (32)

522 (26)

$667(<0.1)$

553,589 sh (124)

619 (0.2)

$593,645,706$ sh (20)

576 (1.9)

$583(2.0)$

587 (1.5)

$590(1.2)$
$4.1 \times 10^{-2}$

0.11

$6.0 \times 10^{-2}$

$2.6 \times 10^{-2}$

0.12

$1.0 \times 10^{-2}$

0.60

0.52

0.43

0.40

$4.0 \times 10^{-4}$

0.11

0.10

0.07

0.08 
311 sh (393850), 334 (16435),

388 sh (10900), 408 (16845),

497 (4980)

Glass (77)
Solid (298)
Solid (77)

Solid (77)

Thin Film (298)

$5 \%$ in $\mathrm{MCP}$

$10 \%$ in $\mathrm{MCP}$

$20 \%$ in MCP

$50 \%$ in $\mathrm{MCP}$

$\mathrm{CH}_{2} \mathrm{Cl}_{2}$ (298)

Glass (77) ${ }^{[\mathrm{cc}}$

Solid (298)

Solid (77) ${ }^{[\mathrm{d}]}$

Thin Film (298)

$5 \%$ in $\mathrm{MCP}$

$10 \%$ in $\mathrm{MCP}$

$20 \%$ in $\mathrm{MCP}$

$50 \%$ in MCP
$653(<0.1)$

$9.0 \times 10^{-4}$

551,577 sh (130)

$620(0.2)$

$625(55)$

$571(2.8)$

0.17

$575(2.5)$

0.15

$583(2.1)$

0.11

$585(1.7)$

0.09

733

$<10^{-4}$
583(15)

Non-emissive

$585,628(5.8)$

600

0.05

$603-0.04$

$612 \quad 0.03$

$615 \quad 0.03$

\footnotetext{
${ }^{[a]}$ Relative solution luminescence quantum yield, measured at room temperature using degassed acetonitrile solution of $\left[\mathrm{Ru}(\mathrm{bpy})_{3}\right] \mathrm{Cl}_{2}$ as reference (excitation wavelength $=436 \mathrm{~nm}, \Phi_{\mathrm{PL}}=0.06$ ).

${ }^{[b]}$ Absolute luminescence quantum yield of thin films was measured with $350 \mathrm{~nm}$ excitation.

${ }^{[c]}$ Measured in butyronitrile glass.

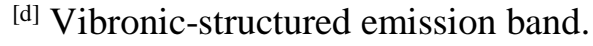




\section{Electrochemical Properties}

Table S8 Electrochemical data for complexes 1-5. ${ }^{[a]}$

\begin{tabular}{ccccc}
\hline Complex & $\begin{array}{c}\text { Oxidation } \\
\begin{array}{c}E_{1 / 2} / \mathrm{V} v s . \mathrm{SCE}^{[\mathrm{b}]} \\
\left(\Delta E_{\mathrm{p}} / \mathrm{mV}\right)^{[\mathrm{c}]}\end{array}\end{array}$ & $\begin{array}{c}\text { Reduction } \\
E_{1 / 2} / \mathrm{V} v s . \mathrm{SCE} \\
\left(\Delta E_{\mathrm{p}} / \mathrm{mV}\right)^{[\mathrm{c}]}\end{array}$ & $\begin{array}{c}E_{\mathrm{HOMO}} / \\
\mathrm{eV}^{[\mathrm{f}]}\end{array}$ & $\begin{array}{c}E_{\mathrm{LUMO}} / \\
\mathrm{eV}^{[\mathrm{f}]}\end{array}$ \\
\hline $\mathbf{1}$ & $+0.80(78)$ & $-1.54(60),[-1.89]$ & -5.60 & -3.26 \\
$\mathbf{2}$ & {$[+1.13]$} & $-1.73]$ & -5.93 & -3.07 \\
$\mathbf{3}$ & $+0.83(80)$ & $-1.51(85),[-1.83]$ & -5.63 & -3.29 \\
$\mathbf{4}$ & $+0.81(87)$ & $-1.54(65),[-1.82]$ & -5.61 & -3.26 \\
$\mathbf{5}$ & $+0.69(81)$ & $-1.53(63),[-1.91]$ & -5.50 & -3.27 \\
\hline
\end{tabular}

${ }^{[a]}$ In dichloromethane solution with $0.1 \mathrm{M}^{n} \mathrm{Bu}_{4} \mathrm{NPF}_{6}$ as supporting electrolyte at $298 \mathrm{~K}$. Working electrode: glassy carbon, scan rate $=100 \mathrm{mVs}^{-1}$.

${ }^{[b]} E_{1 / 2}=\left(E_{\mathrm{pa}}+E_{\mathrm{pc}}\right) / 2 ; E_{\mathrm{pa}}$ and $E_{\mathrm{pc}}$ are the peak anodic and peak cathodic potentials, respectively.

[c] $\Delta E_{\mathrm{p}}=\left(E_{\mathrm{pa}}-E_{\mathrm{pc}}\right)$.

${ }^{\text {[d] }} E_{\mathrm{pa}}$ denotes the anodic peak potential for the irreversible oxidations.

[e] $E_{\mathrm{pc}}$ denotes the cathodic peak potential for the irreversible reductions.

[f] $E_{\mathrm{HOMO}}$ and $E_{\mathrm{LUMO}}$ were calculated from electrochemical potentials with the formula, $E_{\mathrm{HOMO}}=-\mathrm{e}\left(4.8 \mathrm{~V}+E_{1 / 2}{ }^{\mathrm{ox}}\right) ; E_{\mathrm{LUMO}}=-\mathrm{e}\left(4.8 \mathrm{~V}+E_{1 / 2}{ }^{\mathrm{red}}\right)$. 


\section{Computational Details}

The density functional theory (DFT) and time-dependent density functional theory (TDDFT) ${ }^{9}$ calculations in this work were performed using the Gaussian 09 suite of programs. ${ }^{10}$ The geometries of the ground state and the lowest-lying triplet excited state of 1, 2 and 5 were fully optimized in dichloromethane by DFT using the hybrid Perdew, Burke, and Ernzerhof (PBE0) functional, ${ }^{11}$ in conjunction with the conductor-like polarizable continuum model (CPCM).${ }^{12}$ The triplet excited states were optimized with the unrestricted UPBE0/CPCM method. Vibrational frequency calculations were then carried out on the optimized geometries to confirm that they are minima $(\mathrm{NIMAG}=0$ ) on the potential energy surface $(\mathrm{PES})$. On the basis of the optimized groundstate geometries, TDDFT calculations at the same level of theory with CPCM were performed in order to compute the singlet-singlet transitions in the electronic absorption spectra. The Cartesian coordinates of the optimized geometries of $\mathbf{1}, \mathbf{2}$ and $\mathbf{5}$ are given in Tables S10-S15. The Stuttgart effective core potentials (ECPs) and the associated basis set were used to describe $\mathrm{Au}^{13}$ with f-type polarization functions $(\zeta=1.050),{ }^{14}$ whereas the $6-31 \mathrm{G}(\mathrm{d}, \mathrm{p})$ basis set ${ }^{15}$ was applied for all other atoms. All the DFT and TDDFT calculations were performed with a pruned $(99,590)$ grid for numerical integration. 
Table S9 List of first fifteen singlet excited states $\left(S_{n}\right)$ of $\mathbf{1}, \mathbf{2}$ and $\mathbf{5}$ computed by TDDFT/CPCM using dichloromethane as the solvent.

\begin{tabular}{|c|c|c|c|c|}
\hline Complex & $\overline{S_{n}}$ & $\begin{array}{c}\text { Excitation }^{[\mathrm{a}]} \\
{\text { (Coefficient })^{[\mathrm{b}]}}^{\text {Con }}\end{array}$ & $\begin{array}{l}\text { Vertical excitation } \\
\text { wavelength / nm }\end{array}$ & $\mathrm{f}^{[\mathrm{c}]}$ \\
\hline \multirow[t]{23}{*}{1} & $\mathrm{~S}_{1}$ & $\mathrm{H} \rightarrow \mathrm{L}(0.70)$ & 488 & 0.101 \\
\hline & $\mathrm{S}_{2}$ & $\mathrm{H} \rightarrow \mathrm{L}+1(0.69)$ & 399 & 0.118 \\
\hline & $\mathrm{S}_{3}$ & $\mathrm{H}-1 \rightarrow \mathrm{L}(0.57)$ & 388 & 0.063 \\
\hline & & $\mathrm{H}-2 \rightarrow \mathrm{L}(-0.38)$ & & \\
\hline & $\mathrm{S}_{4}$ & $\mathrm{H}-2 \rightarrow \mathrm{L}(0.52)$ & 381 & 0.048 \\
\hline & & $\mathrm{H}-1 \rightarrow \mathrm{L}(0.41)$ & & \\
\hline & $\mathrm{S}_{5}$ & $\mathrm{H} \rightarrow \mathrm{L}+3(0.63)$ & 371 & 0.006 \\
\hline & $\mathrm{S}_{6}$ & $\mathrm{H} \rightarrow \mathrm{L}+2(0.57)$ & 349 & 0.121 \\
\hline & $\mathrm{S}_{7}$ & $\mathrm{H}-3 \rightarrow \mathrm{L}(0.59)$ & 341 & 0.102 \\
\hline & $\mathrm{S}_{8}$ & $\mathrm{H}-4 \rightarrow \mathrm{L}(0.56)$ & 327 & 0.010 \\
\hline & & $\mathrm{H}-1 \rightarrow \mathrm{L}+1(0.31)$ & & \\
\hline & $\mathrm{S}_{9}$ & $\mathrm{H}-1 \rightarrow \mathrm{L}+1(0.62)$ & 326 & 0.050 \\
\hline & $S_{10}$ & $\mathrm{H}-2 \rightarrow \mathrm{L}+1(0.51)$ & 306 & 0.028 \\
\hline & & $\mathrm{H}-6 \rightarrow \mathrm{L}(0.31)$ & & \\
\hline & $\mathrm{S}_{11}$ & $\mathrm{H}-5 \rightarrow \mathrm{L}(0.55)$ & 303 & 0.684 \\
\hline & & $\mathrm{H}-2 \rightarrow \mathrm{L}+1(0.32)$ & & \\
\hline & $S_{12}$ & $\mathrm{H} \rightarrow \mathrm{L}+5(0.40)$ & 302 & 0.049 \\
\hline & & $\mathrm{H} \rightarrow \mathrm{L}+4(-0.38)$ & & \\
\hline & $S_{13}$ & $\mathrm{H}-1 \rightarrow \mathrm{L}+3(0.59)$ & 298 & 0.016 \\
\hline & $\mathrm{S}_{14}$ & $\mathrm{H}-6 \rightarrow \mathrm{L}(0.41)$ & 297 & 0.119 \\
\hline & & $\mathrm{H}-3 \rightarrow \mathrm{L}+1(-0.33)$ & & \\
\hline & $S_{15}$ & $\mathrm{H}-3 \rightarrow \mathrm{L}+1(0.46)$ & 295 & 0.172 \\
\hline & & $\mathrm{H}-6 \rightarrow \mathrm{L}(0.31)$ & & \\
\hline \multirow[t]{12}{*}{2} & $\mathrm{~S}_{1}$ & $\mathrm{H} \rightarrow \mathrm{L}(0.70)$ & 441 & 0.117 \\
\hline & $\mathrm{S}_{2}$ & $\mathrm{H}-2 \rightarrow \mathrm{L}(0.69)$ & 380 & 0.103 \\
\hline & $\mathrm{S}_{3}$ & $\mathrm{H} \rightarrow \mathrm{L}+1(0.70)$ & 358 & 0.065 \\
\hline & $\mathrm{S}_{4}$ & $\mathrm{H}-1 \rightarrow \mathrm{L}(0.69)$ & 356 & 0.006 \\
\hline & $\mathrm{S}_{5}$ & $\mathrm{H}-3 \rightarrow \mathrm{L}(0.60)$ & 327 & 0.008 \\
\hline & $\mathrm{S}_{6}$ & $\mathrm{H} \rightarrow \mathrm{L}+2(0.67)$ & 323 & 0.148 \\
\hline & $S_{7}$ & $\mathrm{H}-4 \rightarrow \mathrm{L}(0.50)$ & 304 & 0.015 \\
\hline & & $\mathrm{H}-2 \rightarrow \mathrm{L}+1(0.34)$ & & \\
\hline & $\mathrm{S}_{8}$ & $\mathrm{H}-1 \rightarrow \mathrm{L}+1(0.50)$ & 301 & 0.409 \\
\hline & & $\mathrm{H}-4 \rightarrow \mathrm{L}(0.35)$ & & \\
\hline & $\mathrm{S}_{9}$ & $\mathrm{H}-1 \rightarrow \mathrm{L}+1(0.46)$ & 300 & 0.175 \\
\hline & & $\mathrm{H}-2 \rightarrow \mathrm{L}+1(0.37)$ & & \\
\hline
\end{tabular}




\begin{tabular}{|c|c|c|c|c|}
\hline & $S_{10}$ & $\mathrm{H}-5 \rightarrow \mathrm{L}(0.63)$ & 298 & 0.439 \\
\hline & $S_{11}$ & $\mathrm{H} \rightarrow \mathrm{L}+3(0.40)$ & 295 & 0.038 \\
\hline & & $\mathrm{H} \rightarrow \mathrm{L}+4(0.37)$ & & \\
\hline & $\mathrm{S}_{12}$ & $\mathrm{H}-6 \rightarrow \mathrm{L}(0.38)$ & 293 & 0.090 \\
\hline & $S_{13}$ & $\mathrm{H}-7 \rightarrow \mathrm{L}(0.53)$ & 291 & 0.032 \\
\hline & & $\mathrm{H}-6 \rightarrow \mathrm{L}(0.31)$ & & \\
\hline & $\mathrm{S}_{14}$ & $\mathrm{H}-3 \rightarrow \mathrm{L}+1(0.49)$ & 279 & 0.165 \\
\hline & $\mathrm{S}_{15}$ & $\mathrm{H} \rightarrow \mathrm{L}+6(0.40)$ & 279 & 0.046 \\
\hline & & $\mathrm{H}-3 \rightarrow \mathrm{L}+1(-0.31)$ & & \\
\hline \multirow[t]{21}{*}{5} & $\mathrm{~S}_{1}$ & $\mathrm{H} \rightarrow \mathrm{L}(0.70)$ & 532 & 0.018 \\
\hline & $\mathrm{S}_{2}$ & $\mathrm{H} \rightarrow \mathrm{L}+1(0.70)$ & 407 & 0.000 \\
\hline & $\mathrm{S}_{3}$ & $\mathrm{H}-1 \rightarrow \mathrm{L}(0.70)$ & 405 & 0.000 \\
\hline & $\mathrm{S}_{4}$ & $\mathrm{H}-2 \rightarrow \mathrm{L}(0.67)$ & 389 & 0.093 \\
\hline & $\mathrm{S}_{5}$ & $\mathrm{H} \rightarrow \mathrm{L}+2(0.67)$ & 368 & 0.048 \\
\hline & $\mathrm{S}_{6}$ & $\mathrm{H}-4 \rightarrow \mathrm{L}(0.40)$ & 331 & 0.037 \\
\hline & & $\mathrm{H}-5 \rightarrow \mathrm{L}(0.39)$ & & \\
\hline & & $\mathrm{H} \rightarrow \mathrm{L}+3(-0.35)$ & & \\
\hline & $\mathrm{S}_{7}$ & $\mathrm{H} \rightarrow \mathrm{L}+3(0.54)$ & 330 & 0.027 \\
\hline & $\mathrm{S}_{8}$ & $\mathrm{H}-1 \rightarrow \mathrm{L}+1(0.68)$ & 330 & 0.005 \\
\hline & $\mathrm{S}_{9}$ & $\mathrm{H}-3 \rightarrow \mathrm{L}(0.50)$ & 320 & 0.120 \\
\hline & $S_{10}$ & $\mathrm{H}-5 \rightarrow \mathrm{L}(0.44)$ & 308 & 0.301 \\
\hline & & $\mathrm{H}-6 \rightarrow \mathrm{L}(0.37)$ & & \\
\hline & $S_{11}$ & $\mathrm{H} \rightarrow \mathrm{L}+4(0.53)$ & 305 & 0.018 \\
\hline & & $\mathrm{H} \rightarrow \mathrm{L}+5(0.38)$ & & \\
\hline & $S_{12}$ & $\mathrm{H}-6 \rightarrow \mathrm{L}(0.45)$ & 302 & 0.056 \\
\hline & & $\mathrm{H}-3 \rightarrow \mathrm{L}(-0.44)$ & & \\
\hline & $\mathrm{S}_{13}$ & $\mathrm{H}-2 \rightarrow \mathrm{L}+1(0.59)$ & 300 & 0.634 \\
\hline & & $\mathrm{H}-8 \rightarrow \mathrm{L}(0.32)$ & & \\
\hline & $\mathrm{S}_{14}$ & $\mathrm{H}-7 \rightarrow \mathrm{L}(0.66)$ & 298 & 0.010 \\
\hline & $S_{15}$ & $\mathrm{H}-1 \rightarrow \mathrm{L}+2(0.70)$ & 295 & 0.000 \\
\hline
\end{tabular}

[a] Orbitals involved in the major excitation $(\mathrm{H}=\mathrm{HOMO}$ and $\mathrm{L}=\mathrm{LUMO})$.

${ }^{[b]}$ The coefficients in the configuration interaction (CI) expansion.

${ }^{[c]}$ Oscillator strengths. 
Table S10 Cartesian coordinates of the optimized ground-state geometry of $\mathbf{1}$.

\begin{tabular}{|c|c|c|c|c|c|c|c|c|c|}
\hline 1 & $\mathrm{C}$ & -0.000081 & 1.963459 & 0.412740 & 57 & $\mathrm{C}$ & 3.313954 & -3.893826 & 0.488386 \\
\hline 2 & $\mathrm{C}$ & -1.397833 & 2.245457 & 0.440960 & 58 & $\mathrm{C}$ & 5.206241 & -2.455395 & 0.187281 \\
\hline 3 & $\mathrm{C}$ & 0.858172 & 2.995180 & 0.779023 & 59 & $\mathrm{C}$ & 4.301910 & 2.517033 & -1.482737 \\
\hline 4 & $\mathrm{C}$ & -1.859727 & 3.526014 & 0.743327 & 60 & $\mathrm{H}$ & 2.168656 & 2.242916 & -1.375054 \\
\hline 5 & C & 0.412344 & 4.288819 & 1.112378 & 61 & $\mathrm{C}$ & 5.702756 & 0.691101 & -0.794107 \\
\hline 6 & $\mathrm{H}$ & 1.925928 & 2.797644 & 0.806382 & 62 & $\mathrm{C}$ & 4.704050 & -3.721358 & 0.500139 \\
\hline 7 & $\mathrm{C}$ & -0.961754 & 4.538705 & 1.064028 & 63 & $\mathrm{H}$ & 2.923820 & -4.879900 & 0.707342 \\
\hline 8 & $\mathrm{H}$ & -2.925660 & 3.739229 & 0.755990 & 64 & $\mathrm{H}$ & 6.278520 & -2.280267 & 0.143676 \\
\hline 9 & $\mathrm{H}$ & -1.351025 & 5.523002 & 1.299253 & 65 & $\mathrm{C}$ & 5.598392 & 1.990037 & -1.284381 \\
\hline 10 & $\mathrm{C}$ & -2.299212 & 1.108783 & 0.235309 & 66 & $\mathrm{H}$ & 4.193961 & 3.511292 & -1.908691 \\
\hline 11 & $\mathrm{C}$ & -3.688205 & 1.073061 & 0.145307 & 67 & $\mathrm{H}$ & 6.674511 & 0.225271 & -0.662786 \\
\hline 12 & $\mathrm{C}$ & -4.371858 & -0.137990 & -0.061993 & 68 & $\mathrm{~N}$ & 2.286430 & -0.387480 & -0.294717 \\
\hline 13 & $\mathrm{H}$ & -4.253764 & 1.996722 & 0.193162 & 69 & $\mathrm{C}$ & 5.672718 & -4.866050 & 0.817218 \\
\hline 14 & $\mathrm{~N}$ & -1.644265 & -0.063706 & 0.152432 & 70 & $\mathrm{C}$ & 4.948333 & -6.176318 & 1.138570 \\
\hline 15 & $\mathrm{C}$ & -5.846765 & -0.096031 & -0.179260 & 71 & $\mathrm{H}$ & 4.294266 & -6.076892 & 2.011530 \\
\hline 16 & $\mathrm{C}$ & -6.605081 & 0.622550 & 0.749858 & 72 & $\mathrm{H}$ & 4.345127 & -6.527106 & 0.294270 \\
\hline 17 & $\mathrm{C}$ & -6.523497 & -0.733337 & -1.227817 & 73 & $\mathrm{H}$ & 5.684663 & -6.954758 & 1.363798 \\
\hline 18 & $\mathrm{C}$ & -7.991430 & 0.690286 & 0.644056 & 74 & $\mathrm{C}$ & 6.531955 & -4.484328 & 2.033942 \\
\hline 19 & $\mathrm{H}$ & -6.108104 & 1.118242 & 1.579638 & 75 & $\mathrm{H}$ & 7.240045 & -5.287587 & 2.267955 \\
\hline 20 & $\mathrm{C}$ & -7.905085 & -0.650271 & -1.332802 & 76 & $\mathrm{H}$ & 7.109346 & -3.572720 & 1.851672 \\
\hline 21 & $\mathrm{H}$ & -5.964135 & -1.273325 & -1.985810 & 77 & $\mathrm{H}$ & 5.905514 & -4.314919 & 2.916174 \\
\hline 22 & $\mathrm{C}$ & -8.675532 & 0.057597 & -0.398578 & 78 & $\mathrm{C}$ & 6.589758 & -5.111129 & -0.392523 \\
\hline 23 & $\mathrm{H}$ & -8.538037 & 1.248437 & 1.396322 & 79 & $\mathrm{H}$ & 6.005028 & -5.394803 & -1.274008 \\
\hline 24 & $\mathrm{H}$ & -8.390380 & -1.148114 & -2.167690 & 80 & $\mathrm{H}$ & 7.171650 & -4.220083 & -0.647521 \\
\hline 25 & $\mathrm{C}$ & -10.196600 & 0.113086 & -0.549345 & 81 & $\mathrm{H}$ & 7.296318 & -5.920915 & -0.177122 \\
\hline 26 & $\mathrm{C}$ & -10.860249 & 0.927118 & 0.564478 & 82 & $\mathrm{C}$ & 6.818631 & 2.845088 & -1.637077 \\
\hline 27 & $\mathrm{H}$ & -10.667925 & 0.497406 & 1.553235 & 83 & $\mathrm{C}$ & 8.135631 & 2.115063 & -1.360287 \\
\hline 28 & $\mathrm{H}$ & -10.518569 & 1.967567 & 0.567998 & 84 & $\mathrm{H}$ & 8.234382 & 1.842624 & -0.304084 \\
\hline 29 & $\mathrm{H}$ & -11.944229 & 0.935626 & 0.412714 & 85 & $\mathrm{H}$ & 8.229292 & 1.203570 & -1.960239 \\
\hline 30 & $\mathrm{C}$ & -10.762490 & -1.316227 & -0.507298 & 86 & $\mathrm{H}$ & 8.977330 & 2.767286 & -1.615372 \\
\hline 31 & $\mathrm{H}$ & -11.851816 & -1.292382 & -0.621498 & 87 & $\mathrm{C}$ & 6.799734 & 4.133319 & -0.797298 \\
\hline 32 & $\mathrm{H}$ & -10.356391 & -1.938803 & -1.310283 & 88 & $\mathrm{H}$ & 5.894669 & 4.721953 & -0.976997 \\
\hline 33 & $\mathrm{H}$ & -10.530822 & -1.801061 & 0.446781 & 89 & $\mathrm{H}$ & 6.843332 & 3.901859 & 0.272306 \\
\hline 34 & $\mathrm{C}$ & -10.549336 & 0.761477 & -1.898504 & 90 & $\mathrm{H}$ & 7.662441 & 4.762394 & -1.045134 \\
\hline 35 & $\mathrm{H}$ & -11.637080 & 0.801112 & -2.022885 & 91 & $\mathrm{C}$ & 6.779524 & 3.213121 & -3.129641 \\
\hline 36 & $\mathrm{H}$ & -10.162828 & 1.784454 & -1.954068 & 92 & $\mathrm{H}$ & 6.801922 & 2.313340 & -3.753536 \\
\hline 37 & $\mathrm{H}$ & -10.137593 & 0.198301 & -2.741457 & 93 & $\mathrm{H}$ & 5.878136 & 3.778124 & -3.386136 \\
\hline 38 & C & 1.430732 & 5.358962 & 1.511553 & 94 & $\mathrm{H}$ & 7.645978 & 3.831129 & -3.391713 \\
\hline 39 & C & 0.767920 & 6.700137 & 1.837922 & 95 & $\mathrm{C}$ & -3.639655 & -1.341397 & -0.143268 \\
\hline 40 & $\mathrm{H}$ & 1.536666 & 7.427603 & 2.117628 & 96 & C & -2.257329 & -1.248912 & -0.011162 \\
\hline 41 & $\mathrm{H}$ & 0.225404 & 7.106530 & 0.977730 & 97 & $\mathrm{Au}$ & 0.340887 & -0.089332 & 0.048018 \\
\hline 42 & $\mathrm{H}$ & 0.069881 & 6.616670 & 2.677664 & 98 & $\mathrm{C}$ & 0.026159 & -2.107705 & -0.002295 \\
\hline 43 & $\mathrm{C}$ & 2.420520 & 5.578864 & 0.355162 & 99 & $\mathrm{C}$ & -1.356002 & -2.387617 & -0.020730 \\
\hline 4 & $\mathrm{H}$ & 2.965311 & 4.663395 & 0.105633 & 100 & C & 0.944157 & -3.151115 & 0.119264 \\
\hline 45 & $\mathrm{H}$ & 1.899480 & 5.919796 & -0.545872 & 101 & $\mathrm{C}$ & -1.875206 & -3.677094 & 0.074256 \\
\hline 46 & $\mathrm{H}$ & 3.156689 & 6.342221 & 0.631328 & 102 & $\mathrm{C}$ & 0.416990 & -4.464612 & 0.145806 \\
\hline 47 & $\mathrm{C}$ & 2.200025 & 4.885792 & 2.756555 & 103 & $\mathrm{C}$ & -0.948843 & -4.722023 & 0.131943 \\
\hline 48 & $\mathrm{H}$ & 1.519759 & 4.728117 & 3.600127 & 104 & $\mathrm{H}$ & 1.088928 & -5.316389 & 0.180652 \\
\hline 49 & $\mathrm{H}$ & 2.734465 & 3.948928 & 2.572879 & 105 & $\mathrm{H}$ & -1.296503 & -5.750745 & 0.183362 \\
\hline 50 & $\mathrm{H}$ & 2.938434 & 5.639892 & 3.050942 & 106 & $\mathrm{C}$ & -3.364835 & -3.842978 & 0.238774 \\
\hline 51 & $\mathrm{C}$ & 2.923342 & -1.586300 & -0.019134 & 107 & $\mathrm{H}$ & -3.689931 & -4.808402 & -0.161442 \\
\hline 52 & $\mathrm{C}$ & 3.268174 & 0.531027 & -0.637033 & 108 & $\mathrm{H}$ & -3.582245 & -3.870046 & 1.316390 \\
\hline 53 & $\mathrm{C}$ & 2.389854 & -2.874009 & 0.211140 & 109 & $\mathrm{C}$ & -4.189279 & -2.722554 & -0.410869 \\
\hline 54 & $\mathrm{C}$ & 4.333195 & -1.406267 & -0.077954 & 110 & $\mathrm{H}$ & -4.202204 & -2.879305 & -1.498855 \\
\hline 55 & C & 3.145201 & 1.814013 & -1.177725 & 111 & $\mathrm{H}$ & -5.228487 & -2.790584 & -0.078385 \\
\hline 56 & $\mathrm{C}$ & 4.550961 & -0.041643 & -0.486444 & & & & & \\
\hline
\end{tabular}


Table S11 Cartesian coordinates of the optimized ground-state geometry of 2.

\begin{tabular}{|c|c|c|c|c|c|c|c|c|c|}
\hline 1 & $\mathrm{C}$ & -0.568911 & 2.431867 & -0.129108 & 57 & $\mathrm{C}$ & 6.377793 & -2.086873 & -0.162320 \\
\hline 2 & $\mathrm{C}$ & -1.998938 & 2.374641 & -0.140328 & 58 & $\mathrm{C}$ & 2.911636 & 1.872678 & 0.515781 \\
\hline 3 & $\mathrm{C}$ & -0.000562 & 3.685739 & -0.336234 & 59 & $\mathrm{C}$ & 5.102928 & 0.899848 & 0.445254 \\
\hline 4 & $\mathrm{C}$ & -2.757679 & 3.532162 & -0.304364 & 60 & C & 6.646802 & -3.419301 & -0.484983 \\
\hline 5 & $\mathrm{C}$ & -0.748170 & 4.866885 & -0.513447 & 61 & $\mathrm{H}$ & 5.717489 & -5.303898 & -0.977134 \\
\hline 6 & $\mathrm{H}$ & 1.081525 & 3.766586 & -0.380224 & 62 & $\mathrm{H}$ & 7.190902 & -1.376135 & -0.037629 \\
\hline 7 & $\mathrm{C}$ & -2.139981 & 4.765709 & -0.480960 & 63 & C & 4.308700 & 2.028256 & 0.625777 \\
\hline 8 & $\mathrm{H}$ & -3.843556 & 3.480802 & -0.308156 & 64 & $\mathrm{H}$ & 2.291345 & 2.752815 & 0.638044 \\
\hline 9 & $\mathrm{H}$ & -2.760857 & 5.645537 & -0.607479 & 65 & $\mathrm{H}$ & 6.185323 & 0.963419 & 0.484773 \\
\hline 10 & $\mathrm{C}$ & -2.624026 & 1.049357 & -0.019879 & 66 & $\mathrm{~N}$ & 2.783803 & -1.831461 & 0.021623 \\
\hline 11 & $\mathrm{C}$ & -3.971205 & 0.691459 & -0.004492 & 67 & C & 8.097219 & -3.889358 & -0.632698 \\
\hline 12 & $\mathrm{C}$ & -4.354198 & -0.660748 & 0.045538 & 68 & C & 8.193735 & -5.375705 & -0.987755 \\
\hline 13 & $\mathrm{H}$ & -4.739669 & 1.453116 & -0.074403 & 69 & $\mathrm{H}$ & 7.736568 & -6.008217 & -0.219394 \\
\hline 14 & $\mathrm{~N}$ & -1.722730 & 0.062057 & 0.057790 & 70 & $\mathrm{H}$ & 7.714079 & -5.597954 & -1.946901 \\
\hline 15 & $\mathrm{C}$ & -5.801130 & -0.976233 & 0.015807 & 71 & $\mathrm{H}$ & 9.246819 & -5.663470 & -1.070652 \\
\hline 16 & $\mathrm{C}$ & -6.685577 & -0.300260 & 0.861395 & 72 & C & 8.842432 & -3.663057 & 0.693082 \\
\hline 17 & $\mathrm{C}$ & -6.333899 & -1.920061 & -0.872148 & 73 & $\mathrm{H}$ & 9.886023 & -3.984949 & 0.601866 \\
\hline 18 & $\mathrm{C}$ & -8.051725 & -0.566610 & 0.830882 & 74 & $\mathrm{H}$ & 8.842503 & -2.607767 & 0.982933 \\
\hline 19 & $\mathrm{H}$ & -6.299511 & 0.430149 & 1.567631 & 75 & $\mathrm{H}$ & 8.379323 & -4.235010 & 1.504064 \\
\hline 20 & $\mathrm{C}$ & -7.698865 & -2.170942 & -0.904698 & 76 & $\mathrm{C}$ & 8.787231 & -3.084054 & -1.746118 \\
\hline 21 & $\mathrm{H}$ & -5.680642 & -2.441919 & -1.564976 & 77 & $\mathrm{H}$ & 8.280600 & -3.231216 & -2.705750 \\
\hline 22 & $\mathrm{C}$ & -8.592557 & -1.506177 & -0.052256 & 78 & $\mathrm{H}$ & 8.791696 & -2.011581 & -1.528674 \\
\hline 23 & $\mathrm{H}$ & -8.696558 & -0.025858 & 1.515052 & 79 & $\mathrm{H}$ & 9.828710 & -3.406395 & -1.857635 \\
\hline 24 & $\mathrm{H}$ & -8.072702 & -2.901032 & -1.617244 & 80 & C & 4.898133 & 3.413870 & 0.906686 \\
\hline 25 & $\mathrm{C}$ & -10.088092 & -1.820332 & -0.118993 & 81 & C & 6.420329 & 3.369328 & 1.070625 \\
\hline 26 & $\mathrm{C}$ & -10.897767 & -1.003355 & 0.891330 & 82 & $\mathrm{H}$ & 6.720156 & 2.713665 & 1.895075 \\
\hline 27 & $\mathrm{H}$ & -10.588773 & -1.207915 & 1.921855 & 83 & $\mathrm{H}$ & 6.919273 & 3.025660 & 0.158388 \\
\hline 28 & $\mathrm{H}$ & -10.807180 & 0.073044 & 0.711284 & 84 & $\mathrm{H}$ & 6.793822 & 4.374548 & 1.291303 \\
\hline 29 & $\mathrm{H}$ & -11.957210 & -1.264875 & 0.805447 & 85 & C & 4.297352 & 3.980295 & 2.203547 \\
\hline 30 & $\mathrm{C}$ & -10.307783 & -3.312882 & 0.178980 & 86 & $\mathrm{H}$ & 3.209379 & 4.081926 & 2.142843 \\
\hline 31 & $\mathrm{H}$ & -11.375555 & -3.552805 & 0.128329 & 87 & $\mathrm{H}$ & 4.526499 & 3.331137 & 3.055291 \\
\hline 32 & $\mathrm{H}$ & -9.787778 & -3.952842 & -0.540283 & 88 & $\mathrm{H}$ & 4.712625 & 4.972999 & 2.410707 \\
\hline 33 & $\mathrm{H}$ & -9.948569 & -3.569033 & 1.181152 & 89 & $\mathrm{C}$ & 4.572116 & 4.356394 & -0.263244 \\
\hline 34 & $\mathrm{C}$ & -10.610463 & -1.500186 & -1.529597 & 90 & $\mathrm{H}$ & 5.005506 & 3.983525 & -1.197245 \\
\hline 35 & $\mathrm{H}$ & -11.680390 & -1.726760 & -1.594472 & 91 & $\mathrm{H}$ & 3.492829 & 4.457420 & -0.413006 \\
\hline 36 & $\mathrm{H}$ & -10.471938 & -0.440261 & -1.767006 & 92 & $\mathrm{H}$ & 4.977748 & 5.356650 & -0.073355 \\
\hline 37 & $\mathrm{H}$ & -10.097750 & -2.088202 & -2.296842 & 93 & C & -3.364415 & -1.663545 & 0.132854 \\
\hline 38 & $\mathrm{C}$ & -0.027820 & 6.199282 & -0.733881 & 94 & C & -2.040129 & -1.233279 & 0.171081 \\
\hline 39 & $\mathrm{C}$ & -1.004402 & 7.361617 & -0.936152 & 95 & $\mathrm{Au}$ & 0.265321 & 0.496785 & 0.074754 \\
\hline 40 & $\mathrm{H}$ & -0.441015 & 8.286662 & -1.095208 & 96 & C & 0.391080 & -1.536378 & 0.179014 \\
\hline 41 & $\mathrm{H}$ & -1.644215 & 7.208284 & -1.811584 & 97 & C & -0.889694 & -2.118866 & 0.304896 \\
\hline 42 & $\mathrm{H}$ & -1.646381 & 7.512021 & -0.061820 & 98 & C & 1.504086 & -2.373011 & 0.226635 \\
\hline 43 & $\mathrm{C}$ & 0.861288 & 6.099960 & -1.984789 & 99 & C & -1.099313 & -3.467601 & 0.592310 \\
\hline 44 & $\mathrm{H}$ & 1.614350 & 5.311608 & -1.892603 & 100 & C & 1.312036 & -3.733885 & 0.542431 \\
\hline 45 & $\mathrm{H}$ & 0.259875 & 5.888011 & -2.875054 & 101 & C & 0.038903 & -4.262602 & 0.727228 \\
\hline 46 & $\mathrm{H}$ & 1.388382 & 7.046621 & -2.147373 & 102 & $\mathrm{H}$ & 2.163800 & -4.382099 & 0.700275 \\
\hline 47 & $\mathrm{C}$ & 0.844297 & 6.516143 & 0.492518 & 103 & $\mathrm{H}$ & -0.059538 & -5.311124 & 0.996291 \\
\hline 48 & $\mathrm{H}$ & 0.231476 & 6.601148 & 1.396056 & 104 & C & -2.503466 & -3.934168 & 0.888609 \\
\hline 49 & $\mathrm{H}$ & 1.597661 & 5.742839 & 0.668892 & 105 & $\mathrm{H}$ & -2.606808 & -5.001523 & 0.670001 \\
\hline 50 & $\mathrm{H}$ & 1.369698 & 7.466475 & 0.346310 & 106 & $\mathrm{H}$ & -2.673732 & -3.825129 & 1.969497 \\
\hline 51 & $\mathrm{C}$ & 3.980442 & -2.536651 & -0.165295 & 107 & $\mathrm{C}$ & -3.588781 & -3.156413 & 0.132645 \\
\hline 52 & $\mathrm{C}$ & 3.098536 & -0.470981 & 0.179946 & 108 & $\mathrm{H}$ & -3.604742 & -3.497267 & -0.912540 \\
\hline 53 & $\mathrm{C}$ & 5.065355 & -1.645093 & -0.016526 & 109 & $\mathrm{H}$ & -4.571458 & -3.396728 & 0.547644 \\
\hline 54 & $\mathrm{C}$ & 2.263669 & 0.658812 & 0.267302 & 110 & C & 4.227436 & -3.857885 & -0.539719 \\
\hline 55 & $\mathrm{C}$ & 4.503449 & -0.337520 & 0.219883 & 111 & $\mathrm{H}$ & 3.431729 & -4.557785 & -0.760296 \\
\hline & C & 5.549389 & -4.273135 & .684966 & & & & & \\
\hline
\end{tabular}


Table S12 Cartesian coordinates of the optimized ground-state geometry of 5 .

\begin{tabular}{|c|c|c|c|c|c|c|c|c|c|}
\hline 1 & C & -0.074670 & 1.500160 & -0.790295 & 58 & C & -3.718305 & 2.100783 & 2.523814 \\
\hline 2 & C & 1.296024 & 1.828975 & -0.978942 & 59 & $\mathrm{H}$ & -1.667508 & 1.667330 & 2.020139 \\
\hline 3 & $\mathrm{C}$ & -1.037479 & 2.413114 & -1.192493 & 60 & $\mathrm{C}$ & -5.438192 & 0.787895 & 1.470598 \\
\hline 4 & C & 1.644019 & 3.049420 & -1.555663 & 61 & C & -5.199236 & -2.753121 & -1.688813 \\
\hline 5 & C & -0.703780 & 3.652617 & -1.771478 & 62 & $\mathrm{H}$ & -3.586283 & -3.765849 & -2.711264 \\
\hline 6 & $\mathrm{H}$ & -2.084146 & 2.161187 & -1.041848 & 63 & $\mathrm{H}$ & -6.528441 & -1.528511 & -0.507922 \\
\hline 7 & C & 0.652347 & 3.946800 & -1.945122 & 64 & $\mathrm{C}$ & -5.092317 & 1.811171 & 2.349852 \\
\hline 8 & $\mathrm{H}$ & 2.687505 & 3.314008 & -1.707375 & 65 & $\mathrm{H}$ & -3.427952 & 2.893593 & 3.208583 \\
\hline 9 & $\mathrm{H}$ & 0.954692 & 4.888799 & -2.389016 & 66 & $\mathrm{H}$ & -6.479919 & 0.528701 & 1.307593 \\
\hline 10 & C & 2.269983 & 0.826214 & -0.531728 & 67 & $\mathrm{~N}$ & -2.284042 & -0.421970 & 0.199474 \\
\hline 11 & $\mathrm{C}$ & 3.659597 & 0.875040 & -0.534151 & 68 & $\mathrm{C}$ & -6.340133 & -3.552981 & -2.329037 \\
\hline 12 & $\mathrm{C}$ & 4.421212 & -0.172761 & 0.014445 & 69 & $\mathrm{C}$ & -5.830617 & -4.617724 & -3.304796 \\
\hline 13 & $\mathrm{H}$ & 4.166852 & 1.752626 & -0.918548 & 70 & $\mathrm{H}$ & -5.270710 & -4.174647 & -4.135283 \\
\hline 14 & $\mathrm{~N}$ & 1.691297 & -0.285809 & -0.033581 & 71 & $\mathrm{H}$ & -5.185298 & -5.349985 & -2.807854 \\
\hline 15 & C & 5.895063 & -0.033097 & 0.014700 & 72 & $\mathrm{H}$ & -6.680787 & -5.160617 & -3.731005 \\
\hline 16 & C & 6.561245 & 0.357022 & -1.150897 & 73 & $\mathrm{C}$ & -7.265334 & -2.600005 & -3.103966 \\
\hline 17 & C & 6.659621 & -0.244734 & 1.169646 & 74 & $\mathrm{H}$ & -8.092098 & -3.156567 & -3.560593 \\
\hline 18 & C & 7.944138 & 0.514928 & -1.166932 & 75 & $\mathrm{H}$ & -7.697699 & -1.835864 & -2.450604 \\
\hline 19 & $\mathrm{H}$ & 5.994593 & 0.519216 & -2.064053 & 76 & $\mathrm{H}$ & -6.717417 & -2.088517 & -3.902555 \\
\hline 20 & C & 8.036713 & -0.071589 & 1.148215 & 77 & C & -7.151197 & -4.261684 & -1.231632 \\
\hline 21 & $\mathrm{H}$ & 6.172504 & -0.516796 & 2.101069 & 78 & $\mathrm{H}$ & -6.520276 & -4.957975 & -0.668884 \\
\hline 22 & C & 8.715717 & 0.306168 & -0.019563 & 79 & $\mathrm{H}$ & -7.580529 & -3.548433 & -0.521288 \\
\hline 23 & $\mathrm{H}$ & 8.417879 & 0.805141 & -2.098338 & 80 & $\mathrm{H}$ & -7.976838 & -4.831343 & -1.673852 \\
\hline 24 & $\mathrm{H}$ & 8.591423 & -0.232389 & 2.068466 & 81 & $\mathrm{C}$ & -6.132396 & 2.624529 & 3.126139 \\
\hline 25 & C & 10.235658 & 0.475531 & 0.002171 & 82 & C & -7.564149 & 2.179085 & 2.816477 \\
\hline 26 & C & 10.793185 & 0.882694 & -1.364222 & 83 & $\mathrm{H}$ & -7.807268 & 2.301638 & 1.755613 \\
\hline 27 & $\mathrm{H}$ & 10.581363 & 0.130426 & -2.131375 & 84 & $\mathrm{H}$ & -7.731179 & 1.131192 & 3.087742 \\
\hline 28 & $\mathrm{H}$ & 10.387268 & 1.842528 & -1.700690 & 85 & $\mathrm{H}$ & -8.270436 & 2.787238 & 3.391277 \\
\hline 29 & $\mathrm{H}$ & 11.880470 & 0.989084 & -1.296564 & 86 & C & -6.003886 & 4.111612 & 2.755735 \\
\hline 30 & C & 10.888571 & -0.854397 & 0.414420 & 87 & $\mathrm{H}$ & -5.010425 & 4.504689 & 2.992957 \\
\hline 31 & $\mathrm{H}$ & 11.978318 & -0.745082 & 0.441432 & 88 & $\mathrm{H}$ & -6.178252 & 4.262633 & 1.685001 \\
\hline 32 & $\mathrm{H}$ & 10.561284 & -1.178778 & 1.406873 & 89 & $\mathrm{H}$ & -6.739372 & 4.707829 & 3.308219 \\
\hline 33 & $\mathrm{H}$ & 10.643729 & -1.648365 & -0.298778 & 90 & $\mathrm{C}$ & -5.896365 & 2.457688 & 4.636540 \\
\hline 34 & C & 10.609636 & 1.564553 & 1.021631 & 91 & $\mathrm{H}$ & -5.995838 & 1.407909 & 4.932467 \\
\hline 35 & $\mathrm{H}$ & 11.697292 & 1.692051 & 1.052679 & 92 & $\mathrm{H}$ & -4.898565 & 2.796848 & 4.931545 \\
\hline 36 & $\mathrm{H}$ & 10.161797 & 2.525968 & 0.749453 & 93 & $\mathrm{H}$ & -6.629142 & 3.042627 & 5.204455 \\
\hline 37 & $\mathrm{H}$ & 10.274720 & 1.309450 & 2.031581 & 94 & $\mathrm{C}$ & 3.767616 & -1.308831 & 0.534648 \\
\hline 38 & C & -1.818464 & 4.620706 & -2.171711 & 95 & C & 2.375591 & -1.330535 & 0.458801 \\
\hline 39 & C & -1.276008 & 5.903389 & -2.807700 & 96 & $\mathrm{Au}$ & -0.287723 & -0.366899 & 0.082184 \\
\hline 40 & $\mathrm{H}$ & -2.111205 & 6.557425 & -3.077942 & 97 & C & 0.138535 & -2.231039 & 0.909619 \\
\hline 41 & $\mathrm{H}$ & -0.630901 & 6.458949 & -2.118908 & 98 & $\mathrm{C}$ & 1.540906 & -2.430001 & 0.919493 \\
\hline 42 & $\mathrm{H}$ & -0.708123 & 5.696300 & -3.720994 & 99 & $\mathrm{C}$ & 2.145098 & -3.631963 & 1.300574 \\
\hline 43 & C & -2.623184 & 5.004844 & -0.917898 & 100 & C & -0.064740 & -4.468457 & 1.811788 \\
\hline 44 & $\mathrm{H}$ & -3.062500 & 4.129301 & -0.430110 & 101 & C & 1.316453 & -4.655141 & 1.761336 \\
\hline 45 & $\mathrm{H}$ & -1.986043 & 5.513636 & -0.186742 & 102 & $\mathrm{H}$ & -0.696225 & -5.273459 & 2.179478 \\
\hline 46 & $\mathrm{H}$ & -3.439270 & 5.684015 & -1.188652 & 103 & $\mathrm{H}$ & 1.748926 & -5.603060 & 2.071559 \\
\hline 47 & C & -2.749712 & 3.935832 & -3.186096 & 104 & $\mathrm{C}$ & 3.629505 & -3.788564 & 1.077702 \\
\hline 48 & $\mathrm{H}$ & -2.201862 & 3.652553 & -4.090970 & 105 & $\mathrm{H}$ & 4.042122 & -4.539139 & 1.758707 \\
\hline 49 & $\mathrm{H}$ & -3.210465 & 3.033738 & -2.772858 & 106 & $\mathrm{H}$ & 3.774870 & -4.182749 & 0.061631 \\
\hline 50 & $\mathrm{H}$ & -3.555408 & 4.619317 & -3.475995 & 107 & C & 4.419487 & -2.482760 & 1.224012 \\
\hline 51 & C & -3.115696 & -1.269635 & -0.497731 & 108 & $\mathrm{H}$ & 4.509431 & -2.241393 & 2.292855 \\
\hline 52 & C & -3.077257 & 0.394990 & 0.973225 & 109 & $\mathrm{H}$ & 5.438968 & -2.624161 & 0.855074 \\
\hline 53 & $\mathrm{C}$ & -4.474025 & -1.002528 & -0.171397 & 110 & $\mathrm{C}$ & -2.803390 & -2.268984 & -1.422519 \\
\hline 54 & C & -2.712464 & 1.415256 & 1.859875 & 111 & $\mathrm{H}$ & -1.770646 & -2.481253 & -1.686264 \\
\hline 55 & C & -4.447279 & 0.078419 & 0.781718 & 112 & $\mathrm{C}$ & -0.654365 & -3.274285 & 1.376479 \\
\hline 56 & C & -3.843610 & -2.991022 & -1.996739 & 113 & $\mathrm{H}$ & -1.736551 & -3.174054 & 1.391920 \\
\hline 57 & C & -5.49452 & 1. & & & & & & \\
\hline
\end{tabular}


Table S13 Cartesian coordinates of the optimized $\mathrm{T}_{1}$ geometry of $\mathbf{1}$

\begin{tabular}{|c|c|c|c|c|c|c|c|c|c|}
\hline 1 & C & -0.014802 & 1.952854 & 0.515214 & 57 & $\mathrm{C}$ & 3.336936 & -3.883942 & 0.510238 \\
\hline 2 & $\mathrm{C}$ & -1.414780 & 2.236020 & 0.548137 & 58 & $\mathrm{C}$ & 5.227040 & -2.429809 & 0.133601 \\
\hline 3 & C & 0.857215 & 2.980096 & 0.872853 & 59 & $\mathrm{C}$ & 4.222998 & 2.526261 & -1.546450 \\
\hline 4 & $\mathrm{C}$ & -1.856459 & 3.527665 & 0.841814 & 60 & $\mathrm{H}$ & 2.097941 & 2.231847 & -1.291376 \\
\hline 5 & $\mathrm{C}$ & 0.426443 & 4.280671 & 1.192071 & 61 & $\mathrm{C}$ & 5.682180 & 0.707022 & -0.915579 \\
\hline 6 & $\mathrm{H}$ & 1.923466 & 2.771017 & 0.913068 & 62 & C & 4.713067 & -3.708506 & 0.486686 \\
\hline 7 & $\mathrm{C}$ & -0.948632 & 4.536219 & 1.145748 & 63 & $\mathrm{H}$ & 2.950939 & -4.864779 & 0.751413 \\
\hline 8 & $\mathrm{H}$ & -2.921213 & 3.748551 & 0.861514 & 64 & $\mathrm{H}$ & 6.298671 & -2.271106 & 0.058300 \\
\hline 9 & $\mathrm{H}$ & -1.330800 & 5.525282 & 1.374507 & 65 & C & 5.528018 & 2.009685 & -1.416113 \\
\hline 0 & $\mathrm{C}$ & -2.307007 & 1.098193 & 0.346873 & 66 & $\mathrm{H}$ & 4.084303 & 3.517061 & -1.968385 \\
\hline 11 & $\mathrm{C}$ & -3.672715 & 1.048708 & 0.204289 & 67 & $\mathrm{H}$ & 6.667406 & 0.261701 & -0.830943 \\
\hline 12 & C & -4.373058 & -0.179364 & -0.027487 & 68 & $\mathrm{~N}$ & 2.317643 & -0.377789 & -0.220785 \\
\hline 13 & $\mathrm{H}$ & -4.235714 & 1.976455 & 0.212928 & 69 & $\mathrm{C}$ & 5.693243 & -4.835915 & 0.798959 \\
\hline 14 & $\mathrm{~N}$ & -1.612284 & -0.087344 & 0.325589 & 70 & $\mathrm{C}$ & 4.987469 & -6.144033 & 1.163459 \\
\hline 15 & $\mathrm{C}$ & -5.834027 & -0.128059 & -0.189050 & 71 & $\mathrm{H}$ & 4.355553 & -6.032950 & 2.050830 \\
\hline 16 & C & -6.609705 & 0.720491 & 0.617687 & 72 & $\mathrm{H}$ & 4.368995 & -6.517624 & 0.340620 \\
\hline 17 & $\mathrm{C}$ & -6.522324 & -0.875760 & -1.162077 & 73 & $\mathrm{H}$ & 5.737420 & -6.909371 & 1.385540 \\
\hline 18 & $\mathrm{C}$ & -7.990599 & 0.807034 & 0.472075 & 74 & C & 6.576948 & -4.413930 & 1.986378 \\
\hline 19 & $\mathrm{H}$ & -6.123536 & 1.307351 & 1.392880 & 75 & $\mathrm{H}$ & 7.294755 & -5.208977 & 2.214749 \\
\hline 20 & $\mathrm{C}$ & -7.900714 & -0.785743 & -1.300495 & 76 & $\mathrm{H}$ & 7.145807 & -3.504004 & 1.772853 \\
\hline 21 & $\mathrm{H}$ & -5.967205 & -1.509976 & -1.846006 & 77 & $\mathrm{H}$ & 5.971404 & -4.233706 & 2.880399 \\
\hline 22 & $\mathrm{C}$ & -8.676002 & 0.054636 & -0.487963 & 78 & C & 6.579095 & -5.089053 & -0.433843 \\
\hline 23 & $\mathrm{H}$ & -8.535551 & 1.472993 & 1.133311 & 79 & $\mathrm{H}$ & 5.974717 & -5.395436 & -1.293629 \\
\hline 24 & $\mathrm{H}$ & -8.379913 & -1.378907 & -2.075547 & 80 & $\mathrm{H}$ & 7.151370 & -4.201563 & -0.720167 \\
\hline 25 & $\mathrm{C}$ & -10.192056 & 0.123185 & -0.679213 & 81 & $\mathrm{H}$ & 7.294706 & -5.889287 & -0.216916 \\
\hline 26 & $\mathrm{C}$ & -10.857805 & 1.083178 & 0.310368 & 82 & $\mathrm{C}$ & 6.719878 & 2.869034 & -1.838691 \\
\hline 27 & $\mathrm{H}$ & -10.691449 & 0.776760 & 1.348579 & 83 & $\mathrm{C}$ & 8.056690 & 2.153013 & -1.628244 \\
\hline 28 & $\mathrm{H}$ & -10.491616 & 2.108473 & 0.191866 & 84 & $\mathrm{H}$ & 8.219124 & 1.893670 & -0.576744 \\
\hline 29 & $\mathrm{H}$ & -11.938836 & 1.095579 & 0.137313 & 85 & $\mathrm{H}$ & 8.128747 & 1.238287 & -2.226069 \\
\hline 30 & $\mathrm{C}$ & -10.797535 & -1.275517 & -0.475173 & 86 & $\mathrm{H}$ & 8.873875 & 2.812814 & -1.935831 \\
\hline 31 & $\mathrm{H}$ & -11.883420 & -1.242920 & -0.619407 & 87 & C & 6.729016 & 4.162984 & -1.006334 \\
\hline 32 & $\mathrm{H}$ & -10.388419 & -2.002163 & -1.183766 & 88 & $\mathrm{H}$ & 5.813244 & 4.745256 & -1.145908 \\
\hline 33 & $\mathrm{H}$ & -10.599474 & -1.643201 & 0.537173 & 89 & $\mathrm{H}$ & 6.829260 & 3.940837 & 0.061119 \\
\hline 34 & C & -10.504635 & 0.607250 & -2.105117 & 90 & $\mathrm{H}$ & 7.574355 & 4.792779 & -1.304289 \\
\hline 35 & $\mathrm{H}$ & -11.588481 & 0.653082 & -2.260814 & 91 & C & 6.590349 & 3.221948 & -3.330510 \\
\hline 36 & $\mathrm{H}$ & -10.093309 & 1.607505 & -2.276940 & 92 & $\mathrm{H}$ & 6.583621 & 2.317508 & -3.947475 \\
\hline 37 & $\mathrm{H}$ & -10.086540 & -0.063599 & -2.861872 & 93 & $\mathrm{H}$ & 5.674470 & 3.782100 & -3.540674 \\
\hline 38 & C & 1.452506 & 5.348516 & 1.581386 & 94 & $\mathrm{H}$ & 7.438700 & 3.840947 & -3.642126 \\
\hline 39 & $\mathrm{C}$ & 0.797699 & 6.698452 & 1.888175 & 95 & $\mathrm{C}$ & -3.613468 & -1.376069 & -0.087117 \\
\hline 40 & $\mathrm{H}$ & 1.569757 & 7.425678 & 2.160046 & 96 & C & -2.242291 & -1.293283 & 0.092635 \\
\hline 41 & $\mathrm{H}$ & 0.258775 & 7.095638 & 1.021421 & 97 & $\mathrm{Au}$ & 0.334680 & -0.087701 & 0.131760 \\
\hline 42 & $\mathrm{H}$ & 0.096814 & 6.630140 & 2.726864 & 98 & $\mathrm{C}$ & 0.039906 & -2.110326 & 0.026325 \\
\hline 43 & $\mathrm{C}$ & 2.449827 & 5.551031 & 0.428535 & 99 & C & -1.341983 & -2.405165 & 0.043608 \\
\hline 44 & $\mathrm{H}$ & 2.993620 & 4.630375 & 0.196445 & 100 & C & 0.969587 & -3.156671 & 0.125216 \\
\hline 45 & $\mathrm{H}$ & 1.934790 & 5.878596 & -0.480934 & 101 & $\mathrm{C}$ & -1.826029 & -3.722568 & 0.096388 \\
\hline 46 & $\mathrm{H}$ & 3.187439 & 6.316378 & 0.696151 & 102 & $\mathrm{C}$ & 0.477084 & -4.486233 & 0.114765 \\
\hline 47 & $\mathrm{C}$ & 2.215016 & 4.888987 & 2.835478 & 103 & C & -0.892057 & -4.755772 & 0.097348 \\
\hline 48 & $\mathrm{H}$ & 1.530453 & 4.747903 & 3.678557 & 104 & $\mathrm{H}$ & 1.162565 & -5.326786 & 0.117264 \\
\hline 49 & $\mathrm{H}$ & 2.741777 & 3.944738 & 2.666803 & 105 & $\mathrm{H}$ & -1.229435 & -5.789261 & 0.115671 \\
\hline 50 & $\mathrm{H}$ & 2.959193 & 5.640459 & 3.123079 & 106 & C & -3.311346 & -3.885263 & 0.274451 \\
\hline 51 & $\mathrm{C}$ & 2.946254 & -1.566809 & 0.010785 & 107 & $\mathrm{H}$ & -3.645801 & -4.858453 & -0.099611 \\
\hline 52 & $\mathrm{C}$ & 3.272532 & 0.539411 & -0.633277 & 108 & $\mathrm{H}$ & -3.512891 & -3.884696 & 1.356762 \\
\hline 53 & C & 2.396490 & -2.862040 & 0.236151 & 109 & C & -4.141644 & -2.766117 & -0.375295 \\
\hline 54 & $\mathrm{C}$ & 4.362214 & -1.395520 & -0.111177 & 110 & $\mathrm{H}$ & -4.154695 & -2.919679 & -1.464386 \\
\hline 55 & C & 3.091405 & 1.817108 & -1.164051 & 111 & $\mathrm{H}$ & -5.180060 & -2.855344 & -0.043561 \\
\hline 56 & $\mathrm{C}$ & 4.563412 & -0.024800 & -0.545655 & & & & & \\
\hline
\end{tabular}


Table S14 Cartesian coordinates of the optimized $\mathrm{T}_{1}$ geometry of 2.

\begin{tabular}{|c|c|c|c|c|c|c|c|c|c|}
\hline 1 & $\mathrm{C}$ & -0.560941 & 2.443551 & -0.149702 & 57 & C & 6.322986 & -2.143947 & -0.204202 \\
\hline 2 & C & -1.993330 & 2.400642 & -0.162985 & 58 & C & 2.947598 & 1.880330 & 0.579920 \\
\hline 3 & $\mathrm{C}$ & 0.032057 & 3.685444 & -0.374107 & 59 & $\mathrm{C}$ & 5.127676 & 0.856158 & 0.483242 \\
\hline 4 & C & -2.727933 & 3.570666 & -0.351336 & 60 & C & 6.544300 & -3.481619 & -0.581662 \\
\hline 5 & C & -0.695003 & 4.873293 & -0.576669 & 61 & $\mathrm{H}$ & 5.559839 & -5.317489 & -1.136222 \\
\hline 6 & $\mathrm{H}$ & 1.116376 & 3.745683 & -0.412631 & 62 & $\mathrm{H}$ & 7.159798 & -1.462669 & -0.081498 \\
\hline 7 & C & -2.089819 & 4.790767 & -0.549154 & 63 & C & 4.346085 & 2.002886 & 0.677465 \\
\hline 8 & $\mathrm{H}$ & -3.814920 & 3.536035 & -0.354151 & 64 & $\mathrm{H}$ & 2.348955 & 2.774593 & 0.708489 \\
\hline 9 & $\mathrm{H}$ & -2.697206 & 5.677532 & -0.694208 & 65 & $\mathrm{H}$ & 6.210073 & 0.901216 & 0.519930 \\
\hline 10 & $\mathrm{C}$ & -2.622476 & 1.081507 & -0.013993 & 66 & $\mathrm{~N}$ & 2.750647 & -1.819053 & 0.097874 \\
\hline 11 & $\mathrm{C}$ & -3.951922 & 0.720890 & -0.029362 & 67 & $\mathrm{C}$ & 7.975816 & -3.979311 & -0.778543 \\
\hline 12 & $\mathrm{C}$ & -4.349261 & -0.656074 & 0.048918 & 68 & $\mathrm{C}$ & 8.028792 & -5.452217 & -1.192168 \\
\hline 13 & $\mathrm{H}$ & -4.717609 & 1.478639 & -0.155816 & 69 & $\mathrm{H}$ & 7.572072 & -6.103615 & -0.439725 \\
\hline 14 & $\mathrm{~N}$ & -1.692244 & 0.104821 & 0.129177 & 70 & $\mathrm{H}$ & 7.528486 & -5.625648 & -2.150649 \\
\hline 15 & C & -5.791111 & -0.964661 & -0.007928 & 71 & $\mathrm{H}$ & 9.073154 & -5.759005 & -1.305509 \\
\hline 16 & C & -6.711074 & -0.185240 & 0.707983 & 72 & C & 8.746976 & -3.818853 & 0.543056 \\
\hline 17 & $\mathrm{C}$ & -6.313429 & -2.008183 & -0.789951 & 73 & $\mathrm{H}$ & 9.778870 & -4.163778 & 0.416516 \\
\hline 18 & $\mathrm{C}$ & -8.077590 & -0.444053 & 0.660066 & 74 & $\mathrm{H}$ & 8.781860 & -2.775851 & 0.871890 \\
\hline 19 & $\mathrm{H}$ & -6.347566 & 0.626649 & 1.332770 & 75 & $\mathrm{H}$ & 8.285238 & -4.410228 & 1.340382 \\
\hline 20 & C & -7.678306 & -2.260596 & -0.834221 & 76 & C & 8.659302 & -3.143217 & -1.874124 \\
\hline 21 & $\mathrm{H}$ & -5.646365 & -2.610457 & -1.398978 & 77 & $\mathrm{H}$ & 8.130075 & -3.239592 & -2.827651 \\
\hline 22 & C & -8.598594 & -1.489644 & -0.109581 & 78 & $\mathrm{H}$ & 8.699240 & -2.081108 & -1.614587 \\
\hline 23 & $\mathrm{H}$ & -8.740560 & 0.185551 & 1.244406 & 79 & $\mathrm{H}$ & 9.688373 & -3.489391 & -2.018700 \\
\hline 24 & $\mathrm{H}$ & -8.031985 & -3.074622 & -1.462002 & 80 & C & 4.969148 & 3.371224 & 0.958519 \\
\hline 25 & C & -10.093462 & -1.804889 & -0.188134 & 81 & C & 6.490215 & 3.293128 & 1.120680 \\
\hline 26 & $\mathrm{C}$ & -10.931505 & -0.857565 & 0.674586 & 82 & $\mathrm{H}$ & 6.777776 & 2.630839 & 1.944127 \\
\hline 27 & $\mathrm{H}$ & -10.659308 & -0.923402 & 1.733340 & 83 & $\mathrm{H}$ & 6.982149 & 2.943832 & 0.206886 \\
\hline 28 & $\mathrm{H}$ & -10.824633 & 0.184833 & 0.356232 & 84 & $\mathrm{H}$ & 6.882743 & 4.290157 & 1.343597 \\
\hline 29 & $\mathrm{H}$ & -11.989925 & -1.123576 & 0.587372 & 85 & C & 4.378676 & 3.950119 & 2.255176 \\
\hline 30 & C & -10.337564 & -3.243202 & 0.298109 & 86 & $\mathrm{H}$ & 3.294698 & 4.083380 & 2.191697 \\
\hline 31 & $\mathrm{H}$ & -11.404400 & -3.486598 & 0.238876 & 87 & $\mathrm{H}$ & 4.589167 & 3.295475 & 3.107247 \\
\hline 32 & $\mathrm{H}$ & -9.794170 & -3.974411 & -0.308163 & 88 & $\mathrm{H}$ & 4.822391 & 4.930213 & 2.460972 \\
\hline 33 & $\mathrm{H}$ & -10.018213 & -3.363493 & 1.338673 & 89 & $\mathrm{C}$ & 4.658733 & 4.316243 & -0.214825 \\
\hline 34 & C & -10.566376 & -1.675309 & -1.645700 & 90 & $\mathrm{H}$ & 5.078311 & 3.930875 & -1.149832 \\
\hline 35 & $\mathrm{H}$ & -11.634911 & -1.907258 & -1.718584 & 91 & $\mathrm{H}$ & 3.581861 & 4.445651 & -0.357638 \\
\hline 36 & $\mathrm{H}$ & -10.412898 & -0.656706 & -2.017242 & 92 & $\mathrm{H}$ & 5.092693 & 5.304415 & -0.026829 \\
\hline 37 & $\mathrm{H}$ & -10.030002 & -2.360631 & -2.309064 & 93 & C & -3.355463 & -1.640169 & 0.189307 \\
\hline 38 & C & 0.046008 & 6.191992 & -0.815252 & 94 & C & -2.023136 & -1.218948 & 0.255585 \\
\hline 39 & C & -0.912838 & 7.362057 & -1.055395 & 95 & $\mathrm{Au}$ & 0.260659 & 0.527290 & 0.132989 \\
\hline 40 & $\mathrm{H}$ & -0.336486 & 8.276875 & -1.227818 & 96 & C & 0.386333 & -1.495931 & 0.294758 \\
\hline 41 & $\mathrm{H}$ & -1.544223 & 7.196410 & -1.934725 & 97 & C & -0.900214 & -2.079253 & 0.402390 \\
\hline 42 & $\mathrm{H}$ & -1.563923 & 7.539245 & -0.192902 & 98 & $\mathrm{C}$ & 1.496953 & -2.352625 & 0.330527 \\
\hline 43 & C & 0.951890 & 6.058683 & -2.050673 & 99 & C & -1.079106 & -3.456111 & 0.705690 \\
\hline 44 & $\mathrm{H}$ & 1.694223 & 5.263638 & -1.931693 & 100 & C & 1.330815 & -3.738168 & 0.638038 \\
\hline 45 & $\mathrm{H}$ & 0.360782 & 5.834542 & -2.944923 & 101 & C & 0.051953 & -4.258932 & 0.827920 \\
\hline 46 & $\mathrm{H}$ & 1.492454 & 6.995697 & -2.225878 & 102 & $\mathrm{H}$ & 2.190054 & -4.369130 & 0.816996 \\
\hline 47 & C & 0.904816 & 6.525308 & 0.416143 & 103 & $\mathrm{H}$ & -0.054901 & -5.303175 & 1.108860 \\
\hline 48 & $\mathrm{H}$ & 0.279664 & 6.640016 & 1.307963 & 104 & C & -2.479959 & -3.882732 & 1.033788 \\
\hline 49 & $\mathrm{H}$ & 1.642155 & 5.743691 & 0.622391 & 105 & $\mathrm{H}$ & -2.598965 & -4.963732 & 0.908455 \\
\hline 50 & $\mathrm{H}$ & 1.448154 & 7.463787 & 0.257640 & 106 & $\mathrm{H}$ & -2.637486 & -3.676367 & 2.104209 \\
\hline 51 & C & 3.932680 & -2.545331 & -0.154516 & 107 & C & -3.563566 & -3.137538 & 0.234390 \\
\hline 52 & C & 3.093590 & -0.450060 & 0.228561 & 108 & $\mathrm{H}$ & -3.566062 & -3.522673 & -0.795444 \\
\hline 53 & C & 5.034584 & -1.680214 & -0.011730 & 109 & $\mathrm{H}$ & -4.545491 & -3.378428 & 0.651574 \\
\hline 54 & C & 2.265157 & 0.682903 & 0.328182 & 110 & C & 4.118734 & -3.850355 & -0.600905 \\
\hline 55 & C & 4.499665 & -0.355859 & 0.253035 & 111 & $\mathrm{H}$ & 3.289884 & -4.503402 & -0.841622 \\
\hline 56 & C & 5.425734 & -4.298537 & -0.792933 & & & & & \\
\hline
\end{tabular}




\section{Table S15 Cartesian coordinates of the optimized $\mathrm{T}_{1}$ geometry of 5 .}

\begin{tabular}{|c|c|c|c|c|c|c|c|c|}
\hline 1 & -0.051279 & 1.472083 & -0.858278 & 58 & C & -3.560490 & 1.905991 & 2.776972 \\
\hline 2 & 1.320748 & 1.755967 & -1.117854 & 59 & $\mathrm{H}$ & -1.547303 & 1.453319 & 2.130146 \\
\hline 3 & -1.013525 & 2.412536 & -1.212332 & 60 & C & -5.373178 & 0.737317 & 1.665200 \\
\hline 4 & 1.658730 & 2.972427 & -1.720891 & 61 & C & -5.300889 & -2.502843 & -1.836052 \\
\hline 5 & -0.682217 & 3.643435 & -1.803351 & 62 & $\mathrm{H}$ & -3.743650 & -3.450167 & -2.986710 \\
\hline 6 & -2.060595 & 2.191298 & -1.015340 & 63 & $\mathrm{H}$ & -6.592630 & -1.370768 & -0.502785 \\
\hline 7 & 0.674304 & 3.896663 & -2.051689 & 64 & C & -4.943776 & 1.671448 & 2.629815 \\
\hline 8 & 2.698752 & 3.204446 & -1.939100 & 65 & $\mathrm{H}$ & -3.219790 & 2.623386 & 3.516691 \\
\hline 9 & 0.978384 & 4.829289 & -2.515100 & 66 & $\mathrm{H}$ & -6.429784 & 0.536523 & 1.528414 \\
\hline 10 & 2.270622 & 0.734896 & -0.701795 & 67 & $\mathrm{~N}$ & -2.313649 & -0.420941 & 0.180401 \\
\hline 11 & 3.645126 & 0.790763 & -0.628186 & 68 & C & -6.472352 & -3.210983 & -2.513235 \\
\hline 12 & 4.422113 & -0.258927 & -0.065330 & 69 & C & -6.016936 & -4.179972 & -3.607237 \\
\hline 13 & 4.148627 & 1.694561 & -0.957200 & 70 & $\mathrm{H}$ & -5.473197 & -3.666398 & -4.406919 \\
\hline 14 & 1.645127 & -0.425424 & -0.287473 & 71 & $\mathrm{H}$ & -5.378505 & -4.975092 & -3.208391 \\
\hline 15 & 5.884254 & -0.094220 & -0.018175 & 72 & $\mathrm{H}$ & -6.894228 & -4.655186 & -4.056738 \\
\hline 16 & 6.569496 & 0.460521 & -1.111012 & 73 & C & -7.395353 & -2.158040 & -3.152083 \\
\hline 17 & 6.658945 & -0.431702 & 1.106382 & 74 & $\mathrm{H}$ & -8.244535 & -2.653669 & -3.634627 \\
\hline 18 & 7.946633 & 0.657932 & -1.087646 & 75 & $\mathrm{H}$ & -7.795274 & -1.458712 & -2.411819 \\
\hline 19 & 6.013800 & 0.722633 & -2.007859 & 76 & $\mathrm{H}$ & -6.861382 & -1.578958 & -3.912341 \\
\hline 20 & 8.033215 & -0.234798 & 1.121846 & 77 & $\mathrm{C}$ & -7.258949 & -4.007395 & -1.457103 \\
\hline 21 & 6.175670 & -0.821176 & 1.996708 & 78 & $\mathrm{H}$ & -6.625584 & -4.766828 & -0.987477 \\
\hline 22 & 8.718149 & 0.312994 & 0.027203 & 79 & $\mathrm{H}$ & -7.657115 & -3.362306 & -0.668246 \\
\hline 23 & 8.419347 & 1.082430 & -1.967455 & 80 & $\mathrm{H}$ & -8.105879 & -4.514985 & -1.931121 \\
\hline 24 & 8.581615 & -0.504948 & 2.021016 & 81 & $\mathrm{C}$ & -5.928667 & 2.436675 & 3.512124 \\
\hline 25 & 10.233464 & 0.512144 & 0.090461 & 82 & C & -7.384515 & 2.057980 & 3.226818 \\
\hline 26 & 10.791924 & 1.120521 & -1.198791 & 83 & $\mathrm{H}$ & -7.671637 & 2.285390 & 2.194819 \\
\hline 27 & 10.604299 & 0.478694 & -2.066127 & 84 & $\mathrm{H}$ & -7.574861 & 0.996049 & 3.414705 \\
\hline 28 & 10.362537 & 2.107256 & -1.402253 & 85 & $\mathrm{H}$ & -8.042747 & 2.632360 & 3.885776 \\
\hline 29 & 11.875890 & 1.243581 & -1.105895 & 86 & C & -5.762452 & 3.945813 & 3.259863 \\
\hline 30 & 10.917967 & -0.845742 & 0.318859 & 87 & $\mathrm{H}$ & -4.751551 & 4.291358 & 3.494874 \\
\hline 31 & 12.004867 & -0.717627 & 0.376164 & 88 & $\mathrm{H}$ & -5.971130 & 4.193597 & 2.214046 \\
\hline 32 & 10.587237 & -1.314782 & 1.250582 & 89 & $\mathrm{H}$ & -6.462093 & 4.506087 & 3.889371 \\
\hline 33 & 10.699375 & -1.536362 & -0.502433 & 90 & $\mathrm{C}$ & -5.629515 & 2.128520 & 4.989837 \\
\hline 34 & 10.576609 & 1.455038 & 1.255896 & 91 & $\mathrm{H}$ & -5.743344 & 1.059767 & 5.197975 \\
\hline 35 & 11.661048 & 1.599941 & 1.319393 & 92 & $\mathrm{H}$ & -4.614918 & 2.423243 & 5.273147 \\
\hline 36 & 10.109741 & 2.435604 & 1.115342 & 93 & $\mathrm{H}$ & -6.327752 & 2.677059 & 5.631055 \\
\hline 37 & 10.236649 & 1.054362 & 2.215761 & 94 & C & 3.732028 & -1.399593 & 0.436726 \\
\hline 38 & -1.790085 & 4.644106 & -2.142955 & 95 & C & 2.355476 & -1.442445 & 0.312099 \\
\hline 39 & -1.242984 & 5.918565 & -2.791961 & 96 & $\mathrm{Au}$ & -0.298152 & -0.402186 & 0.005245 \\
\hline 40 & -2.070560 & 6.599623 & -3.016041 & 97 & $\mathrm{C}$ & 0.121478 & -2.244839 & 0.888224 \\
\hline 41 & -0.549051 & 6.446927 & -2.129689 & 98 & C & 1.517967 & -2.488919 & 0.839637 \\
\hline 42 & -0.724726 & 5.705326 & -3.732886 & 99 & $\mathrm{C}$ & 2.089678 & -3.695028 & 1.272122 \\
\hline 43 & -2.530033 & 5.041032 & -0.854019 & 100 & C & -0.127858 & -4.435579 & 1.897750 \\
\hline 44 & -2.976998 & 4.173506 & -0.358407 & 101 & C & 1.252039 & -4.662921 & 1.814136 \\
\hline 45 & -1.846571 & 5.519085 & -0.144183 & 102 & $\mathrm{H}$ & -0.769989 & -5.198445 & 2.331291 \\
\hline 46 & -3.335001 & 5.749461 & -1.081060 & 103 & $\mathrm{H}$ & 1.668495 & -5.605526 & 2.162647 \\
\hline 47 & -2.785514 & 3.997439 & -3.120657 & 104 & C & 3.564389 & -3.873992 & 1.015238 \\
\hline & -2.286820 & 3.710996 & -4.052651 & 105 & $\mathrm{H}$ & 3.984345 & -4.637290 & 1.678693 \\
\hline & -3.247923 & 3.101023 & -2.696252 & 106 & $\mathrm{H}$ & 3.686418 & -4.252547 & -0.010806 \\
\hline 50 & -3.587734 & 4.702534 & -3.366649 & 107 & C & 4.364981 & -2.572789 & 1.153534 \\
\hline 51 & -3.188713 & -1.177929 & -0.568040 & 108 & $\mathrm{H}$ & 4.459538 & -2.325106 & 2.221643 \\
\hline 52 & -3.057624 & 0.333518 & 1.056649 & 109 & $\mathrm{H}$ & 5.383693 & -2.735601 & 0.788958 \\
\hline 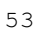 & -4.528798 & -0.917582 & -0.177909 & 110 & C & -2.899672 & -2.091847 & -1.579598 \\
\hline & -2.607635 & 1.255078 & 2.007413 & 111 & $\mathrm{H}$ & -1.873521 & -2.292907 & -1.872455 \\
\hline & -4.441612 & 0.075877 & 0.887168 & 112 & $\mathrm{C}$ & -0.690628 & -3.246134 & 1.424637 \\
\hline & -3.966864 & -2.740131 & -2.199434 & 113 & $\mathrm{H}$ & -1.768633 & -3.109543 & 1.483015 \\
\hline & -5.568159 & -1.571015 & -0.802674 & & & & & \\
\hline
\end{tabular}




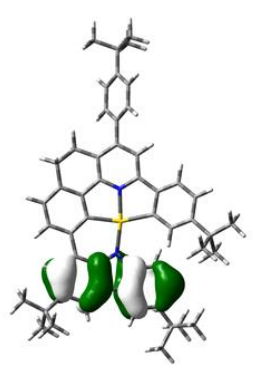

HOMO-1

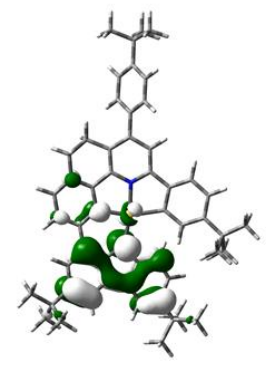

HOMO

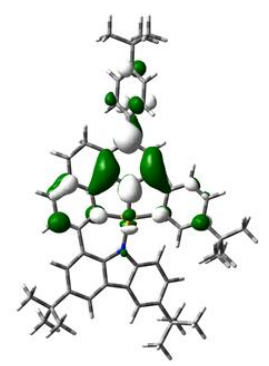

LUMO

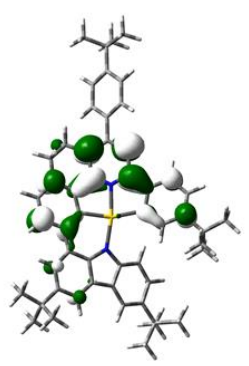

LUMO+1

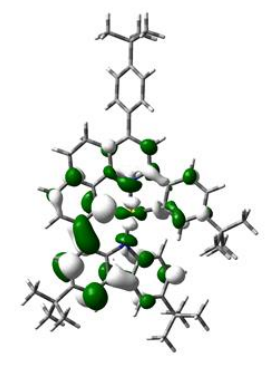

LUMO+2

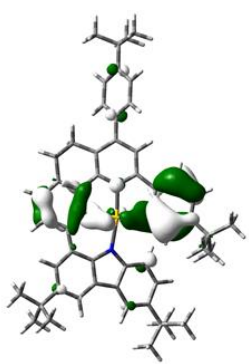

HOMO-6

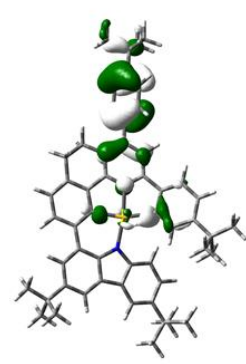

HOMO-5

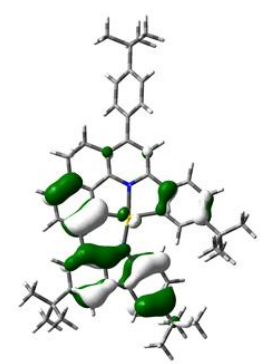

HOMO-3

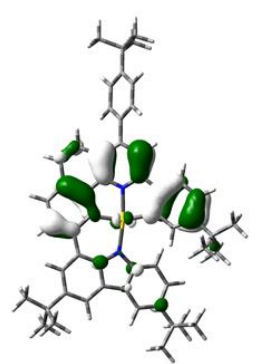

HOMO-2

Figure S16 Spatial plots (isovalue $=0.03$ ) of selected molecular orbitals of $\mathbf{1}$ at the optimized ground-state geometry.

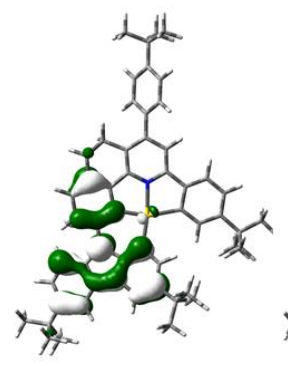

HOMO

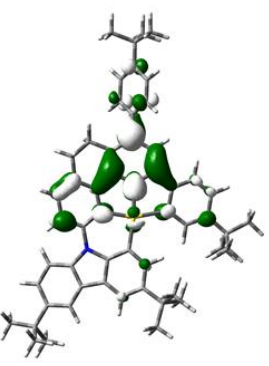

LUMO

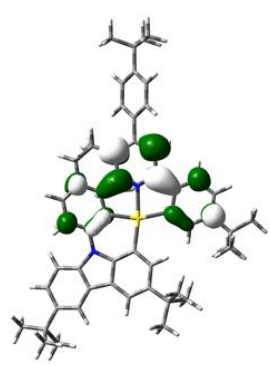

LUMO+1

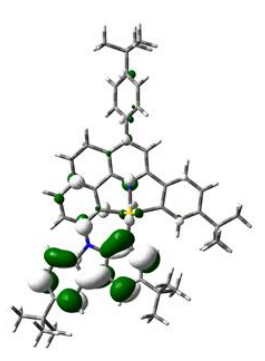

LUMO+2

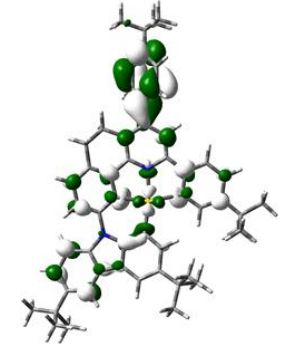

LUMO+3

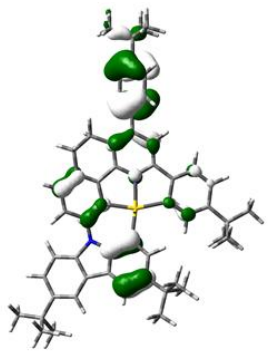

HOMO-5

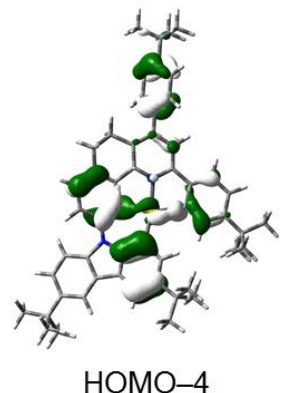

HOMO-4

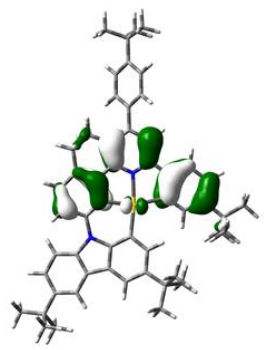

HOMO-2

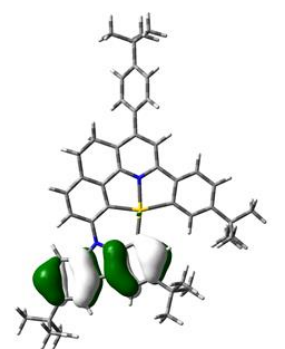

HOMO-1

Figure S17 Spatial plots (isovalue $=0.03$ ) of selected molecular orbitals of 2 at the optimized ground-state geometry. 


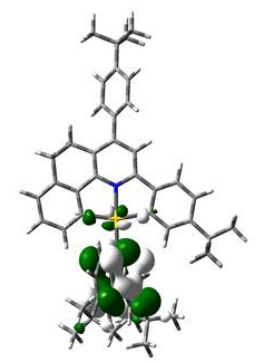

HOMO

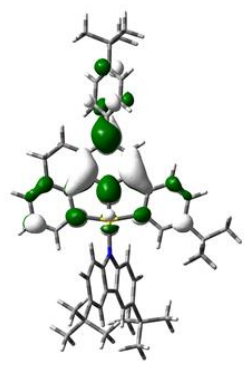

LUMO

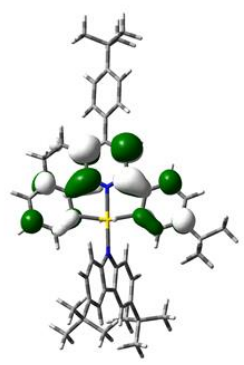

LUMO+1

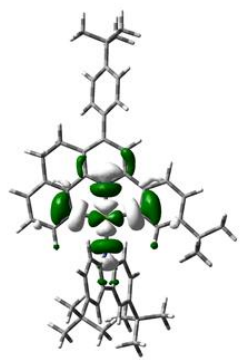

LUMO+2

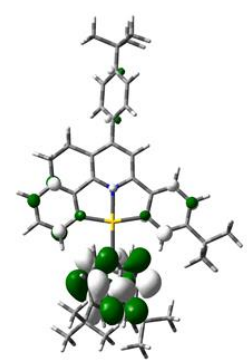

$\mathrm{LUMO}+3$

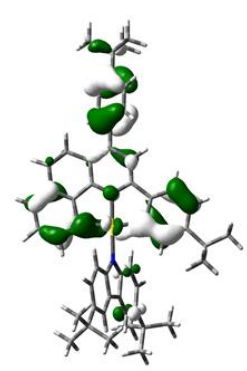

HOMO-6

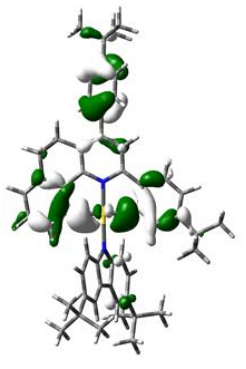

HOMO-5

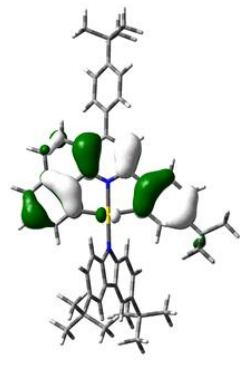

HOMO-2

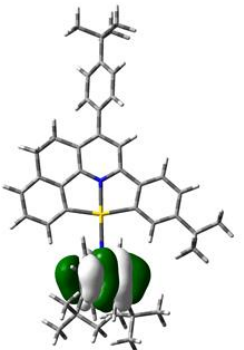

HOMO-1

Figure S18 Spatial plots (isovalue $=0.03$ ) of selected molecular orbitals of 5 at the optimized ground-state geometry. 


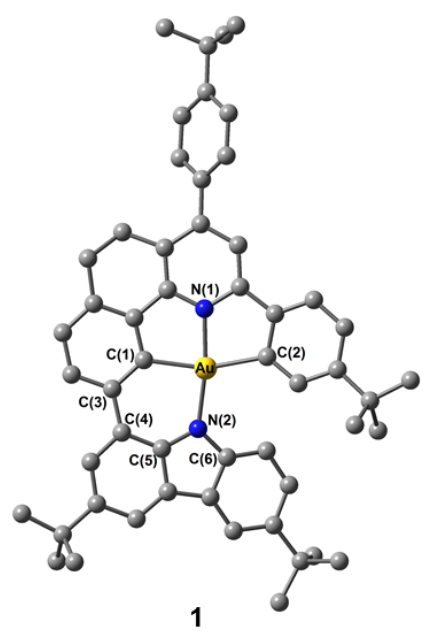

$\mathrm{Au}-\mathrm{C}(1) \quad 2.047 \mathrm{C}(1)-\mathrm{Au}-\mathrm{N}(1) \quad 82.1$

$\mathrm{Au}-\mathrm{C}(2) 2.105 \mathrm{C}(2)-\mathrm{Au}-\mathrm{N}(1) 79.4$

$\mathrm{Au}-\mathrm{N}(1) 1.957 \mathrm{~N}(1)-\mathrm{Au}-\mathrm{N}(2) \quad 170.7$

$\mathrm{Au}-\mathrm{N}(2) 2.035 \mathrm{C}(1)-\mathrm{C}(3)-\mathrm{C}(4)-\mathrm{C}(5) 11.7$

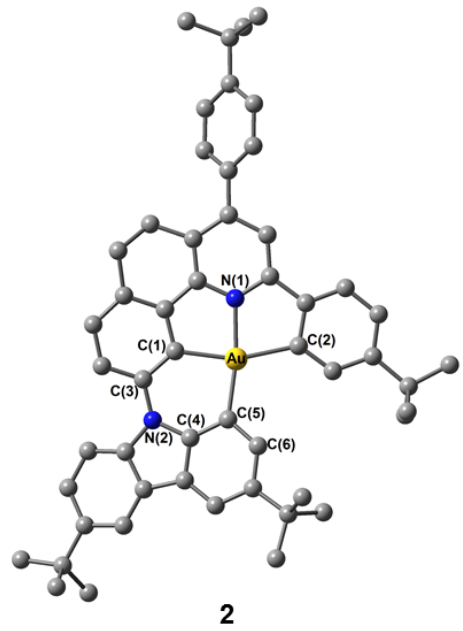

$A u-C(1) 2.034 C(1)-A u-N(1) \quad 81.4$ $\mathrm{Au}-\mathrm{C}(2) 2.104 \mathrm{C}(2)-\mathrm{Au}-\mathrm{N}(1) 79.1$

$\mathrm{Au}-\mathrm{N}(1) 1.998 \mathrm{~N}(1)-\mathrm{Au}-\mathrm{C}(5) 170.5$

$\mathrm{Au}-\mathrm{C}(5) \quad 2.020 \mathrm{C}(1)-\mathrm{C}(3)-\mathrm{N}(2)-\mathrm{C}(4) 20.6$

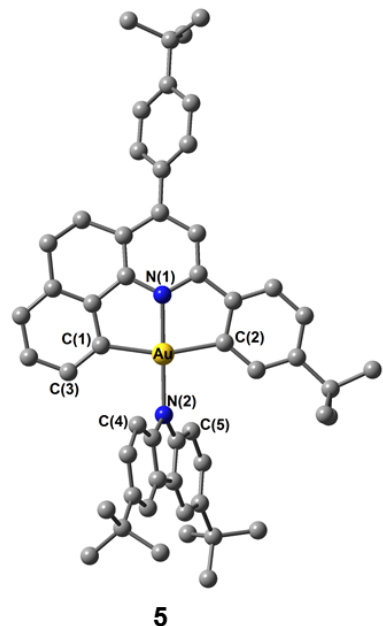

$\mathrm{Au}-\mathrm{C}(1) 2.086 \mathrm{C}(1)-\mathrm{Au}-\mathrm{N}(1) \quad 81.6$

$\mathrm{Au}-\mathrm{C}(2) 2.078 \mathrm{C}(2)-\mathrm{Au}-\mathrm{N}(1) \quad 80.3$

$\mathrm{Au}-\mathrm{N}(1) 1.965 \mathrm{~N}(1)-\mathrm{Au}-\mathrm{N}(2) 176.2$

$\mathrm{Au}-\mathrm{N}(2) \quad 2.023 \mathrm{C}(1)-\mathrm{Au}-\mathrm{N}(2)-\mathrm{C}(4) \quad 73.7$

Figure S19 The $\mathrm{T}_{1}$ geometries and selected structural parameters of 1, 2 and $\mathbf{5}$ optimized at the PBE0 level of theory.

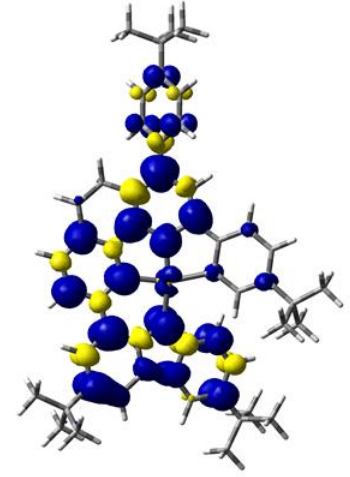

1

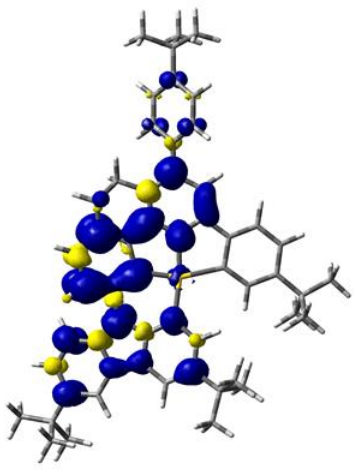

2

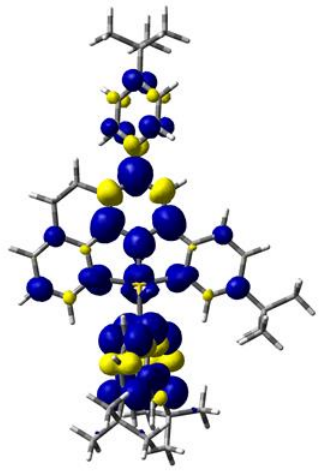

5

Figure S20 Plots of spin density (isovalue $=0.002$ ) of the $T_{1}$ states of $\mathbf{1}, \mathbf{2}$ and $\mathbf{5}$. 


\section{OLED Performance}

Table S16 Key parameters of solution-processable devices made with 1-4.

\begin{tabular}{|c|c|c|c|c|c|}
\hline Complex & $\begin{array}{c}\text { Dopant } \\
\text { Concentration / wt } \%\end{array}$ & $\begin{array}{c}\text { Max. CE / } \\
\text { cd A }^{-1}\end{array}$ & $\begin{array}{l}\text { Max. PE / } \\
\operatorname{lm~W}^{-1}\end{array}$ & $\begin{array}{c}\text { Max. } \\
\mathrm{EQE} / \%\end{array}$ & $\operatorname{CIE}(\mathrm{x}, \mathrm{y})^{[\mathrm{a}]}$ \\
\hline \multirow[t]{4}{*}{1} & 5 & 1.3 & 0.3 & 0.5 & $(0.50,0.49)$ \\
\hline & 10 & 5.5 & 2.1 & 2.2 & $(0.51,0.48)$ \\
\hline & 15 & 6.4 & 3.9 & 2.6 & $(0.51,0.48)$ \\
\hline & 20 & 6.9 & 4.9 & 3.0 & $(0.53,0.47)$ \\
\hline \multirow[t]{4}{*}{2} & 5 & 11.3 & 5.5 & 3.5 & $(0.28,0.57)$ \\
\hline & 10 & 16.5 & 8.2 & 5.2 & $(0.29,0.57)$ \\
\hline & 15 & 21.1 & 11.5 & 6.6 & $(0.30,0.58)$ \\
\hline & 20 & 19.3 & 12.1 & 6.1 & $(0.30,0.58)$ \\
\hline \multirow[t]{4}{*}{3} & 5 & 0.9 & 0.3 & 0.3 & $(0.48,0.51)$ \\
\hline & 10 & 1.2 & 0.5 & 0.5 & $(0.51,0.48)$ \\
\hline & 15 & 2.4 & 1.5 & 1.0 & $(0.52,0.48)$ \\
\hline & 20 & 2.2 & 1.6 & 1.0 & $(0.53,0.47)$ \\
\hline \multirow[t]{4}{*}{4} & 5 & 2.3 & 0.7 & 0.8 & $(0.49,0.50)$ \\
\hline & 10 & 2.5 & 0.9 & 0.8 & $(0.49,0.51)$ \\
\hline & 15 & 4.1 & 2.2 & 1.6 & $(0.50,0.50)$ \\
\hline & 20 & 4.2 & 3.0 & 1.7 & $(0.51,0.49)$ \\
\hline
\end{tabular}

${ }^{\text {[a] }} \mathrm{CIE}$ coordinates are taken at a current density of $10 \mathrm{~mA} \mathrm{~cm} \mathrm{~cm}^{-2}$.

${ }^{[b]} \mathrm{CE}, \mathrm{PE}$, and $\mathrm{EQE}$ denote the current efficiency, power efficiency and external quantum efficiency, respectively. 


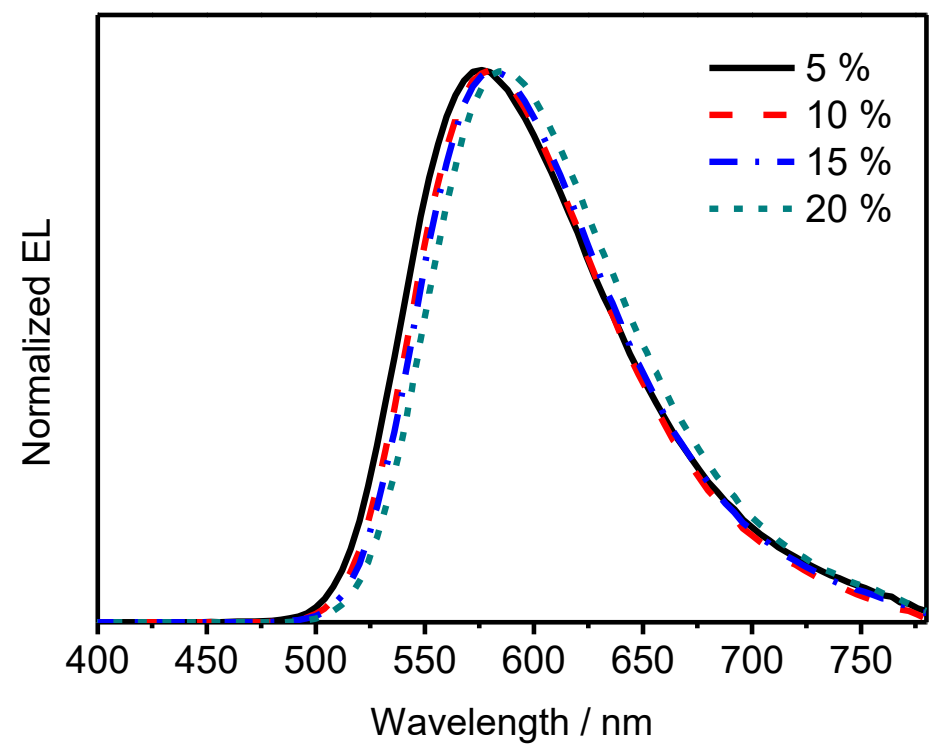

Figure S21 EL spectra of solution-processed devices made with $\mathbf{1}$ at various dopant concentrations. 

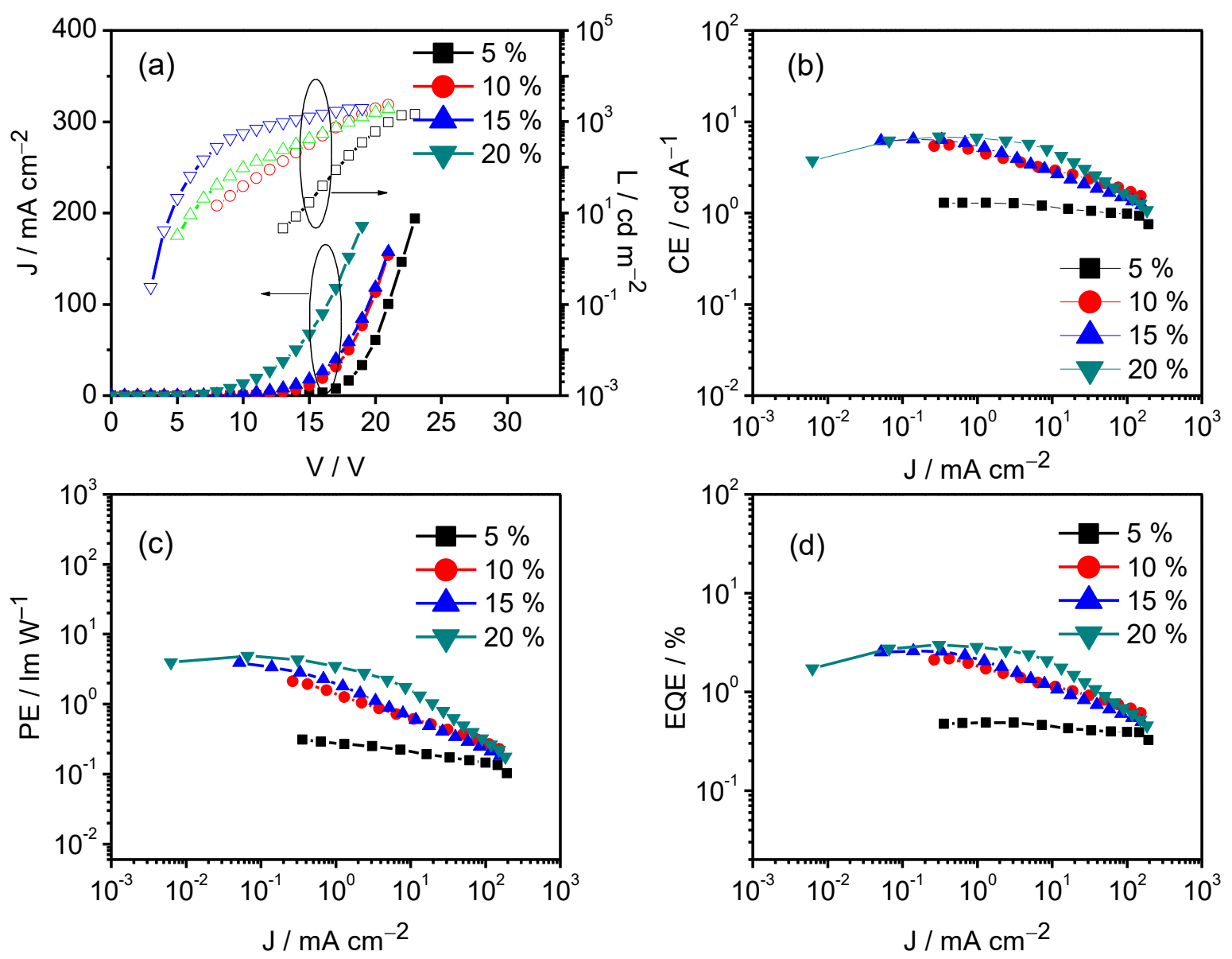

Figure S22 (a) J-V-L characteristics, (b) $\mathrm{CE}$, (c) $\mathrm{PE}$ and (d) EQE as a function of current density of devices doped with $\mathbf{1}$. 

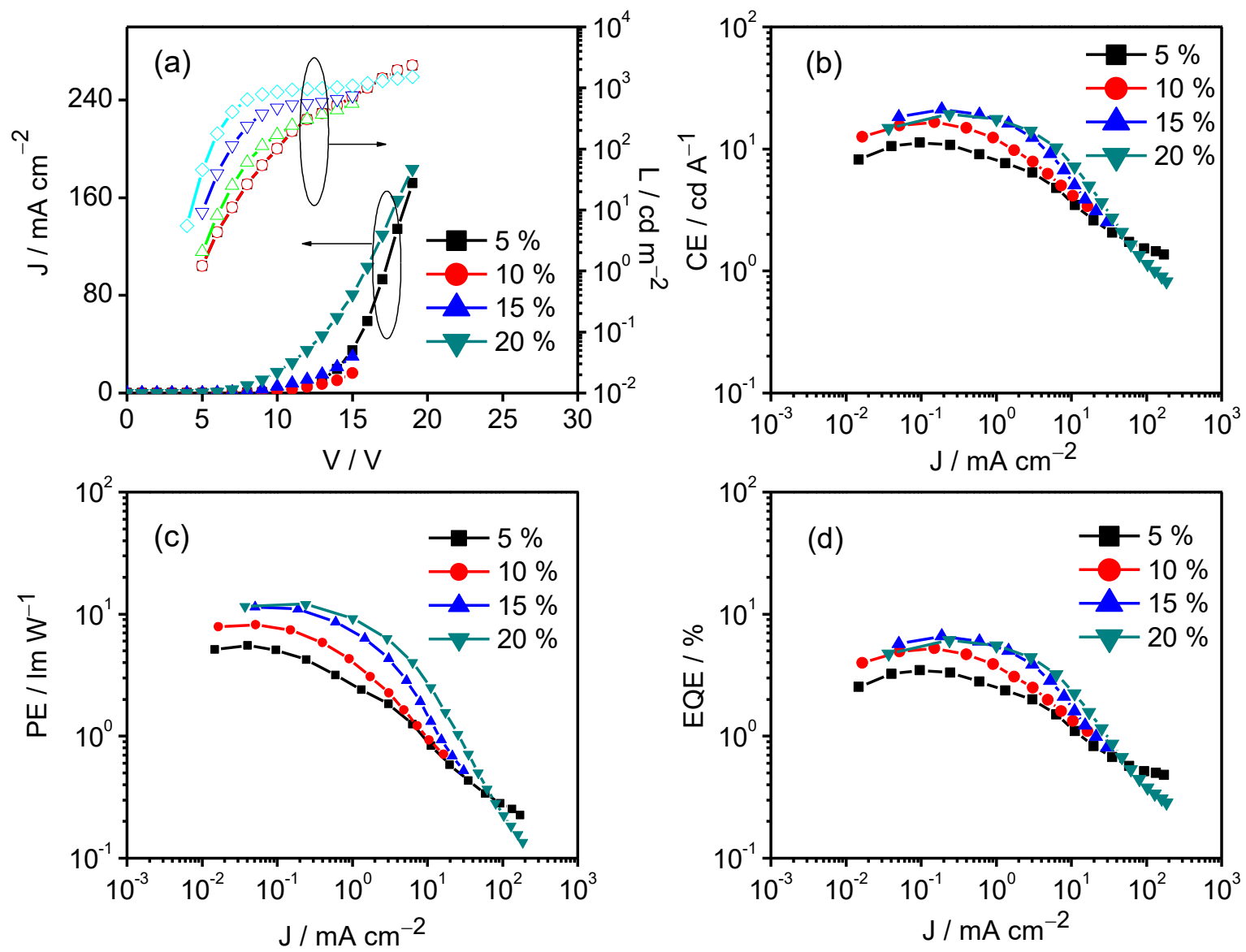

Figure S23 (a) J-V-L characteristics, (b) CE, (c) PE and (d) EQE as a function of current density of devices doped with 2 . 


\section{References}

1 (a) Hegedus, L. S., Organopalladium chemistry. In Organometallics in synthesis: A manual, 2nd ed.; Schlosser, M., Ed. John Wiley \& Sons, Inc.: 2013; pp 1123; (b) Nataro, C.; Fosbenner, S. M. Synthesis and characterization of transition-metal complexes containing 1,1'-bis(diphenylphosphino)ferrocene. J. Chem. Educ. 2009, 86, 1412; (c) Organ, M. G.; Chass, G. A.; Fang, D.-C.; Hopkinson, A. C.; Valente, C. Pd-NHC (PEPPSI) Complexes: Synthetic utility and computational studies into their reactivity. Synthesis 2008, 2008, 2776.

2 Kröhnke, F. The specific synthesis of pyridines and oligopyridines. Synthesis 1976, 1976, 1.

3 Hao, Q.; Yu, S.; Li, S.; Chen, J.; Zeng, Y.; Yu, T.; Yang, G.; Li, Y. Locked planarity: a strategy for tailoring ladder-type $\pi$-conjugated anilido-pyridine boron difluorides. J. Org. Chem. 2014, 79, 459.

4 Crosby, G. A.; Demas, J. N. Measurement of photoluminescence quantum yields. Review. J. Phys. Chem. 1971, 75, 991.

5 Minor, W.; Cymborowski, M.; Otwinowski, Z.; Chruszcz, M. HKL-3000: the integration of data reduction and structure solution - from diffraction images to an initial model in minutes. Acta Crystallogr. D 2006, 62, 859.

6 Sheldrick, G. SHELXT - Integrated space-group and crystal-structure determination. Acta Crystallogr. A 2015, 71, 3.

7 Sheldrick, G. Crystal structure refinement with SHELXL. Acta Crystallogr. C 2015, 71, 3.

8 Fors, B. P.; Krattiger, P.; Strieter, E.; Buchwald, S. L. Water-mediated catalyst preactivation: An efficient protocol for $\mathrm{C}-\mathrm{N}$ cross-coupling reactions. Org. Lett. 2008, 10, 3505.

9 (a) Bauernschmitt, R.; Ahlrichs, R. Treatment of electronic excitations within the adiabatic approximation of time dependent density functional theory. Chem. Phys. Lett. 1996, 256, 454; (b) Casida, M. E.; Jamorski, C.; Casida, K. C.; Salahub, D. R. Molecular excitation 
energies to high-lying bound states from time-dependent densityfunctional response theory: characterization and correction of the timedependent local density approximation ionization threshold. J. Chem. Phys. 1998, 108, 4439; (c) Stratmann, R. E.; Scuseria, G. E.; Frisch, M. J. An efficient implementation of time-dependent density-functional theory for the calculation of excitation energies of large molecules. J. Chem. Phys. 1998, 109, 8218.

10 Frisch, M. J.; Trucks, G. W.; Schlegel, H. B.; Scuseria, G. E.; Robb, M. A.; Cheeseman, J. R.; Scalmani, G.; Barone, V.; Petersson, G. A.; Nakatsuji, H.; Li, X.; Caricato, M.; Marenich, A. V.; Bloino, J.; Janesko, B. G.; Gomperts, R.; Mennucci, B.; Hratchian, H. P.; Ortiz, J. V.; Izmaylov, A. F.; Sonnenberg, J. L.; Williams; Ding, F.; Lipparini, F.; Egidi, F.; Goings, J.; Peng, B.; Petrone, A.; Henderson, T.; Ranasinghe, D.; Zakrzewski, V. G.; Gao, J.; Rega, N.; Zheng, G.; Liang, W.; Hada, M.; Ehara, M.; Toyota, K.; Fukuda, R.; Hasegawa, J.; Ishida, M.; Nakajima, T.; Honda, Y.; Kitao, O.; Nakai, H.; Vreven, T.; Throssell, K.; Montgomery Jr., J. A.; Peralta, J. E.; Ogliaro, F.; Bearpark, M. J.; Heyd, J. J.; Brothers, E. N.; Kudin, K. N.; Staroverov, V. N.; Keith, T. A.; Kobayashi, R.; Normand, J.; Raghavachari, K.; Rendell, A. P.; Burant, J. C.; Iyengar, S. S.; Tomasi, J.; Cossi, M.; Millam, J. M.; Klene, M.; Adamo, C.; Cammi, R.; Ochterski, J. W.; Martin, R. L.; Morokuma, K.; Farkas, O.; Foresman, J. B.; Fox, D. J. Gaussian 09 (Revision D.01), Wallingford, CT, 2013.

11 (a) Adamo, C.; Barone, V. Toward reliable density functional methods without adjustable parameters: the PBE0 model. J. Chem. Phys. 1999, 110, 6158; (b) Perdew, J. P.; Burke, K.; Ernzerhof, M. Generalized gradient approximation made simple. Phys. Rev. Lett. 1996, 77, 3865; (c) Perdew, J. P.; Burke, K.; Ernzerhof, M. Generalized gradient approximation made simple [Phys. Rev. Lett. 77, 3865 (1996)]. Phys. Rev. Lett. 1997, 78, 1396.

12 (a) Cossi, M.; Rega, N.; Scalmani, G.; Barone, V. Energies, structures, and electronic properties of molecules in solution with the C-PCM solvation model. J. Comput. Chem. 2003, 24, 669; (b) Barone, V.; Cossi, M. Quantum calculation of molecular energies and energy gradients in solution by a conductor solvent model. J. Phys. Chem. A 1998, 102, 1995. 
13 Andrae, D.; Häußermann, U.; Dolg, M.; Stoll, H.; Preuß, H. Energy-adjusted ab initio pseudopotentials for the second and third row transition elements. Theoretica chimica acta 1990, 77, 123.

14 Ehlers, A. W.; Böhme, M.; Dapprich, S.; Gobbi, A.; Höllwarth, A.; Jonas, V.; Köhler, K. F.; Stegmann, R.; Veldkamp, A.; Frenking, G. A set of f-polarization functions for pseudopotential basis sets of the transition metals $\mathrm{Sc}-\mathrm{Cu}, \mathrm{Y}-\mathrm{Ag}$ and $\mathrm{La}-\mathrm{Au}$. Chem. Phys. Lett. 1993, 208, 111.

15 (a) Francl, M. M.; Pietro, W. J.; Hehre, W. J.; Binkley, J. S.; Gordon, M. S.; DeFrees, D. J.; Pople, J. A. Self-consistent molecular orbital methods. XXIII. A polarization-type basis set for second-row elements. J. Chem. Phys. 1982, 77, 3654; (b) Hehre, W. J.; Ditchfield, R.; Pople, J. A. Self-consistent molecular orbital methods. XII. Further extensions of Gaussian-type basis sets for use in molecular orbital studies of organic molecules. J. Chem. Phys. 1972, 56, 2257; (c) Hariharan, P. C.; Pople, J. A. The influence of polarization functions on molecular orbital hydrogenation energies. Theoretica chimica acta 1973, 28, 213. 